\title{
Nuclear car wash status report, August 2005
}

S. Prussin, D. Slaughter, J. Pruet, M. Descalle, A. Bernstein, J. Hall, M. Accatino, O. Alford, S. Asztalos, J. Church, A. Loshak, N. Madden, D. Manatt, T. Moore, E. Norman, D. Petersen

August 16, 2005 
This document was prepared as an account of work sponsored by an agency of the United States Government. Neither the United States Government nor the University of California nor any of their employees, makes any warranty, express or implied, or assumes any legal liability or responsibility for the accuracy, completeness, or usefulness of any information, apparatus, product, or process disclosed, or represents that its use would not infringe privately owned rights. Reference herein to any specific commercial product, process, or service by trade name, trademark, manufacturer, or otherwise, does not necessarily constitute or imply its endorsement, recommendation, or favoring by the United States Government or the University of California. The views and opinions of authors expressed herein do not necessarily state or reflect those of the United States Government or the University of California, and shall not be used for advertising or product endorsement purposes.

This work was performed under the auspices of the U.S. Department of Energy by University of California, Lawrence Livermore National Laboratory under Contract W-7405-Eng-48. 


\section{Table of contents}

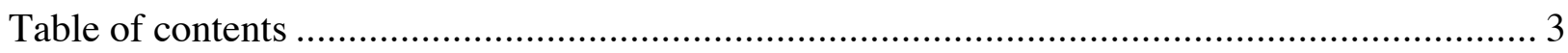

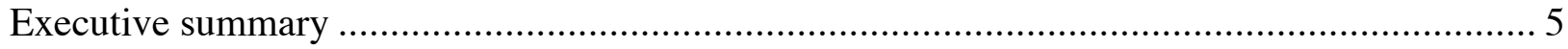

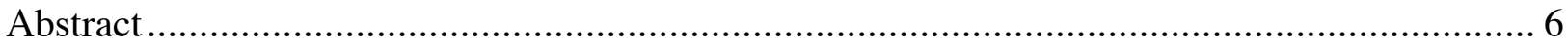

1. Background and motivation ................................................................................ 10

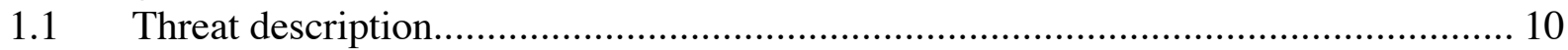

1.2 Conventional neutron interrogation to detect SNM ................................................. 13

1.3 A new signature: Delayed high-energy Grays.................................................... 14

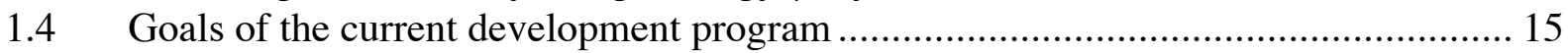

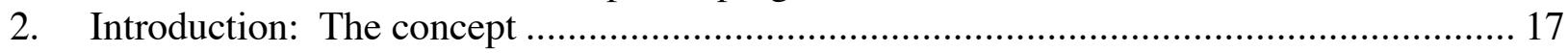

2.1 Enhancement of the Monte Carlo simulation code COG .......................................... 18

2.2 Construction of a laboratory for component testing and code validation ................... 20

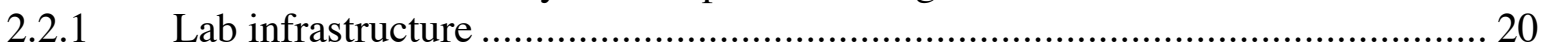

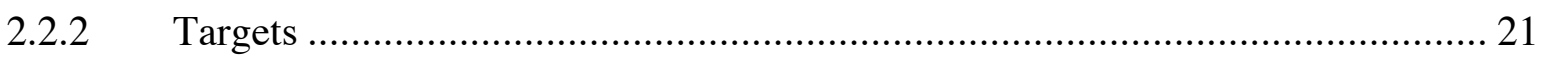

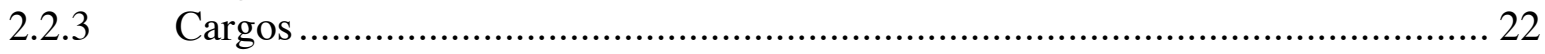

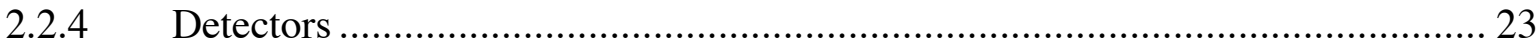

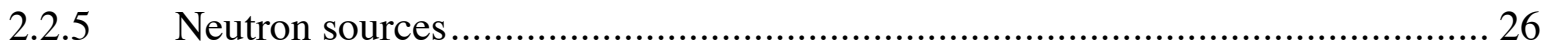

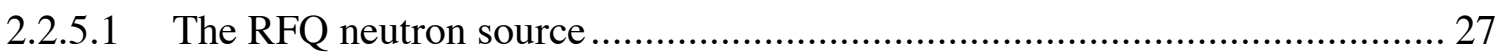

2.2.5.2 RFQ generator installation ..................................................................... 29

3. Qualification of a Computational Model utilizing a $14 \mathrm{MeV}$ neutron source...................... 31

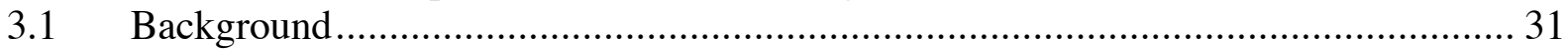

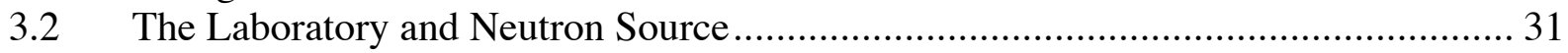

3.2.1 Calibration of the dePangher Long Counter ................................................... 33

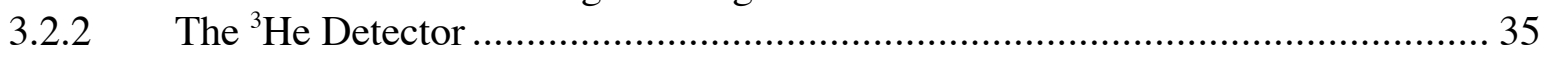

3.2.3 The Beam Monitor of the Generator Output...................................................... 36

3.2.4 Reduction of personnel dose rates............................................................. 37

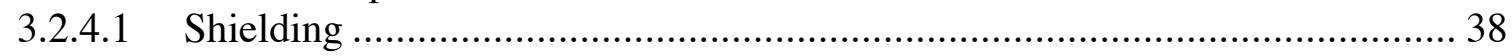

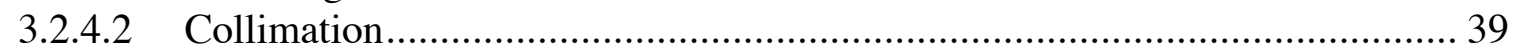

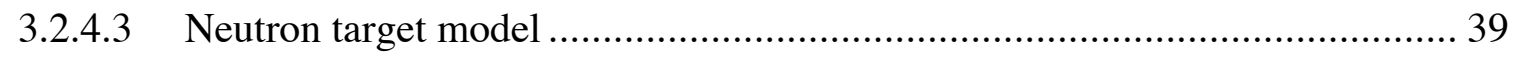

3.2.5 Final assembly of the component testing lab .............................................. 41

3.2.5.1 Cargo translation system............................................................................ 41

3.3 Comparing Long Counter and ${ }^{3} \mathrm{He}$ tube data with the Baseline Model Monte Carlo

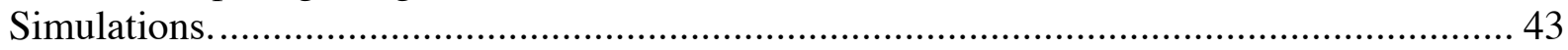

3.3.1 Relative Axial Variation of the Fast Flux due to $14 \mathrm{MeV}$ source...................... 44

3.3.2 Relative Axial Variation of the Low-energy Neutron Flux ................................ 46

3.3.3 Relative Horizontal Variation of the Flux of Low-energy Neutrons .................. 47

3.3.4 Absolute Calibration of the Generator output................................................. 48

3.4 Comparison of the Baseline Model with the Number of Fissions Induced in a HEU

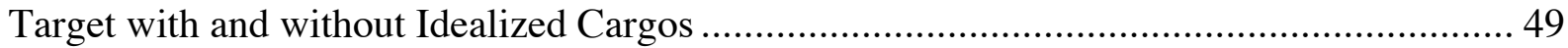

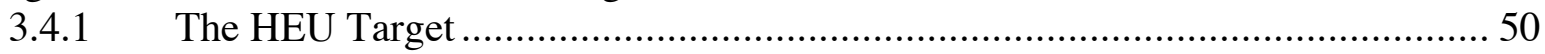

3.4.2 Actual Irradiation Periods ........................................................................ 51

3.4.3 Average Fission Yields of ${ }^{91.92} \mathrm{Sr}$ used to determine fission rate ....................... 52

3.4.4 Full-energy Efficiency of the HPGe Detector.................................................. 52

3.4.5 Monte Carlo Simulation of the Efficiency Function for the HPGe Detector and

Normalization with Experimental Measurements ......................................................... 54

3.4.6 Correction for Attenuation by Tantalum ......................................................... 56 


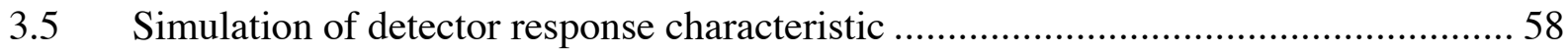

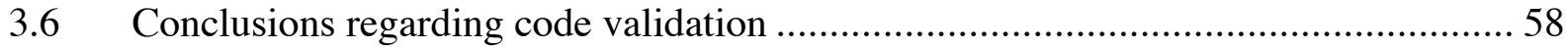

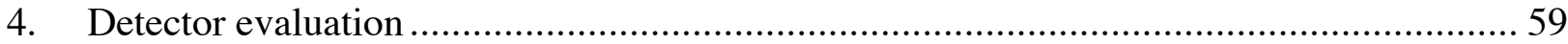

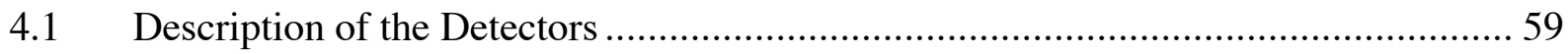

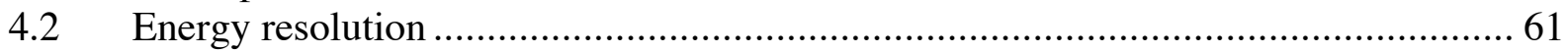

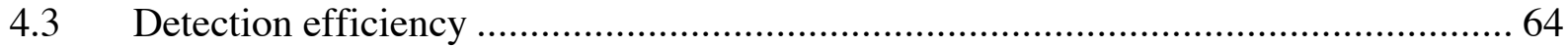

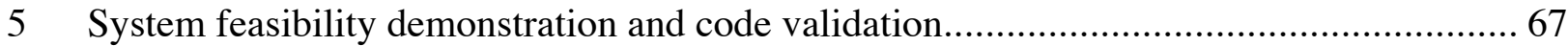

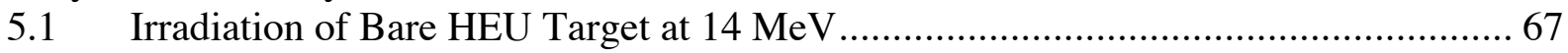

5.2 Irradiation at $14 \mathrm{MeV}$ of HEU Target Embedded in a Steel-pipe Array ..................... 69

5.3 Irradiation at $14 \mathrm{MeV}$ of HEU Target Embedded in Plywood ................................... 71

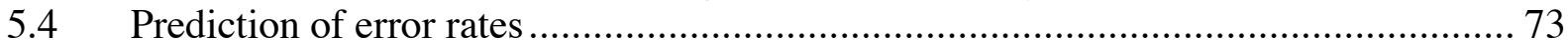

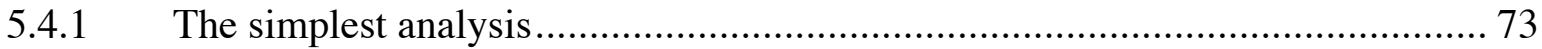

5.4.2 The ROC curve and the 5xSIGMA rule ……................................................ 75

5.4.3 What we do and don't know about the count rate distribution functions ............. 77

5.5 What dose might be required for reliable scanning? ?............................................. 79

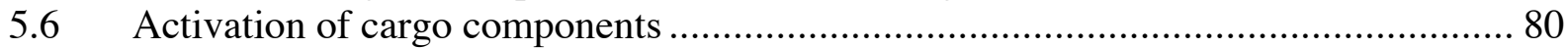

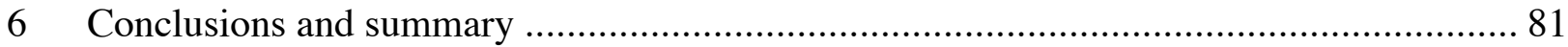

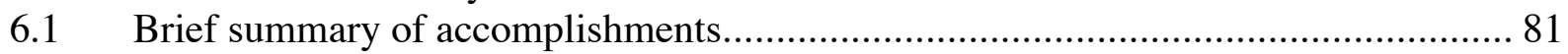

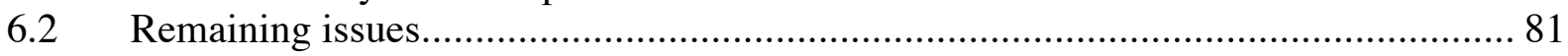

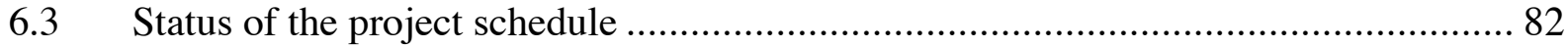

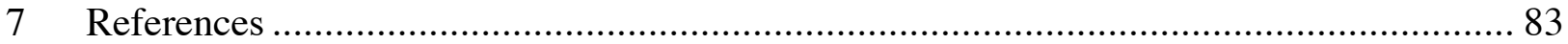




\section{Executive summary}

The goals of the Nuclear Car Wash project are:

a) Develop a concept for screening Intermodal Cargo Containers (IMCC) to detect the presence of SNM

b) Develop simulation tools needed to predict its performance and optimize the engineering design

c) Experimentally validate the simulation tools

d) Test system components in the laboratory with realistic configurations that simulate the seaport environment at full scale.

Many important milestones have been reached in the pursuit of the above goals. Specifically:

- A new concept for neutron interrogation to detect SNM in IMCC has been developed (Section 1.3 and 2.0)

- A heretofore untried radiation signature has been proposed, evaluated, and demonstrated experimentally (Section 1.3, 5.1 through 5.3). A measure of the progress in overall system performance is described in Section 5.

- The Monte Carlo simulation code COG has undergone a major upgrade to include generation of individual fission products at the appropriate yield and to generate [-delayed Gradiation based on individual transitions derived from their known level schemes (Section 2.1). This allows detailed incorporation of the actual nuclear data into predictions of system performance and its optimization

- The upgraded COG code has been experimentally validated (Section 3)

- A new cargo scanning laboratory has been created at LLNL (see Section 2.2) where appropriate samples of HEU can be embedded in real cargo materials at full scale and scanned past an appropriate neutron generator to test components that will become part of an engineered scanning system

- A variety of detectors have been deployed and evaluated experimentally to derive an optimum design (Section 4)

- False positive and false negative error rates have been predicted using a primitive but defensible analysis (Section 5.4) and the results indicate that goals on detection probability and false alarms can be met with this concept in a realistic configuration

- Requirements for an optimum neutron source have been developed (Section 5.4) 


\begin{abstract}
A large majority of US imports arrive at seaports in maritime cargo containers. The number of containers arriving is nearly 10 million per year, each with a cargo of up to 30 tons of various materials. This provides a vulnerable entry point for the importation of a nuclear weapon or its components by a terrorist group. Passive radiation sensors are being deployed at portals to detect radioactive material and portable instruments are carried by port personnel to augment detection. Those instruments can detect the neutrons and Grays produced by ${ }^{240} \mathrm{Pu}$ that is normally present in weapons grade plutonium in cases where cargo overburden is not too great. However, ${ }^{235} \mathrm{U}$ produces almost no neutron output in its normal radioactive decay and its principal $\square$-radiation is at $186 \mathrm{keV}$ and is readily attenuated by small amounts of wood or packing materials. Impurities such as ${ }^{232} \mathrm{U}$, often present in reactor irradiated material at the 100-200 ppt level, can provide a detectable signal through significant cargo overburden but the wide variations among samples of HEU make this an unreliable means of detecting SNM. High quality radiography may be useful in determining that the majority of containers are clearly free of SNM. However, some containers will lead to ambiguous results from radiography and passive radiation sensing.
\end{abstract}

For these reasons active neutron interrogation is proposed as a means to produce fission and thus greatly amplify the radiation output of fissionable material to facilitate its reliable detection even when well shielded by large cargo overburden. Historically, the fission signature utilized as the unique identifying feature of fissionable materials is the detection of delayed neutrons. However, these neutrons have very low yield 0.017 per fission in ${ }^{235} \mathrm{U}$, and their low energy results in very poor penetration of hydrogenous materials such as fuels, water, wood, or agricultural products. That signature alone does not provide reliable detection in thick cargos.

A new signature has been identified and has been developed within the current project for the detection of well shielded SNM. This SNM signature is based on high-energy delayed 7 -radiation produced by fission products following neutron or photon induced fission. These Grays are high enough in energy $\left(\mathrm{E}_{0}>3 \mathrm{MeV}\right)$ to be readily distinguished from any natural background radioactivity since the latter does not extend above $2.6 \mathrm{MeV}$. Their abundance is nearly a decade greater than delayed neutrons and their short half-lives deliver nearly all of the signature radiation on time scales of one minute or less and thus facilitate rapid scanning. Finally, for this F-radiation in the 3-6 MeV range attenuation occurs only by Compton scattering and is in the range where minimum attenuation occurs in all materials. Even the thickest cargos of any material attenuate these - rrays by only a factor of 10-30X so that the signature is readily detected even with the most challenging shield materials.

The goals of the current program are to detect significant quantities (much less than IAEA "significant" amounts) of well-shielded SNM, and to do so with detection probability $P_{d} \geq 95 \%$ and with false alarm rates $P_{\text {fp }} \leq 0.001$. It is the goal to meet these requirements in a scan that requires less than one minute to complete and does so without damage to the cargo or to people who may be hidden inside. We intend to 
meet these requirements even when the cargo overburden is up to $\square \mathrm{L} \leq 150 \mathrm{~g} / \mathrm{cm}^{2}$ of any material ranging from fuels and agricultural products to steel and lead.

Producing a field prototype requires accurate, validated, and reliable simulation tools. At the beginning of this effort no simulation tools were available that generate and track individual fission product nuclides and their subsequent high-energy Gray emission. The LLNL transport code COG was enhanced with a physics package and a nuclear database of fission product data to simulate the production of fission products and their emission following - -decay, as described in Section 2.1. This code was modified to run on a cluster of desktop computers to provide rapid turn around of simulations. It has been validated by comparison to experimental measurements made with real HEU in real cargos in a container with a neutron generator set at realistic distance from the cargo. Results show good agreement between predictions and experimental results. The code has also been enhanced to simulate the response of various detectors to the high-energy Gray spectrum so that simulations are now capable of predicting the actual pulse height spectrum obtained during a measurement, and thus the actual count rate expected in a field prototype.

A cargo-scanning laboratory has been created where experimental mock ups of real cargo scanning configurations can be tested and results compared to recently developed simulation tools as explained above. This is described in Sections 2, 3 and 5. The lab has available targets of $90-97 \%$ enriched ${ }^{235} \mathrm{U}$ at masses of $50-400 \mathrm{~g}$. It is permitted to field targets up to $800 \mathrm{~g}{ }^{235} \mathrm{U}$ and for that we deploy several smaller targets simultaneously. Shielding has been designed to allow safe occupancy of a control console and surrounding offices for people not connected with this effort. Routine interrogations produce radiation dose rates too low to be recorded on personnel dosimeters even for those operating the neutron source. An assortment of cargo materials has been assembled on pallets including wood and steel cargos at full scale and at mean density $\square \sim 0.5-0.6 \mathrm{~g} / \mathrm{cm}^{3}$ characteristic of a fully loaded contained at its weight limit. Wood is chosen because its radiation transport properties are a good surrogate for fuels, agricultural products, and packing materials. A diverse array of detectors has been installed for evaluation and ultimate choice of particular designs based on operational experience. The detectors include large liquid scintillation detectors and a variety of solid plastic detectors. The plastic detectors were made in 29$61 \mathrm{~cm}$ dimensions, $61 \times 61 \mathrm{~cm}$ and $61 \times 122 \mathrm{~cm}$ in thickness of $13 \mathrm{~cm}$ and $25 \mathrm{~cm}$. Some had a single photomultiplier while others had two on the ends or on the side. These are described in Section 4.

A $14 \mathrm{MeV}$ neutron generator was installed and used to successfully detect the SNM targets buried in $\square \mathrm{L} \leq 60 \mathrm{~g} / \mathrm{cm}^{2}$ of cargo overburden as described in Sections 5.1-5.3. It's output due to the $\mathrm{T}(\mathrm{d}, \mathrm{n})$ reaction is isotropic and produces $\square \sim 8 \times 10^{4} \mathrm{n} / \mathrm{cm}^{2} / \mathrm{s}$ at $\mathrm{R}=2.5 \mathrm{~m}$ without attenuation. A new neutron generator has been designed, procured, and installed to produce 3-7 MeV neutrons with intensity $\square \sim 1 \times 10^{6} \mathrm{n} / \mathrm{cm}^{2} / \mathrm{s}$ at $\mathrm{R}=2.5$ $\mathrm{m}$. It's output is due to the $\mathrm{D}(\mathrm{d}, \mathrm{n})$ reaction produced by a $4 \mathrm{MeV}$ deuteron beam incident on a $\mathrm{D}_{2}$ gas target. This reaction produces a beam highly peaked in the forward direction so that, although the intensity at the target is much higher than the D-T generator produced, the total output is comparable to the earlier source and thus activation of nearby components and dose to operating personnel is similar to or less 
than with the earlier unit. More importantly, the reduced neutron energy eliminates oxygen activation due to the ${ }^{16} \mathrm{O}(\mathrm{n}, \mathrm{p})^{16} \mathrm{~N}$ reaction whose neutron energy threshold is $10 \mathrm{MeV}$. The latter reaction produced significant interference when the ${ }^{16} \mathrm{~N}$ decay with its $7.1 \mathrm{~s}$ half-life and $6 \mathrm{MeV}$-ray principal emission masked a good deal of the fission product signature.

The laboratory described above was outfitted with a full set of diagnostic instruments so that absolute intensities could be determined for comparison of experimental measurements with detailed simulations. A calibrated precision long counter was utilized to establish the absolute neutron generator output with high accuracy, and neutron beam monitors were installed to monitor fluctuations during a run and between runs. Several ${ }^{3}$ He proportional counters, with and without moderation, were employed to map neutron fields throughout the exposed volume. A large (70\%) HPGe high-resolution Iray spectrometer was calibrated and used to identify activation products, fission products, and interferences. It was also used at the end of a run to make an absolute determination of the number of fissions produced in the target for comparison with simulations.

Measurements made in the lab system described above have been used to determine absolute fluxes and their uncertainties. These have been compared to simulations of the experimental configurations. The enhanced COG code reliably predicts the measured fission rates within $\pm 15 \%$. Subsequent generation and transport of the delayed high-energy -radiation is predicted by COG and the result is in very good agreement with experiment. Prediction of pulse height spectra in the detectors is just now being compared to measurements and the results are in very good agreement so far as the comparison has been carried. Validation of the simulation tools is an essential milestone and now its completion facilitates detailed engineering design and optimization of field systems that will be developed and manufactured by our industrial partner.

Currently the lab is being outfitted with a translation system so that a fully loaded $20 \mathrm{ft}$ cargo container can be scanned over the neutron source. This allows the study of background variations that could introduce false alarms, the study of voids as they contribute to signature fluctuations as the container is scanned, and to demonstrate the ability to reliably detect SNM targets on time scales less than one minute.

Measurements scheduled for late summer are expected to demonstrate an even more robust signature with the new $3-7 \mathrm{MeV}$ neutron source when the ${ }^{16} \mathrm{~N}$ interference is eliminated. The features of hard Gray energy spectra and several characteristic fission product decay times are clearly demonstrated in these measurements even when the cargo thickness is large and when it is steel or wood.

Finally, error rates including false positives (false alarms) and false negatives (failure to detect) depend on background count rates, their statistical distribution, and discriminator levels set to detect SNM. Measurements of the background distribution function have been made and utilized to make a primitive prediction of error rates. Of course only operational data can actually provide bona fide error rate determinations. But in the meantime the measured distributions have been utilized to make predictions. 
These data indicate that the error rate goals can be accomplished within the detector size and source intensity parameters currently under study. They also indicate that the goals can be met without undue radiation dose to the cargo contents and thus without damage or reduction in commercial value of the materials being screened. Details of these analyses are given in Section 5.4.

Overall, the following sections describe creation of a new experimental facility for testing cargo-screening components based on neutron interrogation. Appropriate surrogate components are available in this lab. A new computer simulation capability has been developed and validated experimentally in this lab using realistic cargo, source, and detector configurations. Early results indicate that the new SNM signature based on high-energy delayed--rradiation is robust, potentially free of interferences, and reliable even when challenged by the maximum thickness of cargo overburden consisting of worst case materials. All indications at this point suggest that reliable detection of well-shielded SNM can be accomplished without damage to the cargo materials, or undue safety hazards even to people surreptitiously hidden in the cargo container. 


\section{Background and motivation}

\subsection{Threat description}

The majority of cargo entering the US is through the seaports, which receive more than 6 million cargo containers each year. Today approximately $90 \%$ of the world's trade moves by cargo containers[1]. Fortunately this traffic is geographically compact where half of all the traffic bound for the US originates in the top ten foreign ports and nearly $90 \%$ of the cargo containers arrive here at the top ten US ports of entry, as shown in the table below.

Table 1.1 Ports of origin and ports of entry for US-bound cargo containers [1] in 2001.

\begin{tabular}{|c|c|c|c|c|c|}
\hline \multicolumn{3}{|c|}{ Top ten foreign ports or origin } & \multicolumn{3}{|c|}{ Top ten domestic ports } \\
\hline Port of origin & $\begin{array}{l}\text { Number } \\
\text { US-bound } \\
\text { containers }\end{array}$ & $\begin{array}{r}\% \text { of } \\
\text { total } \\
\text { traffic }\end{array}$ & Port of entry & $\begin{array}{l}\text { Number } \\
\text { US-bound } \\
\text { containers }\end{array}$ & $\begin{array}{r}\% \text { of } \\
\text { total } \\
\text { traffic }\end{array}$ \\
\hline Hong Kong & 558,600 & 9.8 & Los Angeles & $1,774,000$ & 24.7 \\
\hline Shanghai & 330,600 & 5.8 & Long Beach & $1,371,000$ & 19.1 \\
\hline Singapore & 330,600 & 5.8 & $\begin{array}{l}\text { New York, } \\
\text { New Jersey }\end{array}$ & $1,044,000$ & 14.6 \\
\hline $\begin{array}{l}\text { Kaohsiung, } \\
\text { Taiwan }\end{array}$ & 319,200 & 5.6 & Charleston & 376,000 & 5.2 \\
\hline Rotterdam & 290,700 & 5.1 & Savannah & 312,000 & 4.3 \\
\hline $\begin{array}{l}\text { Pusan, South } \\
\text { Korea }\end{array}$ & 285,000 & 5.0 & Norfolk & 306,000 & 4.3 \\
\hline Bremerhaven & 256,500 & 4.5 & Seattle & 284,000 & 4.0 \\
\hline Tokyo & 159,600 & 2.8 & Tacoma & 273,000 & 3.8 \\
\hline Genoa & 119,700 & 2.1 & Oakland & 268,000 & 3.7 \\
\hline Yantian, China & 114,000 & 2.0 & Houston & 233,000 & 3.3 \\
\hline Top ten total & $2,764,500$ & 48.5 & $\begin{array}{l}\text { Top ten } \\
\text { total }\end{array}$ & $6,241,000$ & 87.0 \\
\hline
\end{tabular}

For example, the port of Los Angeles/Long Beach is one of the busiest ports in the US and received over three million cargo containers in 2001; approximately half of the total traffic arriving at US ports. One such shipment at the Port of Oakland is shown in the figure below. 


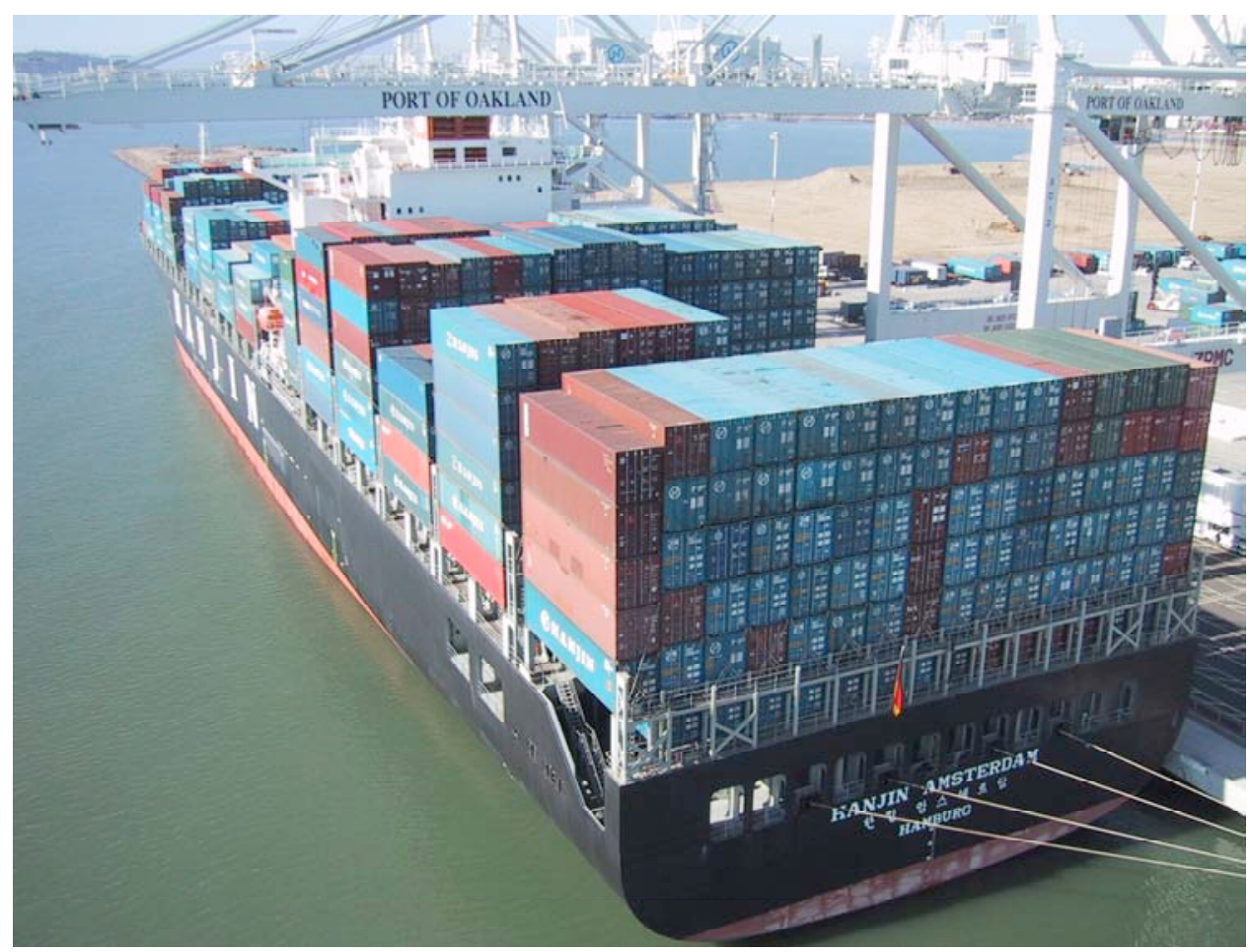

Fig 1.1

A loaded container ship at Port of Oakland

The rate of container arrivals at US ports is expected to increase dramatically over the coming decade. The West Coast ports of Los Angeles/Long Beach, Oakland, and Seattle/Tacoma are currently processing 11,000 containers per day, or 8 per minute on a 24/7 basis. With so many containers and a large volume and mass of cargo in each one it is clear that these containers provide an attractive venue for smuggling illicit material into the US, including weapons of mass destruction (WMD). Because successful delivery of just one such weapon can have catastrophic consequences it is essential that all cargo containers entering the US be screened with an extremely high probability of detecting any WMD hidden within. The cost of failure is very high. An OECD report[2] estimates that a successful WMD attack would shutdown the entire maritime shipping system for a period up to 10 days and this would produce economic costs in the US alone of up to 58 billion dollars.

The Intermodal Cargo Containers (IMCC) in which these cargos arrive are a mixture of $20 \mathrm{ft}$ and $40 \mathrm{ft}$ standard boxes, some with steel sides, others without sides, some with canvas tops, some with tanks for carrying liquids, and some with refrigeration units on one end. The standard boxes[3] are described in Table 1.1-1 below for two versions each of the long and short containers. For the purpose of estimating radiation attenuation in these containers the important parameter is the areal density $\square \mathrm{L}$ where $\square$ is the mean density and $\mathrm{L}$ is the distance from the wall to the container centerline (i.e. half of the width). 
Table 1.1-1 Characteristics of IMCC containers[3]

\begin{tabular}{|c|c|c|c|c|c|c|c|l|}
\hline $\begin{array}{c}\text { Container } \\
\text { description }\end{array}$ & $\begin{array}{c}\text { Length } \\
(\mathrm{cm})\end{array}$ & $\begin{array}{c}\text { Width } \\
(\mathrm{cm})\end{array}$ & $\begin{array}{c}\text { Height } \\
(\mathrm{cm})\end{array}$ & $\begin{array}{c}\text { Weight } \\
\text { limit } \\
(\mathrm{kg})\end{array}$ & $\begin{array}{c}\text { Mean } \\
\text { density at } \\
\text { weight } \\
\text { limit } \\
\left(\mathrm{g} / \mathrm{cm}^{3}\right)\end{array}$ & $\begin{array}{c}\text { Areal } \\
\text { density to } \\
\text { centerline } \\
\square \mathrm{L}\left(\mathrm{g} / \mathrm{cm}^{2}\right)\end{array}$ & $\begin{array}{c}\text { Overload } \\
\text { at weight } \\
\text { limit } \\
\left(\mathrm{g} / \mathrm{cm}^{3}\right)\end{array}$ & $\begin{array}{l}\text { Overload } \\
\text { Areal } \\
\text { density } \square \mathrm{L} \\
\left(\mathrm{g} / \mathrm{cm}^{2}\right)\end{array}$ \\
\hline $20 \mathrm{ft}$ & 593.5 & 237 & 237 & 18,370 & 0.55 & 65 & 0.69 & 81.6 \\
\hline & 589.5 & 239.5 & 239.5 & 21780 & 0.645 & 77.2 & 0.806 & 96.5 \\
\hline $40 \mathrm{ft}$ & 1203.3 & 235.2 & 235.2 & 26700 & 0.401 & 47.2 & 0.501 & 59.0 \\
\hline & 1206.9 & 235.0 & 235.0 & 29650 & 0.444 & 52.3 & 0.556 & 65.3 \\
\hline
\end{tabular}

Examination of the table shows that when these containers are loaded homogeneously at their weight limit the density is in the range $\square=0.4-0.65 \mathrm{~g} / \mathrm{cm}^{3}$ for properly loaded containers and the areal density $\square \mathrm{L}$ is in the range $47-77 \mathrm{~g} / \mathrm{cm}^{2}$. However, these containers are frequently loaded up to $25 \%$ above their weight limit in which case the density may increase to $\square \leq 0.8 \mathrm{~g} / \mathrm{cm}^{3}$ even when loaded homogeneously and the areal density may reach nearly $\square \mathrm{L} \leq 100 \mathrm{~g} / \mathrm{cm}^{2}$. Normal variations encountered with mixed cargos that are not loaded homogeneously can readily increase the areal density up to $\square \mathrm{L} \leq 150 \mathrm{~g} / \mathrm{cm}^{2}$.

The cargo materials are a highly diverse array of textiles, agricultural products, food, fuels, wood, machinery, vehicles, vehicle parts, electronics, and so on. The materials vary seasonally and the proportions of each commodity are distinct to each port of origin and port of entry into the US. Detailed descriptions of manifests and overviews of cargo composition are available from Tarver[4] and have been utilized in developing models described later in this document. Just one snapshot is illustrative. Below is a diagram showing the proportions of various commodities entering the Port of Oakland during one month. 

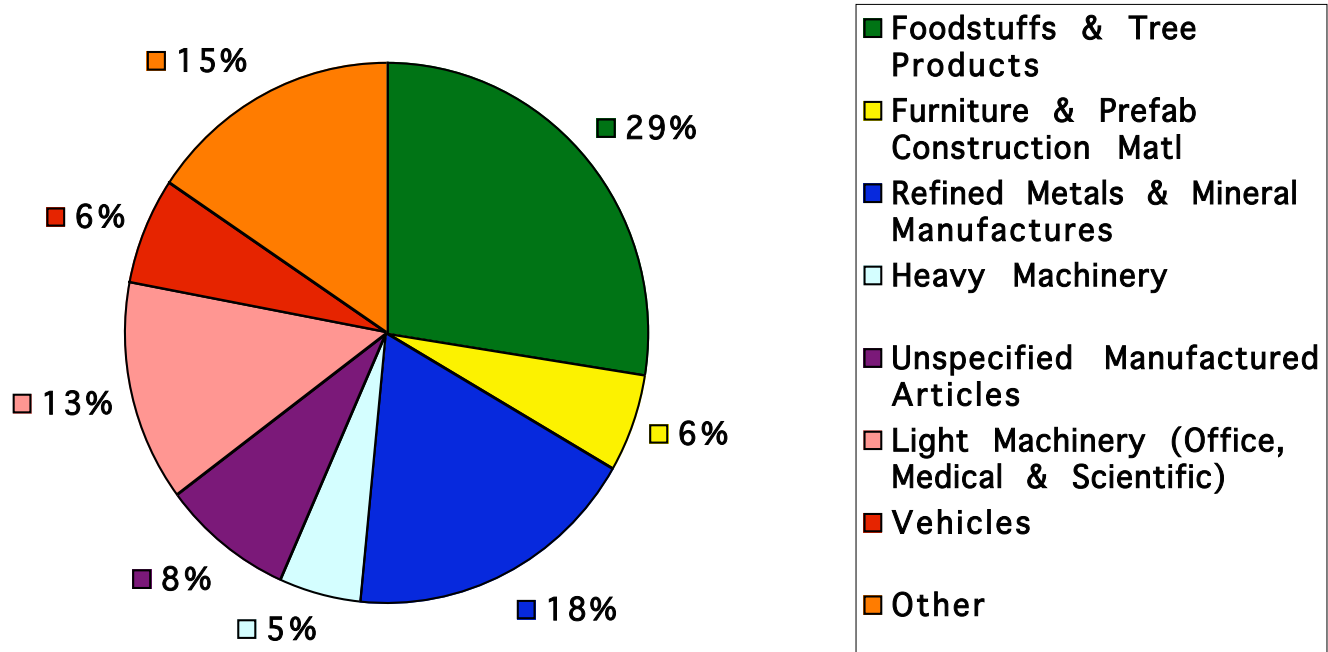

Figure 1.1-1 Proportions of various commodities entering the Port of Oakland.

\subsection{Conventional neutron interrogation to detect SNM}

Among nuclear weapons the ${ }^{239} \mathrm{Pu}$ components have weak but detectable radioactive emissions and they may be detectable using passive nuclear techniques, though detection through thick cargo overburden is not reliable. On the other hand, ${ }^{235} \mathrm{U}$ in the form of "highly enriched uranium" (HEU) components emit only weak, low-energy Grays, with principal emission at $186 \mathrm{keV}$, that are severely attenuated by even small cargo over-burden. The mean free paths at $186 \mathrm{keV}$ in water, aluminum, iron, and lead are only $\square \mathrm{L}=7,8,6$, and $0.8 \mathrm{~g} / \mathrm{cm}^{2}$ respectively. HEU can sometimes be detected passively if previous exposure in a reactor has produced ${ }^{232} \mathrm{U}$ in the sample, or the enrichment is low enough to allow a significant quantity of residual ${ }^{238} \mathrm{U}$ since both these species produce significant $\square$ ray emission at $\mathrm{E}_{5}=1 \mathrm{MeV}$ that can penetrate some cargo. Reliance on detection of these impurity nuclides offers a means of detecting HEU passively whose reliability may be low. This problem is even more difficult because natural radioactive decay of ${ }^{235} \mathrm{U}$ produces negligible neutron emission $\left(\sim^{\prime} 0.006 \mathrm{n} / \mathrm{h}\right.$ per $\left.\mathrm{kg}\right)$, though the impurity nuclides produce $\sim 10 \mathrm{n} / \mathrm{s}$ per $\mathrm{kg}$. For these reasons the concept described below utilizes active neutron interrogation to produce fission where high yields of delayed fission product neutron and Gray emission provides for the reliable identification of fissionable material even when the cargo overburden is thick. A review of current methods for detecting SNM, both passive and active was included in an earlier document of this project and a full bibliography is contained there[5]. 
Work reported in the following sections will focus on detection of shielded HEU since that is a very difficult problem not adequately addressed in the published literature over the past several decades. The technique described here utilizes large, high-efficiency detectors that will also be useful in passively scanning cargo containers and may detect ${ }^{239} \mathrm{Pu}$ by its normal radioactive decay. This work introduces a new signature unique to fission that has not been utilized previously and is sufficiently robust to meet the SNM detection requirements for maritime cargo containers and this will be articulated in the following sections.

\subsection{A new signature: Delayed high-energy Grays}

There are many short-lived fission products that produce abundant $\square$ rays following $\square$-decay (roughly 6-8 Grays per fission) and many have half-lives $\mathrm{T}_{1 / 2}<60 \mathrm{sec}$. Some of the $\square$-decays have very high energies allowing population of highly excited states and copious emission of high-energy $\square$-rays that are distinct from the natural background radiation where high-energy $\square$ rays are almost nonexistent. If we detect only the top $2 \%$, i.e. $\sim 0.13$ Grays per fission, these have energies $\mathrm{E}_{\square}>3 \mathrm{MeV}$. Utilization of this signature for detection of SNM was first proposed recently by Norman and Prussin[6] and it is the primary tool used here to detect well shielded SNM. Details of this signature will be discussed in a later section. Its advantages are:

- Delayed high-energy $\left(\mathrm{E}_{\square}>3 \mathrm{MeV}\right)$ fission product -rays are produced with total intensities approximately $\sim 10$ times larger than that of delayed neutrons[5] in thermal neutron fission of ${ }^{235} \mathrm{U}$ or ${ }^{239} \mathrm{Pu}$.

- High-energy Grays suffer only 10-20X attenuation in thick hydrogenous cargos than is the case for delayed neutrons that suffer $10^{3}-10^{5}$ attenuation.

- High-energy Grays are a distinctive signature of SNM. They are not present in the normal radioactive background that extends only to $\mathrm{E}_{\square} \leq 2.6 \mathrm{MeV}$, and not produced in high abundance by neutron activation products in cargos or cargo environments.

The reduced attenuation of Gradiation in the energy range 3-6 MeV is shown in the figure below. 


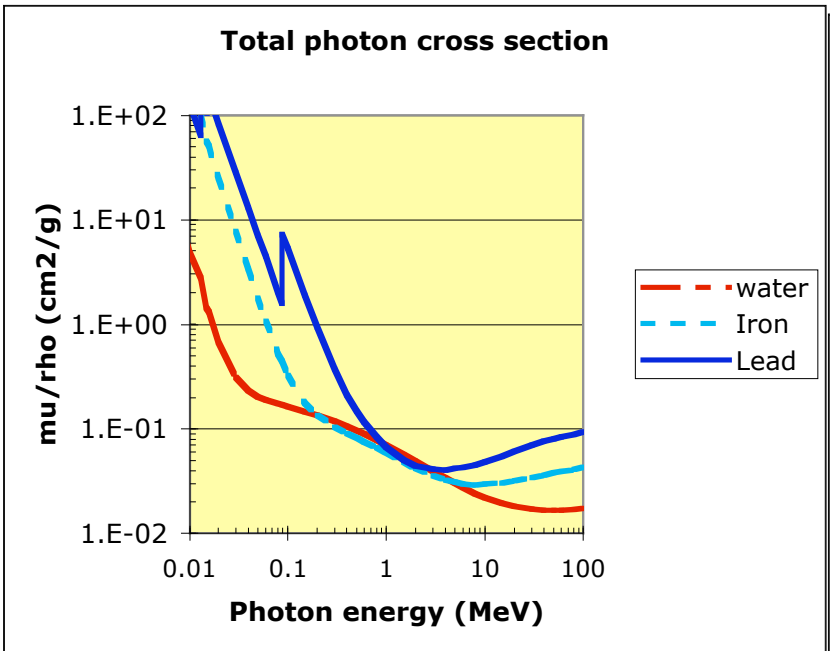

(a)

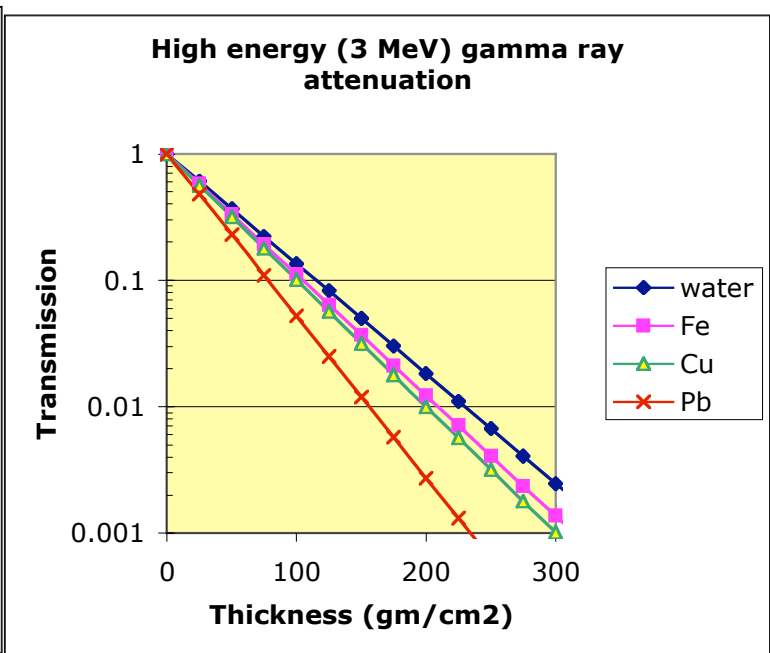

(b)

Figure 1.3-1 Attenuation of [radiation in several important cargo materials and prospective shielding materials. a) Total photon cross section, $\square / \square\left(\mathrm{cm}^{2} / \mathrm{g}\right)$ and b) Transmission in water, iron, copper, and lead.

This signature has not been utilized in earlier reports of neutron or Gray interrogation of cargo. The detector technology for harvesting the substantially larger flux of Grays escaping a thick cargo is well developed and costs are reasonable. The details of possible interferences from background and other sources from a broad range of cargo types have not been developed and are the focus of the work reported below and to be done in the future.

Of course it remains true that neutrons penetrate some cargos better than Gradiation even when the latter is high-energy. An example would be a cargo of steel or machinery or car parts. In that case delayed neutrons emitted by the decay of fission products could, in the case of thick cargo, produce a greater exiting intensity than the high-energy Gradiation. Consequently, a deployed scanning system should observe both delayed neutrons and high-energy Gradiation exiting from a cargo as an indication that SNM is present. The work described below does not address delayed neutrons only because that is considered a simple enhancement of the concept described here.

It is also important to note that the advantages of this new signature accrue for both neutron and Gray interrogation systems. The considerations noted above indicate that some cargos are more readily penetrated by high-energy neutrons than by high-energy Gradiation and in those cases neutron interrogation with this newly developed signature would be the preferred option along with detection of delayed neutrons. In other cases where high-energy $\square$-radiation penetrates those cargos better than neutrons this signature may be the preferred option following photon interrogation of cargo.

\subsection{Goals of the current development program}

The primary goals of the technical work described here are the development of an engineering concept, development of the tools needed to predict and optimize its performance, experimental validation of those tools, and experimental evaluation of system components. 
The goals of the SNM detection are the focus of the technical work are articulated in terms of threat scenarios, costs, and operational constraints. An important element of this analysis is the experience at seaports indicating that the largest part of screening expense is that due to the cost of responding to false alarms. Thus error rates are a central issues. The IAEA defines a "significant amount" of SNM to be comprised of $35 \mathrm{~kg} \mathrm{HEU}$ or $8 \mathrm{~kg} \mathrm{Pu}$.

- The goals of the current program are to detect SNM in amounts nearly a decade smaller than those of concern to the IAEA, i.e. $\sim 1 \mathrm{~kg}$ Pu and $\sim 5 \mathrm{~kg} \mathrm{HEU}$.

- In order to not interfere with commerce at seaports the detection of SNM in a fully loaded cargo container should be completed in about one minute, or less.

- An effective system must have at least a $\mathrm{P}_{\mathrm{d}} \geq 95 \%$ probability of detecting the goal quantities in the time available.

- Responding to false positives is extremely expensive and disruptive to the flow of commerce. The present effort seeks to have no more than $\mathrm{P}_{\mathrm{fp}} \leq 0.001$ false positives per container.

- Finally, the above requirements should be met even when the intervening cargo has areal density up to $\square \mathrm{L} \leq 150 \mathrm{~g} / \mathrm{cm}^{2}$. This is motivated by several observation:

- a homogeneously loaded cargo at the container weight limit has a maximum density (see Section 1.1) in the range $\square=0.4-0.7 \mathrm{~g} / \mathrm{cm} 3$ and the distance from the wall to the container centerline is $\sim 1.3 \mathrm{~m}$ so that the areal density on average may be as large as $100 \mathrm{~g} / \mathrm{cm}^{2}$,

○ port operating staff note that containers are commonly loaded to weights up to 25 "\% larger than the weight limit thus increasing the mean density to $\square " \sim 0.75 \mathrm{~g} / \mathrm{cm}^{3}$ in some containers

- normal variations in cargo packing could nearly double that density in some regions and we set an upper bound $\square \mathrm{L} \leq 150 \mathrm{~g} / \mathrm{cm}^{2}$.

0 this upper bound is also motivated by the observation that a one cubic meter shield box with walls $\square \mathrm{L}=150 \mathrm{~g} / \mathrm{cm}^{2}$ thick would weigh 10 metric tons. A mass that large occupies $1 / 3$ of the container weight limit and larger shield boxes would thus be impractical. 


\section{Introduction: The concept}

Engineering development of a detection system for deployment at seaports requires detailed modeling of radiation transport into and out of a cargo being inspected. Advanced systems will require optimization to maximize reliability and efficacy while reducing cost. Development of the needed design tools and realistic experimental evaluation of candidate system components is essential to the engineering design of a reliable scanning system.

A concept for screening cargo containers to the presence of SNM has been developed and is referred to here as the "nuclear car wash". A pictorial representation of the concept is shown below.

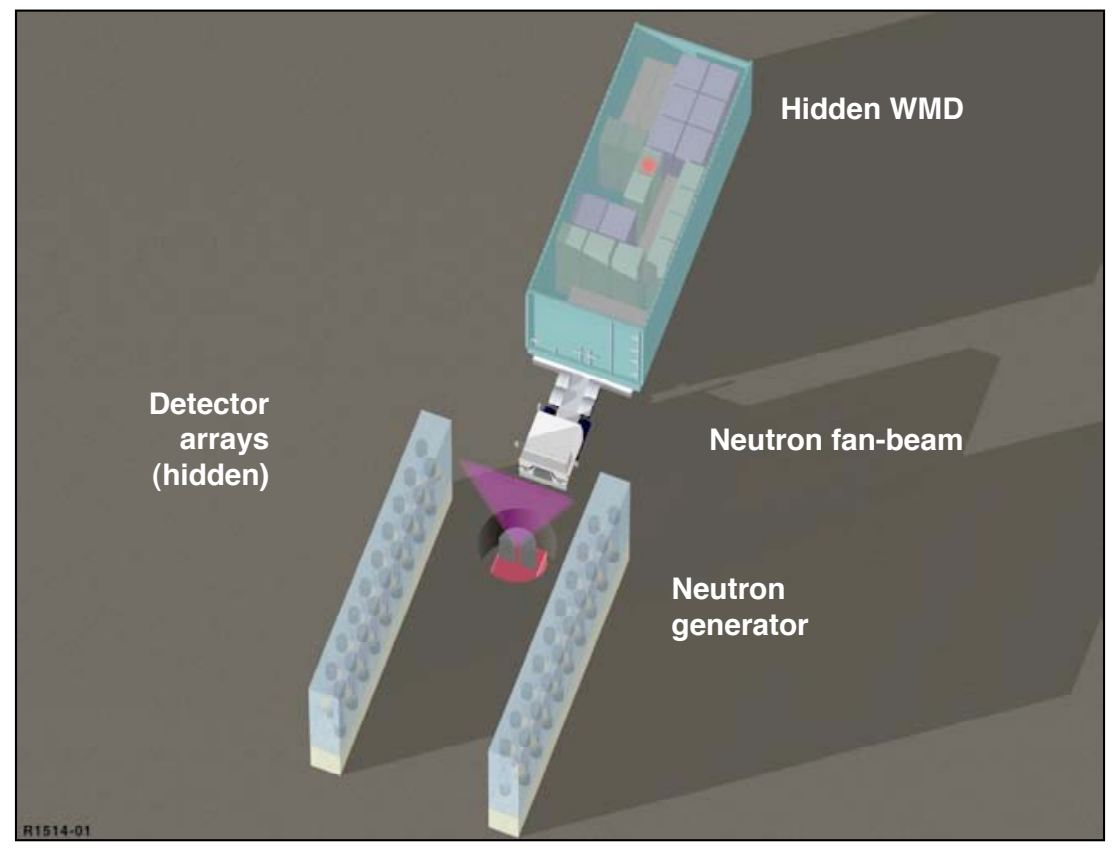

Figure 2-1 The "Nuclear Car Wash” concept.

A standard IMCC cargo container is scanned over a neutron source buried below ground. Alternatively, the container could be towed below a neutron source that was mounted in an overhead cargo container and pointed toward the ground. The source is fan collimated illuminating a slice of the container as it passes the source (either below or above). Should there be SNM in the cargo the neutrons will induce fission and subsequent $\square$-decay of the fission products is detected in two long arrays of detectors as the SNM hot spot moves along the arrays. The detectors consist of very large plastic scintillation detectors whose threshold is set to detect only those fission product $\square$-rays above $\sim 3 \mathrm{MeV}$ and thus they do not respond to normal terrestrial radioactivity. In addition, an array of delayed neutron detectors would be implemented as a thin layer on the inside of the - -detector array so that both of the delayed fission product radiations are monitored in tandem to provide maximum reliability over the full range of cargo materials and configurations. Details of the concept and rationale for the choice of signature and detectors have been presented in several conference and journal papers[7-9]. There are several primary focus areas that define the tasks undertaken in this work. The 
following sections will address each of those tasks and indicate the state of progress, accomplishments thus far, and work remaining to be done. The principal tasks that were addressed are:

- Develop needed simulation tools. At the beginning of this effort the existing Monte Carlo simulation tools either did not generate fission products or they generated a single "average" fission product that did not simulate the distribution of events that occurs during the interrogation process. Equally important, it was necessary to assemble a database describing the yield distribution of fission products in $\mathrm{Z}$ and $\mathrm{A}$, and to access the databases of decay schemes for each of those fission products to predict the yield and spectra of subsequent Gray emission. Development of the basic physics model and assembly of databases began under LDRD sponsorship and implementation in the COG code began under DoE/NA-22 sponsorship. Implementing these capabilities in a parallel processor was completed under the current DHS support. Details of these developments have been described elsewhere[10-12].

- Develop the needed experimental facilities. It was necessary to establish a laboratory where realistic cargo assemblies could be assembled at full scale and scanned. The facility needed the capability to field realistic and significant amounts of SNM and to interrogate those systems with a well-characterized neutron beam.

- Evaluate various detector configurations and design an optimum set.

- Evaluate the efficacy of various neutron interrogation sources. Design and install an optimized source.

- Validate the simulation tools using realistic assemblies of SNM, cargo, and detectors.

- Identify and characterize interferences that would reduce SNM detection probability and/or generate false positives.

- Determine the impact on detection and false alarms due to variations in background radiation.

- Establish the conceptual design for an efficacious system and assemble a lab prototype with suitable components.

- Test a prototype in a realistic field environment and evaluate its performance.

\subsection{Enhancement of the Monte Carlo simulation code COG}

As discussed in section 1.3, detection of fissile material with our system rests on observing $\square$-rays emitted following the $\square$-decay of fission fragments. An accurate account of the spectral and temporal evolution of these photons is essential to enabling mature statistical detection efforts aimed at finding evidence of fission in noisy spectra. The ability to simulate transport of post-fission photons is also important for making predictive estimates and comparisons between experiment and calculation. 
COG, the LLNL Monte Carlo code we use for cargo simulations, has been modified to reliably represent the production, transport, and detection of post-fission photons. This code now boasts the unique ability to track a neutron from its birth, account for fission induced by this neutron, generation of specific fission product nuclides, and transport photons emitted as nascent fragments decay to stability. This is a non-trivial capability in part because post-fission spectra are comprised of many thousand lines, each of which can decay with a different characteristic timescale. This is illustrated in the figure below. The top half of this figure shows the spectrum of photons generated by COG to account for photons emitted between 5 and 6 seconds following thermal neutron-induced fission of ${ }^{235} \mathrm{U}$. COG's representation of photons emitted between 30 and 31 seconds following fission is shown in the bottom half of the figure.
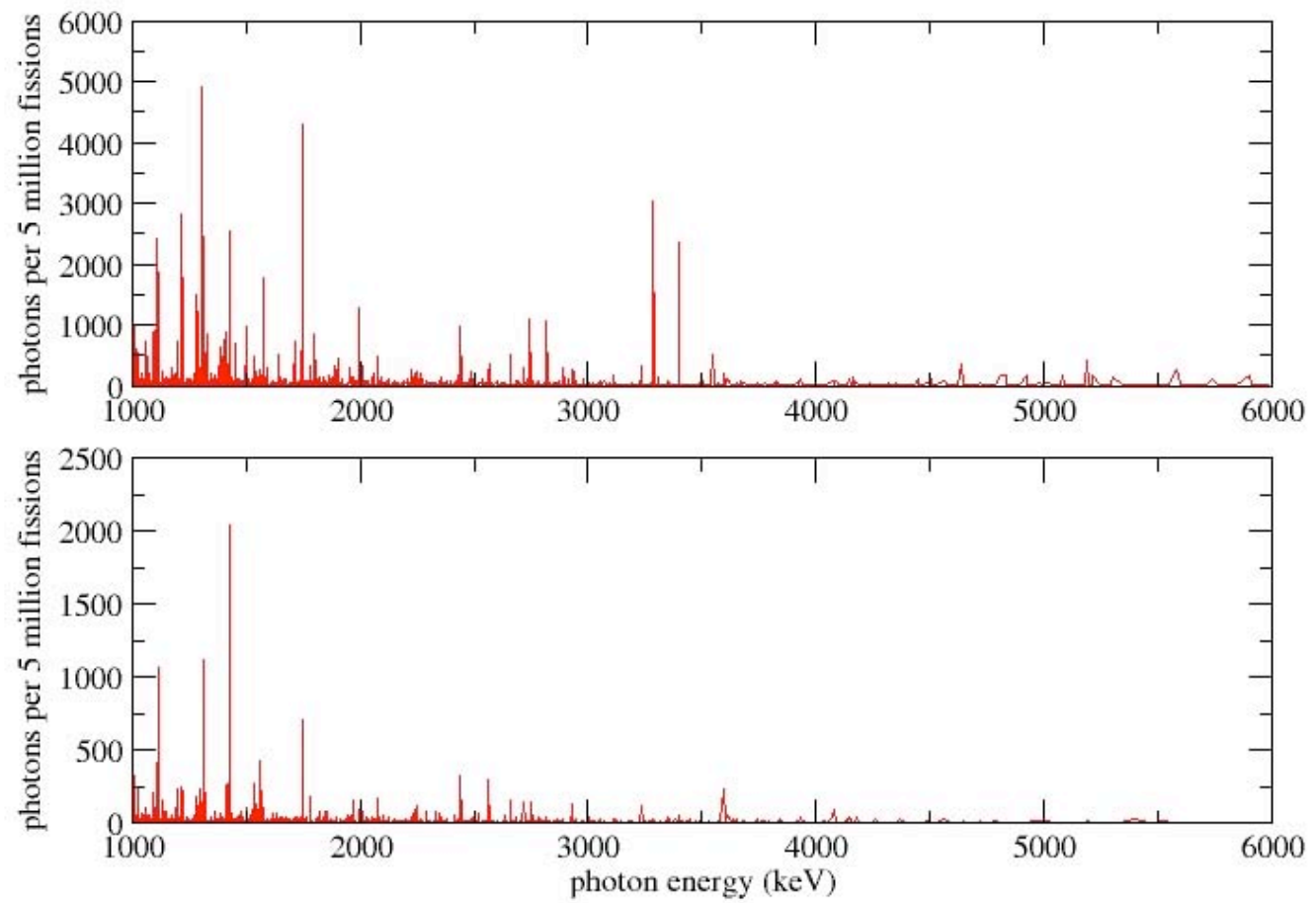

Figure 2.1-1 Spectrum output by COG describing emission of post-fission Grays. The top panel corresponds to photons emitted between 5 and 6 seconds following fission, the bottom to photons emitted between 30 and 31 seconds following fission. Note that weak lines barely distinguishable by eye in these plots account for about half of the overall photon emission.

A considerable science effort went into developing data sets describing emission of post-fission photons by the originators of the idea of using delayed gammas as a signature of clandestine weapons material, coordinated a series of careful experiments at LBNL[6]. These were designed specifically to observe spectra under conditions thought to be of interest to cargo interrogation. Through a lab-funded LDRD we subsequently developed a Monte Carlo method for calculating the photon source function describing emission of $\square$-delayed photons that is used in COG. This method invokes direct sampling of the independent fission yield distributions of the fissioning system, the branching ratios for decay of each fission product and the spectral distributions 
characterizing photon emission for each decay mode. In all, experimentally-derived data describing some 1000 nuclides, 100 isomers and 21,000 individual Gray transitions went into the calculation of the source functions[12].

Examination of the figure above shows something quite new in Monte Carlo simulations. The enhanced COG is now able to simulate the generation of individual Gray lines from individual fission product nuclides. That capability did not exist in simulation codes prior to this augmentation.

Agreement between these calculations and the LBNL experiments was found to be excellent. So that the broader community might benefit from our data efforts we submitted a new format proposal to the ENDF community[13]. This has been accepted and will allow modelers using MCNP to carry post-fission photons in their calculations.

\subsection{Construction of a laboratory for component testing and code validation}

A laboratory has been designed and implemented where components of a detection system can be evaluated operationally in an environment that realistically simulates cargo at a seaport. This laboratory has been utilized first to experimentally validate the simulation tools that will be utilized in the engineering design of a field system at a port, and then it has been utilized to evaluate and demonstrate the performance of the various system components.

\subsubsection{Lab infrastructure}

The cargo-scanning lab has been constructed in the high bay of building 231 at LLNL. That area provides a large floor space where a fully loaded $20 \mathrm{ft}$ cargo container can be positioned and scanned back and forth in front of neutron sources and detectors.

There is a large pit below the floor to facilitate positioning either neutron sources or detectors below the floor. Utilities including electrical power and water are readily available in the work area and a 30-ton overhead crane and heavy-duty forklifts are available as well. There is also storage space for various cargo loads and for an operator control/data acquisition space. A large floor area provides for deployment of a detector array that is longer than the container if needed. A data acquisition system has been commissioned that can digitize signals coming in from 64 channels to support a very large number of detectors and diagnostic equipment such as neutron flux monitors and dosimeters.

The acquisition system provides the capability to record both the pulse height and the arrival time of signals on all of the data channels and to save this in a large master data file where every detected event is archived. This capability allows us to re-run an experiment off-line to evaluate alternative detector configurations or alternative parameter settings without requiring another run of the actual irradiation. The acquired data may then be sorted off-line to provide pulse height spectra from each detector, or count rates vs. time from each detector with variable pulse height discriminator levels, and it provides the capability to sort the data into a 2-D configuration where the data is displayed as a surface with a arrival time axis and a pulse height axis. The latter 2-D configuration allows fitting a surface function to emphasize the fission 
product data and suppress background and interference radiation to improve detection of the SNM.

Soon there will be a trolley system that provides automatic translation of a fully loaded container past neutron sources and/or detector arrays. This system will provide the capability to provide a constant scan speed and to vary that speed over the range $0.06-0.6 \mathrm{~m} / \mathrm{s}$. The highest speed will simulate scanning a $40 \mathrm{ft}$ container in $\sim 20 \mathrm{sec}$.

Below are photos showing the laboratory layout and some components of the infrastructure, detectors, and sources.

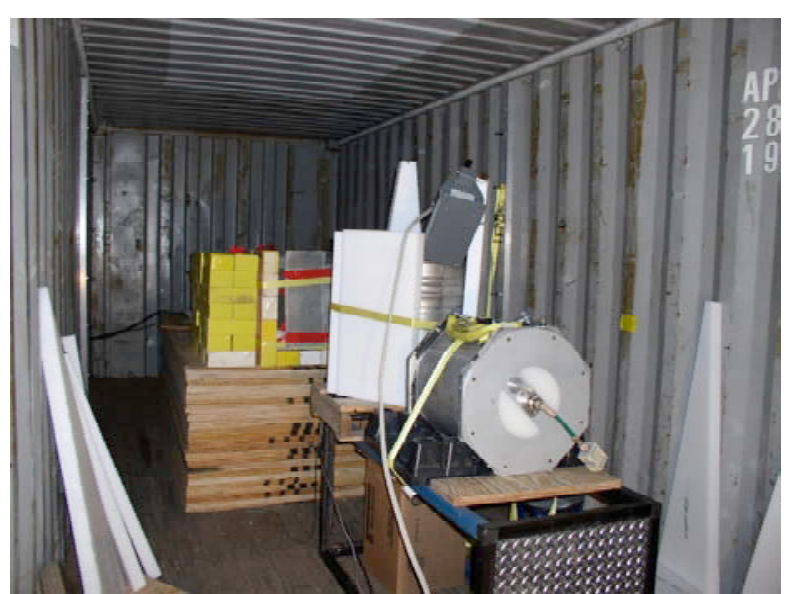

(A)

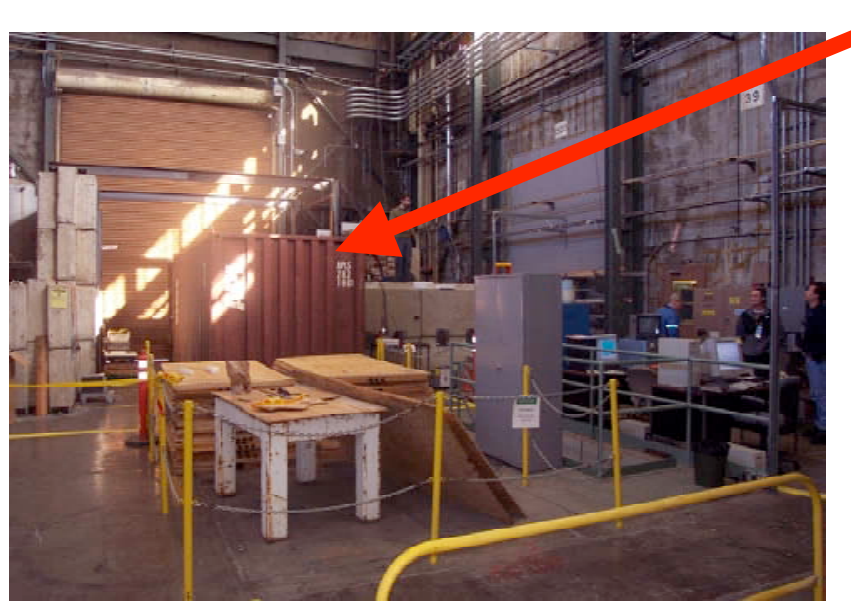

(b)

Figure 2.2.1-1 Laboratory setup in the cargo scanning facility. a) Shows long counter inside a cargo container (foreground) and a collimated HPGe detector in lead shield house on far side. b) Shows the cargo container positioned for a measurement.

\subsubsection{Targets}

The experimental facility is permitted for handling HEU samples with masses up to $800 \mathrm{~g}^{235} \mathrm{U}$. There is a variety of HEU samples available including:

- HEU metal (97\% enriched) with $50 \mathrm{~g}^{235} \mathrm{U}$

- $\quad$ HEU oxide (94\% enriched) $250 \mathrm{~g}^{235} \mathrm{U}$

- $\quad$ HEU oxide (94\% enriched) $380 \mathrm{~g}^{235} \mathrm{U}$ in powder form

- These targets can be used separately or together to provide targets up to the maximum allowed mass.

- Also available are samples of natural uranium beads up to $22 \mathrm{~kg}$ that can be installed in containers of various shapes to evaluate alternative target geometries,

- And there are samples of depleted uranium (DU) with masses of hundreds of $\mathrm{kg}$. 


\subsubsection{Cargos}

There is an enormous range of cargo materials and geometries passing through seaports, as described in Section 1.1. For a development effort such as the one described here it is necessary to distill the range of cargos down to a few representative surrogates. Making a judgment based on the data in the introduction we choose mean density is $\square \leq^{\prime} 0.6 \mathrm{~g} / \mathrm{cm}^{3}$. Our surrogate cargos strive toward the upper bound density. A large fraction of the cargos in transit contain organic materials in the form of agricultural products, fuels, plastics, and other hydrocarbons. A significant fraction of the cargos contain electronics components. And some contain machinery. The LLNL cargo-scanning lab has three basic cargos to act as surrogates for the overall traffic. They are:

- Plywood sheets, $120 \times 240 \mathrm{~cm}$ and $0.95 \mathrm{~cm}$ thick. When dry this material has density $0.65 \mathrm{~g} / \mathrm{cm}^{3}$. Its chemical composition is primarily $\mathrm{H}, \mathrm{C}$, and $\mathrm{O}$. This composition is strikingly similar to that of most agricultural products, fiber products, and plastics. Its radiation transport properties bear a reasonable resemblance to fuels as well. There is enough plywood available in the cargo-scanning lab to fill a $20 \mathrm{ft}$ container.

- Aluminum sheets, $120 \times 240 \mathrm{~cm}$ and $0.32 \mathrm{~cm}$ thick. Electronics components are made up (on a weight basis) primarily of aluminum, plastic, and glass. Aluminum and silicon are adjacent in $\mathrm{Z}$ and so we take aluminum sheets to be a reasonable radiation transport surrogate for electronics components. To represent a cargo shipment the sheets are separated by spacers so that the mean density is $\sim 0.6 \mathrm{~g} / \mathrm{cm}^{3}$.

- Steel pipes, $10 \mathrm{~cm}$ diameter x $6.4 \mathrm{~mm}$ wall thickness. The pipes are stacked into an array with axes alternating in direction as one goes from one level to the next. The choice of diameter, wall thickness, and stacking pattern leads to a mean iron density of $\sim 0.6$ $\mathrm{g} / \mathrm{cm}^{3}$.

The three basic cargo materials described above are stacked onto standard pallets to a height typically $150-200 \mathrm{~cm}$ and loaded into a standard $20 \mathrm{ft}$ cargo container. They are illuminated from below by the neutron source and the subsequent fission product $\square$-decay radiation is detected by a detector array along the side of the container.

The figures below show some of the cargos used in experiments described. 


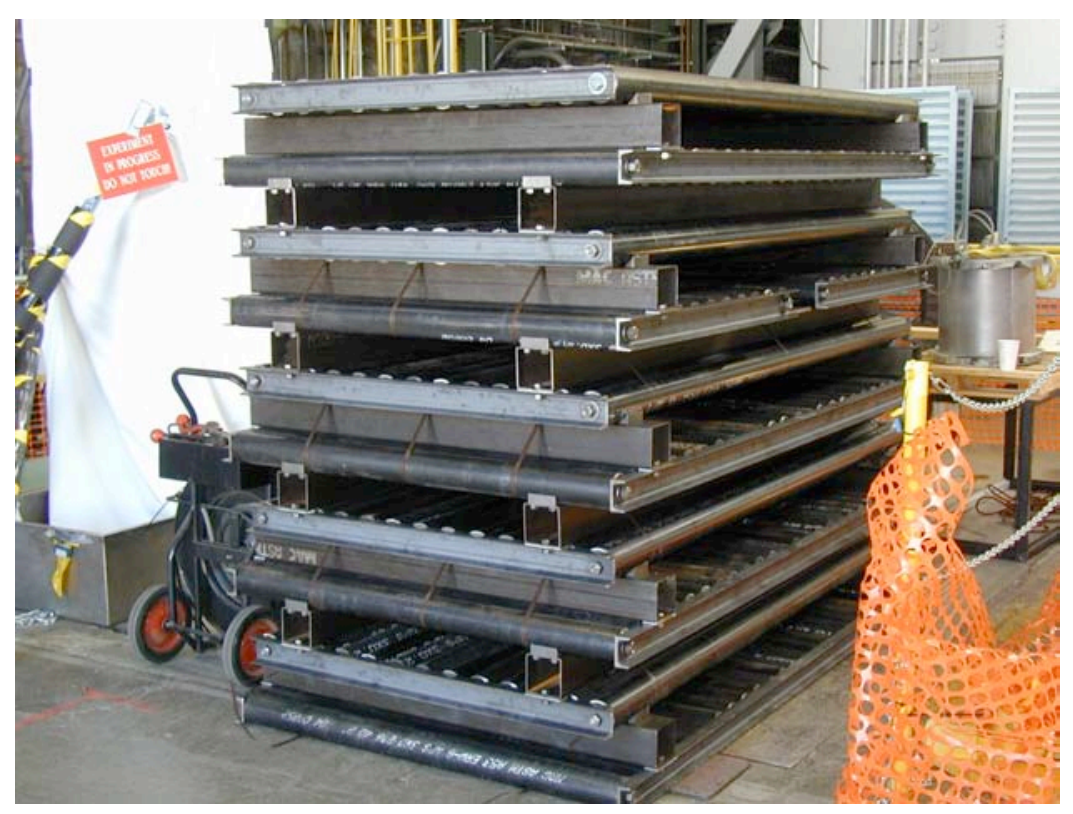

Figure 2.2.3-1

Steel cargo surrogate

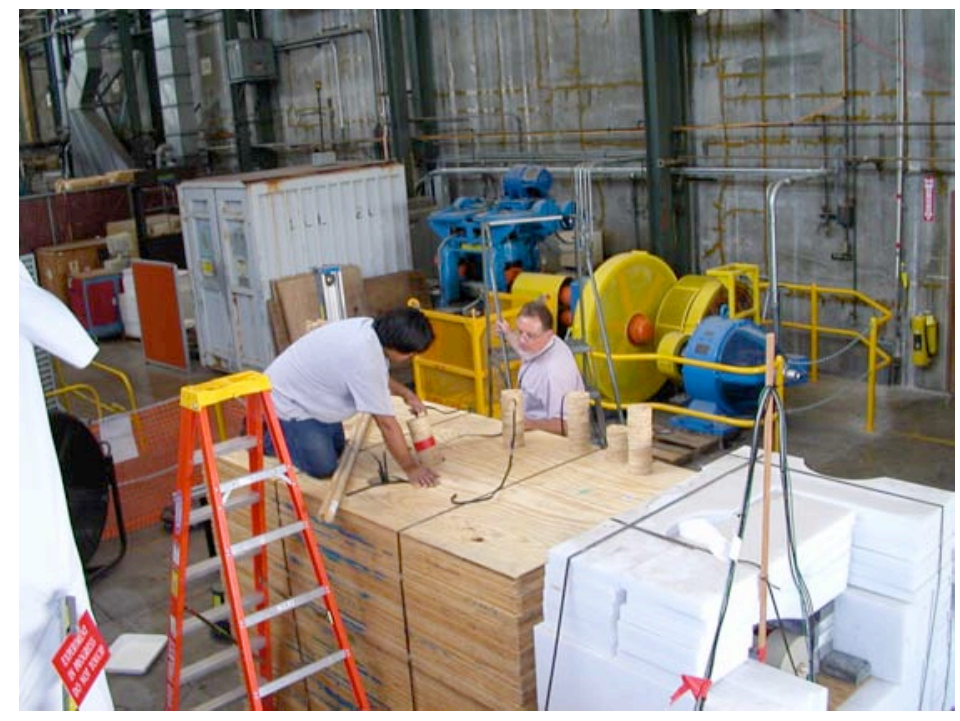

Figure 2.2.3-2

Wood cargo surrogate

\subsubsection{Detectors}

A variety of radiation detectors have been installed in the cargo-scanning lab to provide diagnostics on the neutron source, dosimetry for personnel protection, and measurement of $\checkmark$-radiation that is compared to simulations and to demonstrate component performance.

- The detectors include an array of four large liquid scintillators with active volume $20 \mathrm{~cm}$ diameter and $183 \mathrm{~cm}$ length. There is a $20 \mathrm{~cm}$ photomultiplier (PMT) at each end of 
each cell. Development of these detectors began under LDRD funding, continued under DoE/NA-22 funding, and were completed within the present project.

- There are several plastic scintillators with active volume $61 \mathrm{~cm} \mathrm{X} 122 \mathrm{~cm}$ and thicknesses $15 \mathrm{~cm}$ and $25 \mathrm{~cm}$.

- Some of the plastic scintillators have area $61 \mathrm{~cm} \mathrm{X} 61 \mathrm{~cm}$

- Some of the plastic scintillators have two PMT's on one end, others have two PMT's on the large surface, and others have only a single PMT either on the large surface or one of the small surfaces.

- There is a $68 \%$ HPGe high-resolution Gray spectrometer. It is well collimated and shielded from the primary neutron interrogation beam. It is utilized to identify activation products and fission products. It is used to determine the absolute Gray intensity and to determine the number of fission products generated, and from that the fission rate for comparison with simulations.

- There is a dePangher long counter for accurate and absolute measurements of neutron flux. Its response is nominally energy independent and it is used to calibrate other detectors as well as to provide a benchmark for simulation validation.

- There are several small plastic scintillators coupled to PMT's and located immediately adjacent to the neutron source to continuously monitor its output. These monitors are calibrated against the long counter.

- There are several ${ }^{3} \mathrm{He}$ proportional counters. Some are bare and others in polyethylene moderators. These detectors are used as flux monitors in order to map the spatial profile of neutrons illuminating the cargo, and can be utilized to detect fission product delayed neutrons.

- There are polyethylene $\mathrm{REM}$ meters, each with a small $\mathrm{BF}_{3}$ proportional counter embedded in a specially designed polyethylene moderator. These are utilized to record environmental dose rates and sometimes as neutron beam monitors.

Below are photos of some of the detectors as they are used in the experiments described here. 


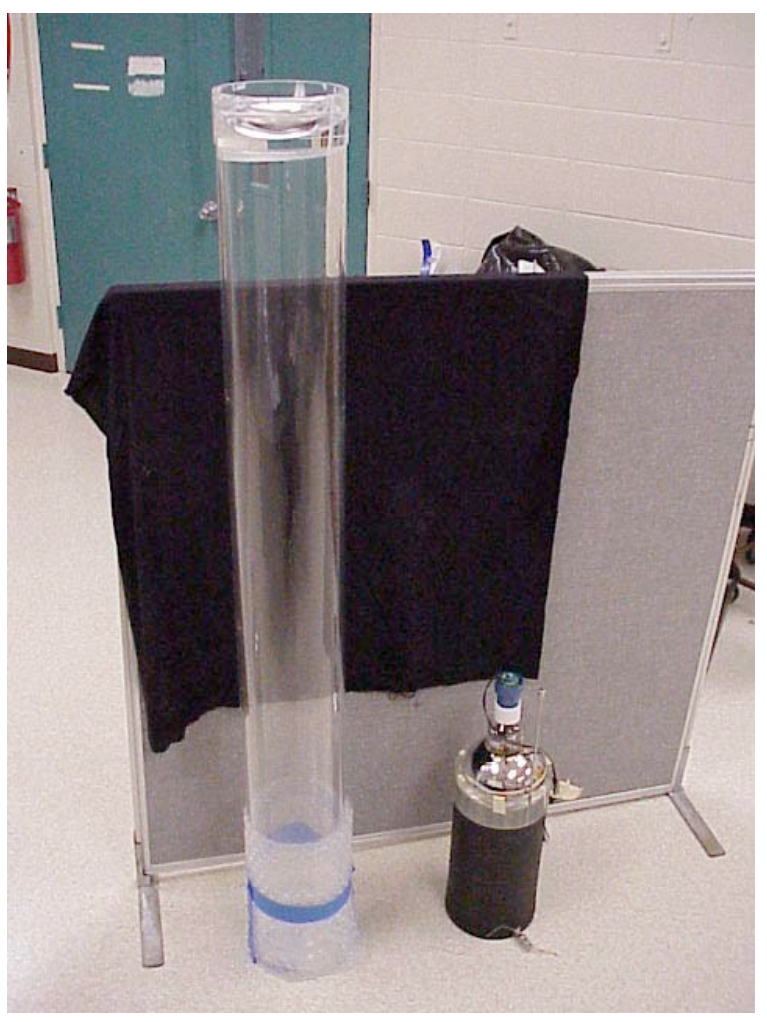

(a)

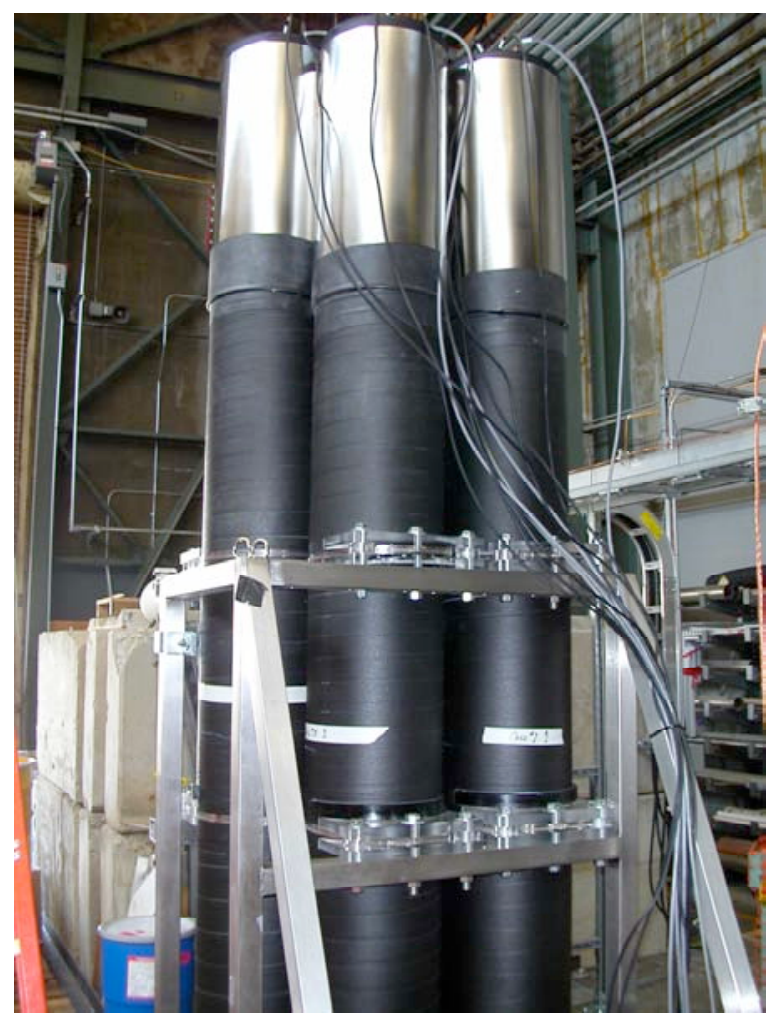

(b)

Figure 2.2.4-1 Liquid scintillation detectors. a) Detector ready for liquid fill up. b) Detector in place near experiment.

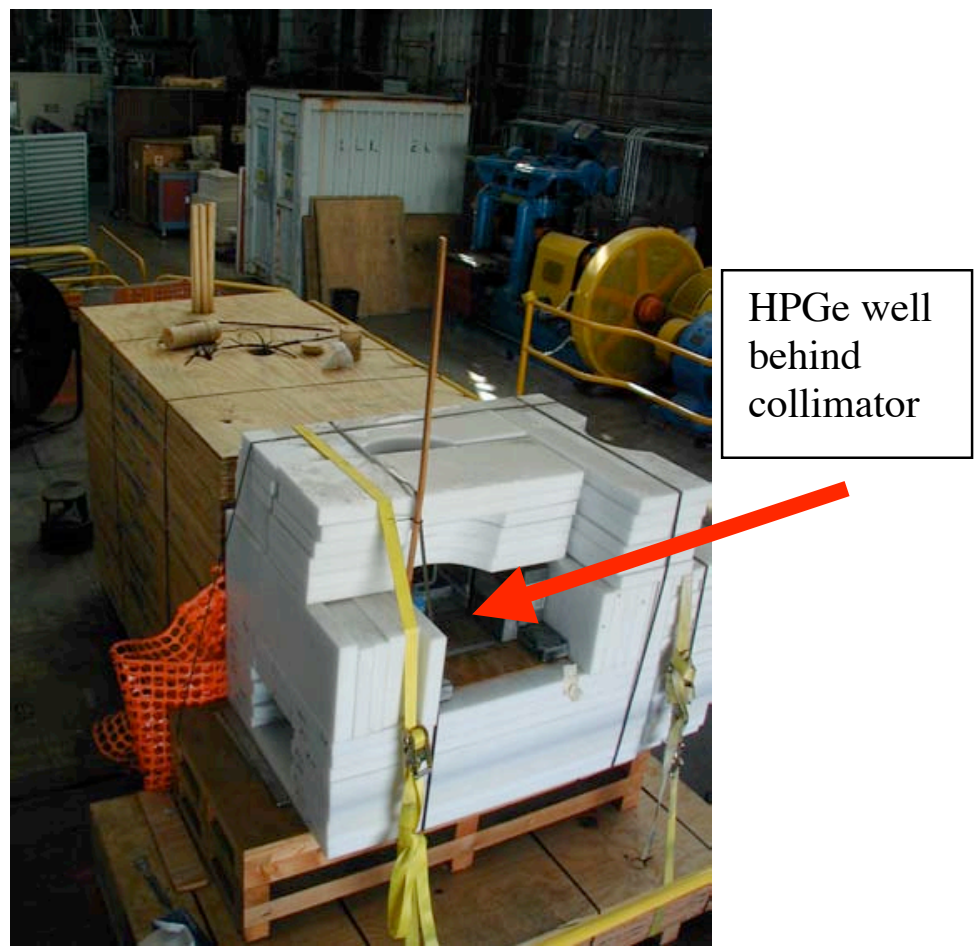


Figure 2.2.4-2 HPGe spectrometer collimator deployed on one side of the plywood cargo.

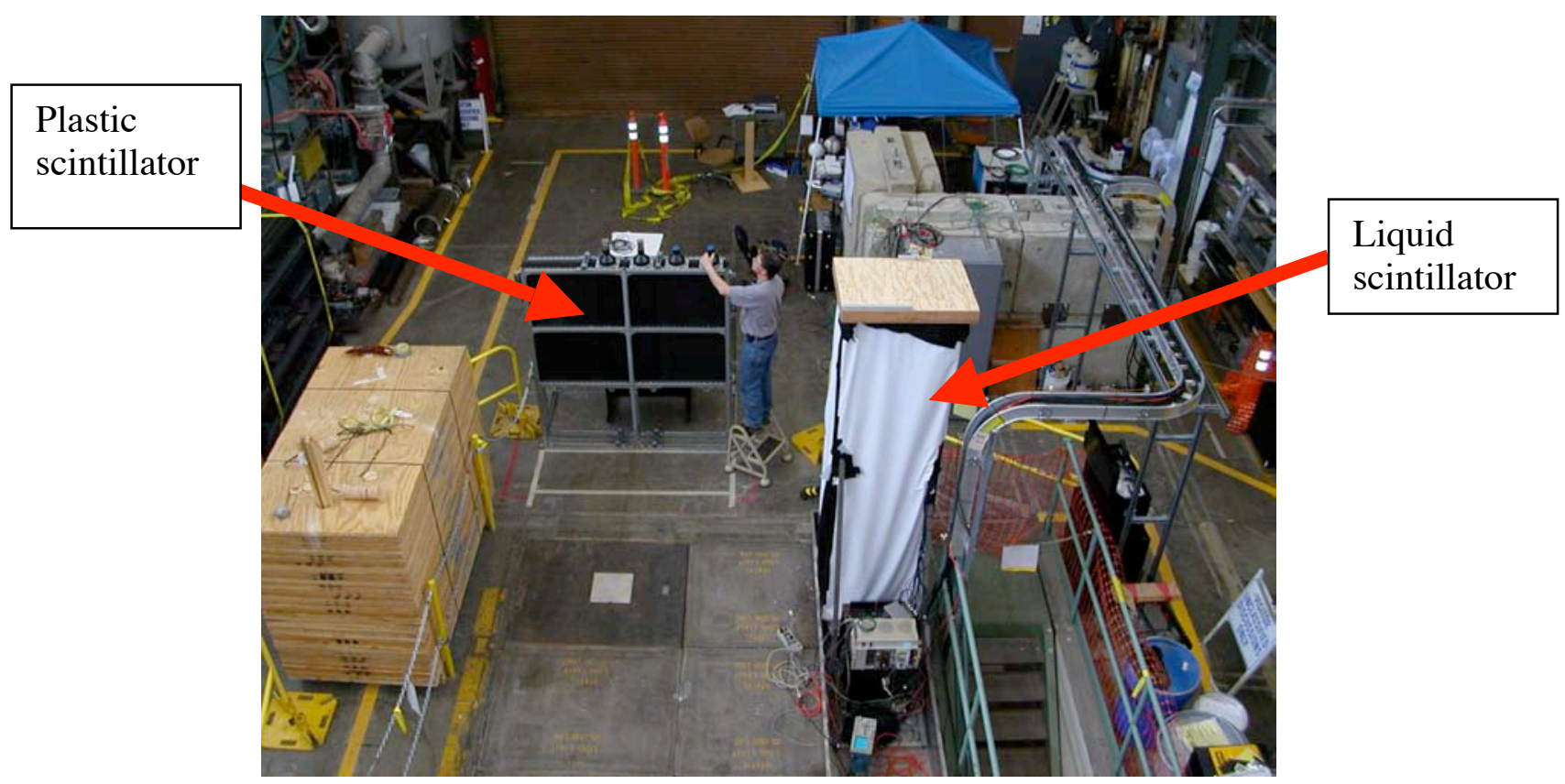

Figure 2.2.4-3

Plastic detectors deployed for a cargo measurement.

\subsubsection{Neutron sources}

There are a variety of neutron sources available in the cargo-scanning lab. They include:

- Commercially available D-T neutron generators sold by VNII that produce $14 \mathrm{MeV}$ neutrons at rates up to $\leq 1 \times 10^{9} \mathrm{n} / \mathrm{s}$. These were acquired under DoE/NA-22 funding.

- Commercially available D-D neutron generators sold by VNII that produce $2.5 \mathrm{MeV}$ neutrons at rates to $\leq 1 \times 110^{7} \mathrm{n} / \mathrm{s}$. These were acquired under DoE/NA-22 funding.

- ${ }^{252} \mathrm{Cf}$ neutron sources that produce a fission spectrum with total output $1 \times 10^{5} \mathrm{n} / \mathrm{s}$.

- Commercially available D-D neutron generator made by Kaman Sciences (A711) that produces $2.5 \mathrm{MeV}$ neutrons at rates up to $\leq 1 \times 10^{9} \mathrm{n} / \mathrm{s}$. This generator was refurbished under DoE/NA-22 funding.

- Commercially available D-T neutron generator made by Kaman Sciences (A711) that produces $14 \mathrm{MeV}$ neutrons at rates up to $\leq 1 \times 10^{11} \mathrm{n} / \mathrm{s}$. Operating at a typical output $6 \times 10^{10} \mathrm{n} / \mathrm{s}$ the neutron flux at $\mathrm{R}=2.5 \mathrm{~m}$ is $\square \sim 8 \times 10^{4} \mathrm{n} / \mathrm{cm}^{2} / \mathrm{sec}$. It was refurbished under DoE/NA-22 funding.

- Commercially available RFQ accelerator that produces a well-focused beam of $4 \mathrm{MeV}$ deuterons at beam current $100 \square \mathrm{A}$. 
- $\quad{ }^{13} \mathrm{C}$ target where the ${ }^{13} \mathrm{C}(\mathrm{d}, \mathrm{n}){ }^{14} \mathrm{~N}$ reaction produces a broad spectrum of neutrons in the range $4-11 \mathrm{MeV}$ with output $\sim 5 \times 10^{11} \mathrm{n} / \mathrm{s}$.

- A deuterium gas target that produces a neutron beam whose spectrum is roughly constant over the range 3-7 MeV and whose angular distribution is highly peaked in the forward direction. This generator can produce a flux on axis at $\mathrm{R}=2.5 \mathrm{~m}$ up to $\square \sim 1 \times 10^{6} \mathrm{n} / \mathrm{cm}^{2} / \mathrm{sec}$.

All of the above sources can be pulsed and provide for interrogation followed by detection of the fission product radioactivity.

Each source can be installed in a pit below the floor of the scanning lab. The pit depth and transverse dimensions provide a variety of positioning strategies but usually the source is mounted close to floor level. The earth below the floor provides shielding on two sides and usually wood or polyethylene shielding is installed on the other two sides to reduce personnel dose rates. In most cases the neutron sources are collimated with structures containing $\leq 2$ tons of steel surrounded by 0.5-1.0 tons of polyethylene. Collimation reduces personnel dose in the experimental area and can provide beams with principal intensity limited to a $60 \times 60 \mathrm{~cm}$ spot at the target location. Details of the collimation are given later in Section 3.2.

The figure below shows the collimator in the floor with the D-T source at the entrance to the collimator.

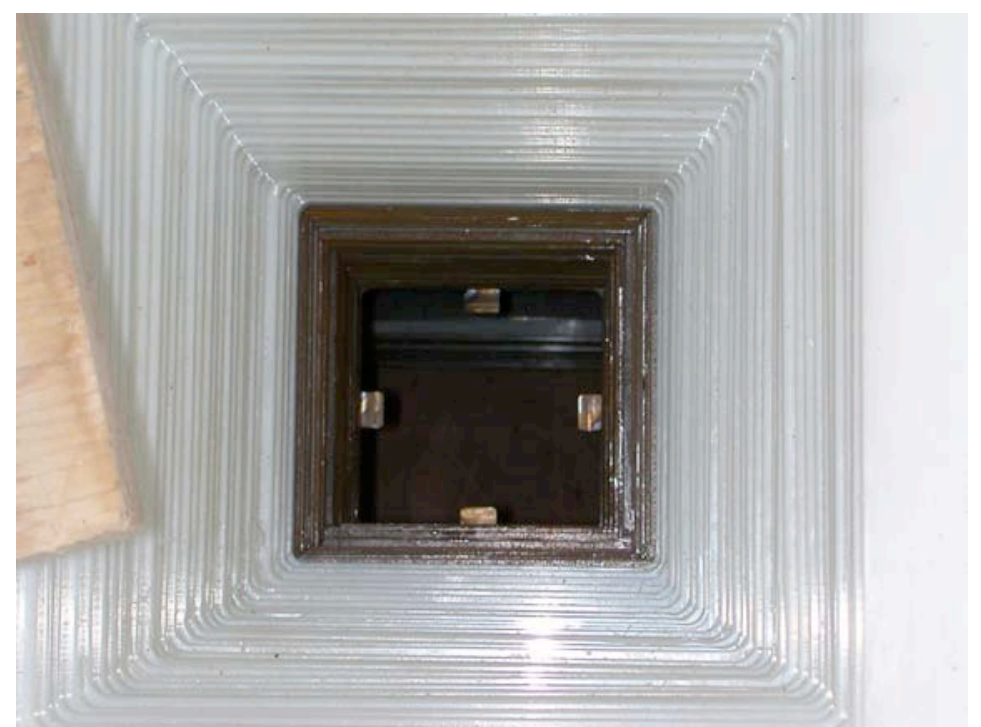

Figure 2.2.5-1 Top view of neutron source with steel/polyethylene collimator

\subsubsection{The RFQ neutron source}

The highest neutron flux obtainable from the isotropic sources at $\mathrm{R}=2.5 \mathrm{~m}$ was $\square " \sim " 5 \times 10^{4 "} \mathrm{n} / \mathrm{cm}^{2} / \mathrm{sec}$. Rough estimates based on hand calculations and extrapolations from experimental data indicate that meeting the detection requirements in a short scan of $\leq 1$ minute demands a neutron flux at $\mathrm{R}=2.5 \mathrm{~m}$ on the order of $1-10 \times 10^{6} \mathrm{n} / \mathrm{cm}^{2} / \mathrm{sec}$. A new source 
technology is required. The D-D reaction with an energetic beam of deuterons provides several major benefits:

- The neutron energy is much higher than that produced by sealed source D-D machines and thus penetrates the cargo well compared to $2.5 \mathrm{MeV}$ neutrons.

- The angular distribution is highly peaked in the forward direction, increasing the flux on axis.

- The $\mathrm{D}(\mathrm{d}, \mathrm{n})$ reaction cross sections rises rapidly with deuteron energy up to a maximum at $\mathrm{E}_{\mathrm{d}} \sim 5 \mathrm{MeV}$.

The figure below illustrates this.

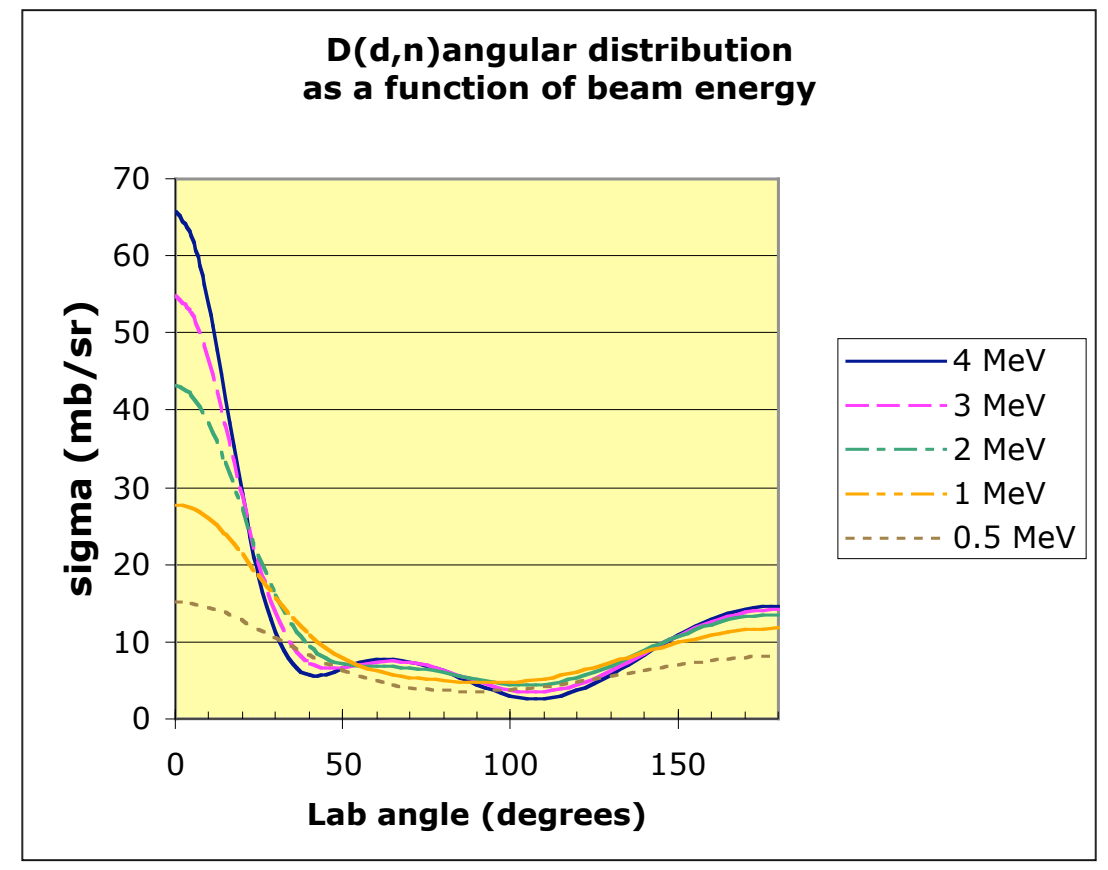

Figure 2.2.5.1-1

Angular distribution for the $D(d, n)$ reaction at several deuteron beam energies.

Note that for a $4 \mathrm{MeV}$ incident beam the half-width of the outgoing neutrons is only 25 degrees. This not only increases the neutron flux on-axis, but it also reduces the shielding requirements around the target as the output is distinctly suppressed normal and at large angles to the beam. Similarly, the reaction cross-section rises with incident beam energy as seen in the figure below. 


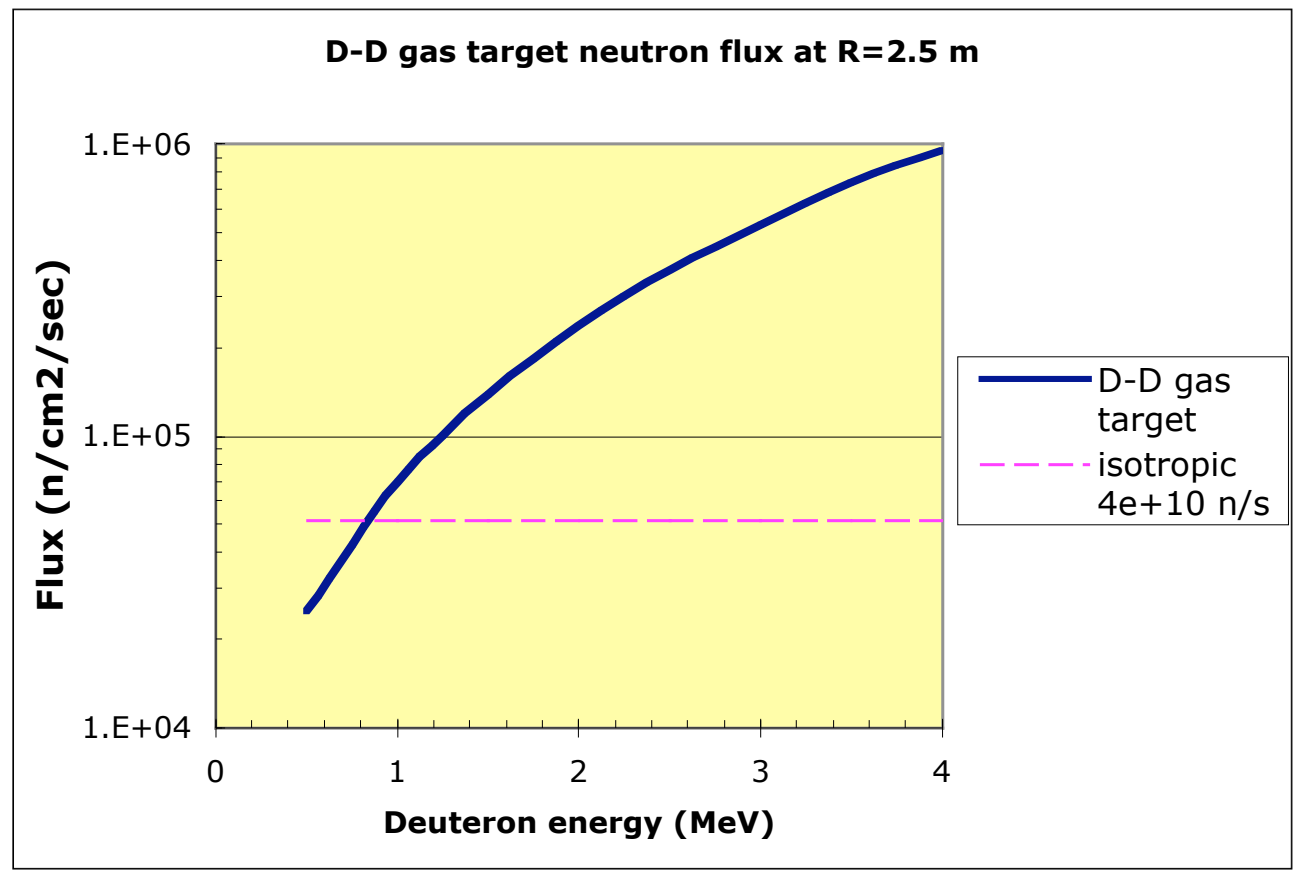

Figure 2.2.5.1-2 Estimated neutron flux on-axis at $R=2.5 \mathrm{~m}$ for (a) an isotropic source with output $1 \times 10^{11} \mathrm{n} / \mathrm{s}$, and (b) for the $D-D$ reaction. For the latter the target is assumed to be a gas target of thickness sufficient to nearly stop the beam and the beam current is assumed to be $100 \square$ A.

Examination of the figure shows that a $100 \square \mathrm{A}$ deuteron beam at $4 \mathrm{MeV}$ incident on a thick gas target provides a roughly $15 \mathrm{X}$ increase in flux on target compared to the brightest commercially available sealed source. For this reason an RFQ accelerator has been installed in the below ground pit to provide this beam onto a $1 \mathrm{~atm}$ gaseous deuterium target.

\subsubsection{RFQ generator installation}

An RFQ has been installed in the lab pit and a beam transport system has been installed to bring the beam into the vertical orientation and transport it to the gas target immediately below the floor. The target, in turn, is enclosed by a collimator modified slightly from its usage with the earlier D-T generator. A photograph of the installation is shown below. 


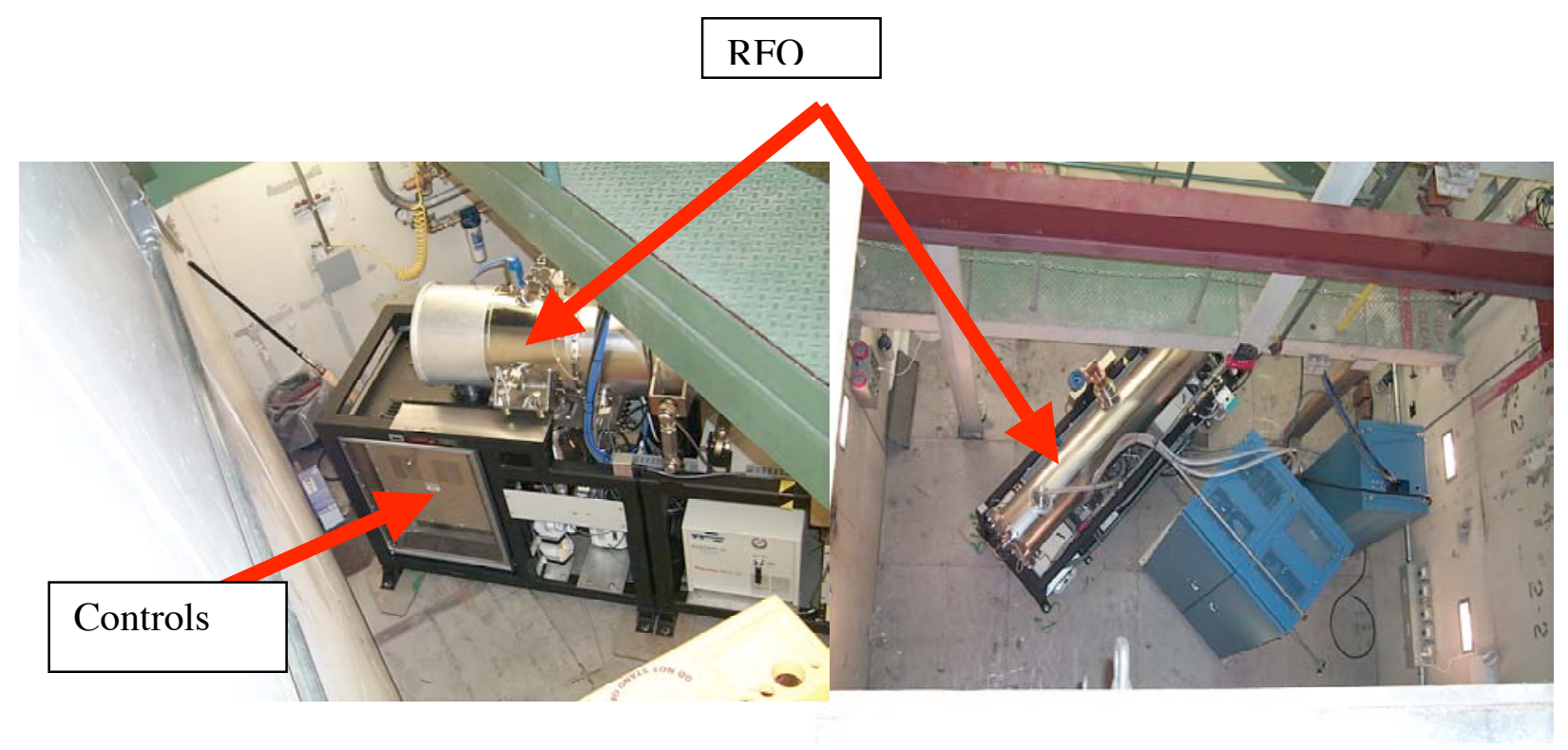

Figure 2.2.5.2-1 Installation of RFQ neutron source in cargo scanning lab. 


\section{Qualification of a Computational Model utilizing a $14 \mathrm{MeV}$ neutron source}

\subsection{Background}

The principal thrust of the cargo project at LLNL is to determine the extent to which neutron interrogation of sea-going cargo containers might be effective for detecting the presence of clandestine fissionable nuclear material. Given the wide-range of cargos that must be considered and practical constraints on the number and type of experiments that can be performed, a central goal of the project is the development and qualification of a Monte Carlo model that can predict experimental outcomes with high accuracy.

For this purpose, a combination of numerical modeling and experiments have been undertaken to compare a baseline Monte Carlo model with experimental fact, the model is implemented in the COG code of LLNL[14]. This entailed comparisons of predicted and experimental measurements on the spectral distributions and strengths of the neutrons obtained from the source, the fission rates induced in HEU specimens in the absence of surrounding cargos, fission rates in HEU specimens surrounded by representative cargo materials and the intensity and spectral distributions of delayed Grays incident on and interacting with scintillation detectors.

The following is meant to provide an accurate summary of the experiments and modeling, including those of the ancillary measurements required for calibration of the detectors and monitors. It represents a fusion of information provided in the basic reports on experiments and calculations. However, the quantitative information provided here often differs from that in the basic reports because of corrections for factors not known with certainty at the time of the initial analyses, more realistic treatment of experimental data and/or more realistic treatment of experimental errors.

Simulations with the baseline model provide spectral distributions and absolute intensities of neutrons at various locations, the number of fissions produced in the presence of a fissionable target, the intensity and spectral distributions of delayed $\square$ rays from decay of the fission products, the intensity and spectral distribution of photons penetrating various cargos due to the delayed $\square$ rays and the spectral distribution and intensity of the interactions of the penetrating $\checkmark$-rays in prototype detector systems, all normalized to a unit neutron source. As a result, qualification of the baseline model can be considered in two relatively independent parts, the first entailing comparisons of the simulated spectral distributions with experimental measurements and the second entailing comparisons of the absolute intensities per source neutron. For each stage in the qualification process, comparison of relative magnitudes is considered first, followed by the comparison of absolute quantities.

\subsection{The Laboratory and Neutron Source}

The experimental facility is housed on the main floor of the Building 231 High Bay and in a pit below floor level located near the north end of the building. Some of the details were given in Section 2.2 above. The baseline model used in the simulations contains a detailed representation of the main aspects of the facility[15] and a schematic of the pit in which the generator is housed is shown in Figure 3.2-1. 

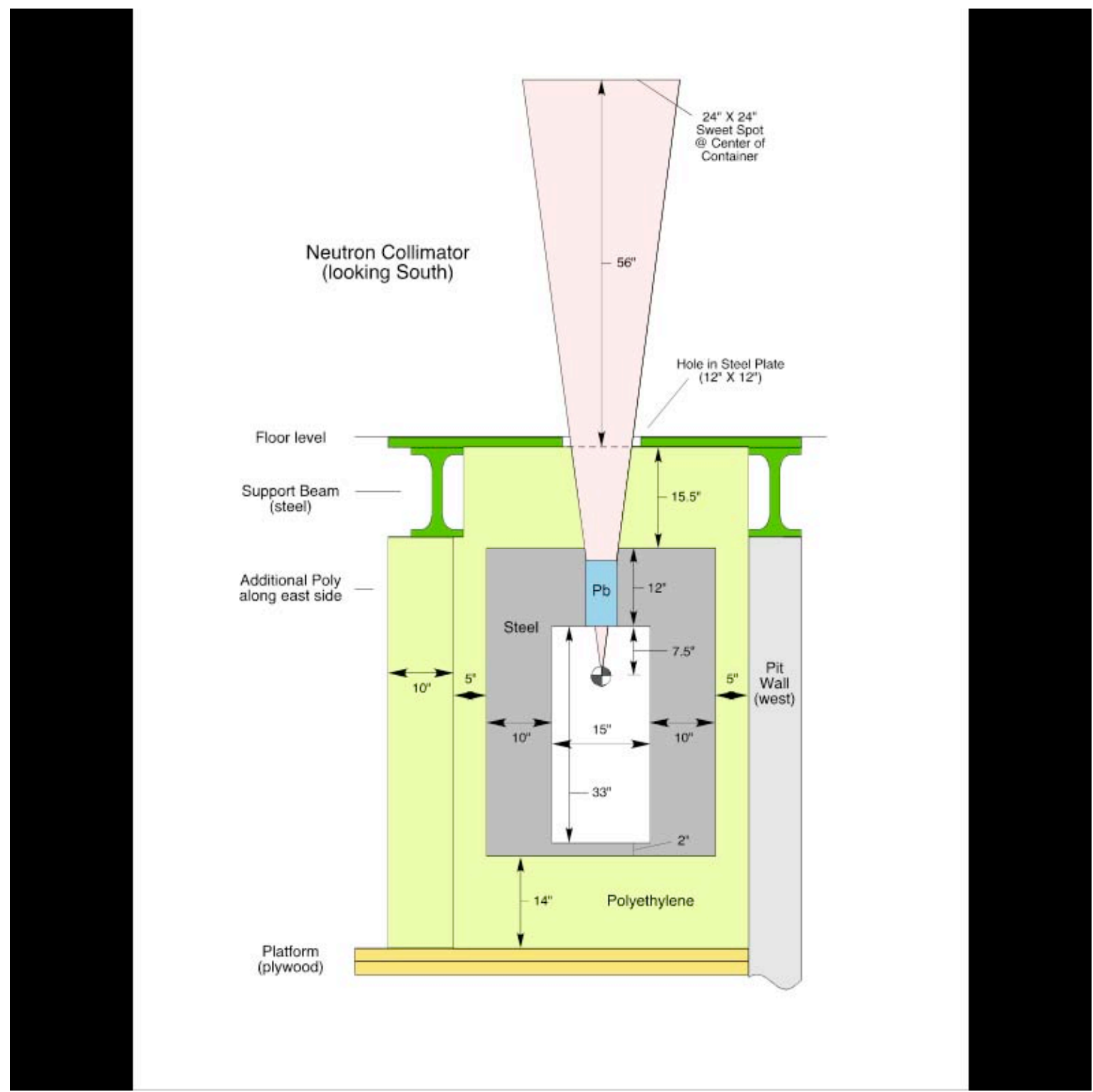

Figure 3.2-1. Schematic representation of the collimator geometry of the neutron generator collimator in the pit below the floor level of the experimental laboratory. As shown, the collimator contains a Pb plug in the vicinity of its throat that was not used in the present experiments.

The neutron source used in our initial experiments is a refurbished ${ }^{3} \mathrm{H}(\mathrm{d}, \mathrm{n})^{3} \mathrm{He}$ neutron generator originally manufactured by Kaman Sciences, Inc. neutron generator Model A711. It has a nominal maximum output of $1 \times 10^{11} \mathrm{~ns}^{-1}$ at an acceleration voltage of $180 \mathrm{kV}$ and was normally operated in the range $4-6 \times 10^{10} \mathrm{n} / \mathrm{s}$. It is contained in a collimator constructed of polyethylene and low-carbon steel to provide a beam at the opening of the collimator throat of $30.5 \mathrm{~cm} \mathrm{x} 30.5 \mathrm{~cm}$, to provide extensive shielding and to minimize neutron activation of the surroundings. A target centered at a height of $142 \mathrm{~cm}$ above the collimator opening represents a target located at the midpoint of a standard sea-going cargo container of nominal height $259 \mathrm{~cm}$. 
Neutrons emanating from the collimator throat with energies in the range $\mathrm{E}_{\mathrm{n}}=2 \mathrm{keV}$ to $15 \mathrm{MeV}$ were monitored with a dePangher Long Counter and low-energy neutrons were monitored with a ${ }^{3} \mathrm{He}$ neutron detector. The generator output was monitored with a small plastic scintillation detector located at a fixed location in the generator pit.

\subsubsection{Calibration of the dePangher Long Counter}

The dePangher long counter is described in Slaughter[16] and references contained therein. In the absence of recent tests, calibration of this device was checked with a ${ }^{252} \mathrm{Cf}$ source of strength $(1.52 \pm 0.05) \times 10^{6} \mathrm{~ns}^{-1}$ as of $08 / 05 / 04$. The source strength was determined by comparison with NIST-traceable sources.

The neutron spectrum was considered with respect to two of the functional forms provided by the ESARDA Working Group, the Watts representation

$$
\square(E)=C e^{\square E / a} \sinh (b E)^{1 / 2}, \text { with } \mathrm{a}=1.025 \mathrm{MeV} \text { and } \mathrm{b}=2.926 \mathrm{MeV}^{-1},
$$

and the Maxwell-Boltzmann representation

$$
M B(E)=B \frac{2}{\sqrt{\square} T^{3 / 2}} \sqrt{E} e^{\square E / T}, \text { with } \mathrm{T}=1.42 \mathrm{MeV}
$$

These are shown in Figure 3.2.1-1 with normalization to the peak intensity for comparison purposes. The average energies for the two distributions are $2.306 \mathrm{MeV}$ (Watts) and $2.130 \mathrm{MeV}$ (Maxwell-Boltzmann), respectively, essentially identical for the present purposes. As a result, we have assumed the Watts distribution to represent the ${ }^{252} \mathrm{Cf}$ neutron spectrum.

The energy dependence of the relative sensitivity of the long counter is reported in Slaughter[16] and is reproduced in Figure 3.2.1-2. The efficiency is seen to be roughly constant over the range $.03-1 \mathrm{MeV}$ and then to decrease slowly with increasing energy. In order to provide a reasonable representation of the relative sensitivity, the functional form $I_{r e l}=a_{1} e^{\square a_{2} E}$, where $\mathrm{E}$ is the neutron energy in $\mathrm{MeV}$, was chosen. Included in Figure 3.2.1-2 are least squares fits of this function to the data with and without inverse variance weighting. The former clearly represents the data to a much higher degree and the parameters from this fit are shown below the figure. This fit was adopted for use in all computations requiring knowledge of the energy dependence of the long counter sensitivity.

The detection rate in the long counter for monoenergetic neutrons, $\mathrm{R} \mathrm{ns}^{-1}$, can be expressed as

$$
R=\frac{S}{4 \square d^{2}} \square A
$$




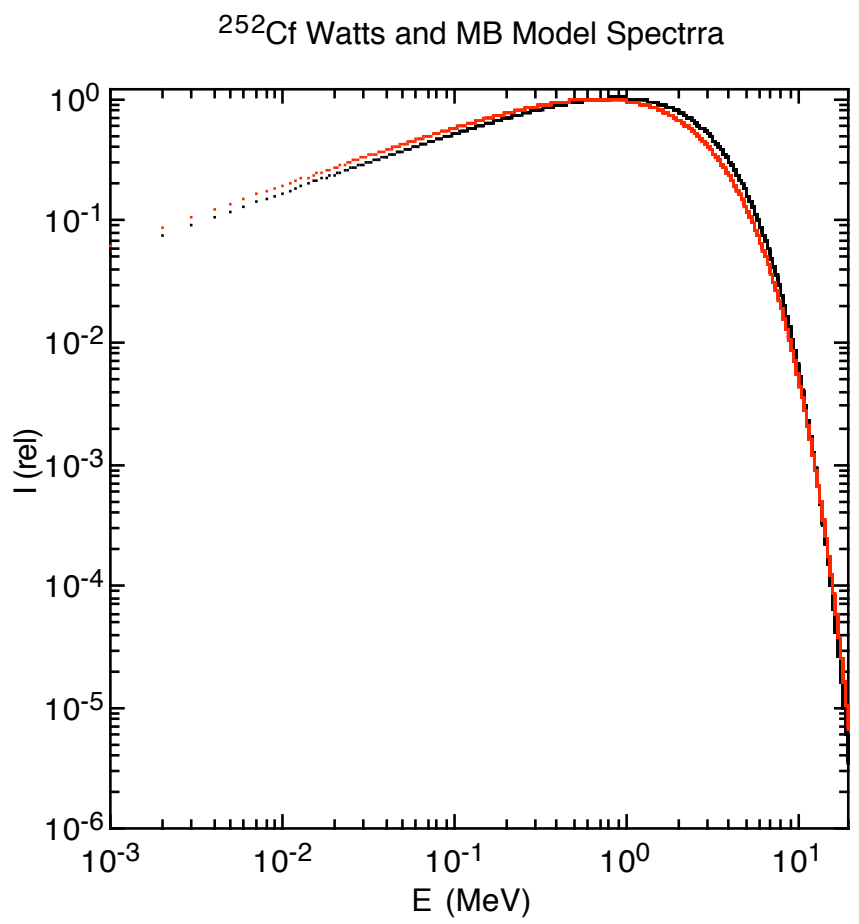

Watt spectrum

Normalized MB Spectrum

Figure 3.2.1-1. The neutron spectrum from fission of ${ }^{252} \mathrm{Cf}$ according to the Watts (black) and Maxwell-Boltzmann (red) distributions.

where $\mathrm{S}$ is the source strength, $\mathrm{d}$ is the distance from the source to the effective center of the long counter, $\square$ is the intrinsic efficiency for detecting a neutron and $\mathrm{A}$ is the cross sectional area of the entrance face of the counter. The product $\square$, with dimensions $\mathrm{ns}^{-1} \square^{-1}\left(\mathrm{~cm}^{2}\right)$ is the absolute sensitivity of the long counter per unit incident neutron flux. Alternatively, $\square$ A can be viewed as the effective area of the long counter.

For any incident spectrum, $n(E)$, the average value $<\square A>$ is given by

$$
\langle\square A\rangle=\frac{\square n(E) \square A(E) d E}{\square n(E) d E}=\frac{4 \square}{S}\left\langle R d^{2}\right\rangle .
$$

The absolute calibration of the long counter was determined through a series of measurements in which the long counter was supported on an aluminum frame in the form of a rectangular parallelepiped located on the steel cover plate of the collimator pit (Figure 3.2-1) The entrance face of the counter was horizontal and faced upward. Under these conditions, the face of the counter was located at approximately $1.2 \mathrm{~m}$ above the floor of the laboratory. The ${ }^{252} \mathrm{Cf}$ source was located on the axis of the long counter at four distances between 38.94 and $130.34 \mathrm{~cm}$ above its effective center[16]. Under these conditions, chosen to minimize detection of scattered neutrons to a reasonable degree, the count rate varied as $1 / \mathrm{r}^{2}$ within experimental error. Shadow shielding with polyethylene showed that scattered neutrons contributed to less than $1 \%$ of the count rate at all distances. 


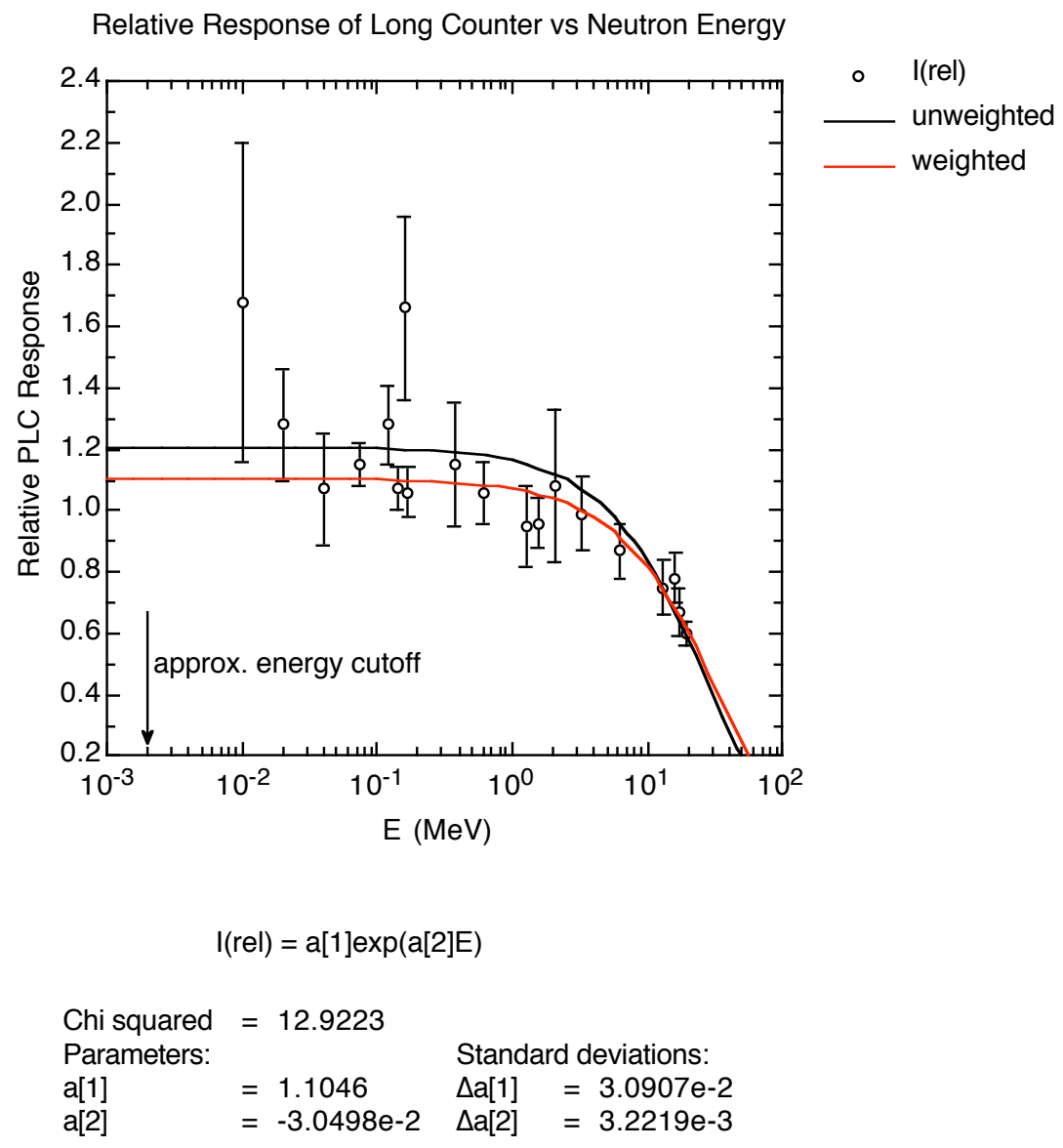

Figure 3.2.1-2. Reproduction of the relative efficiency of the dePangher long counter from Slaughter [16] (data points) along with least squares fits with and without inverse variance weighting of the data. The fitting parameters shown below the figure represent the least squares fit with inverse variance weighting of the data.

From these measurements, after adjusting for a small change in the effective center of the long counter to reflect the average over the Watts spectrum and a more realistic error analysis, the average value of the sensitivity for ${ }^{252} \mathrm{Cf}$ neutrons was determined to be $\square \mathrm{A}=3.43 \pm 0.17 \mathrm{~ns}^{-1} \square^{-1}$. This is taken to represent the sensitivity at the energy of $2.3 \mathrm{MeV}$. The result is in excellent agreement with the previous measurements reported[16]. The functional fit to the relative efficiency data shown in Figure 3.2.1-2 provides the ratio of the sensitivity for $14.25 \mathrm{MeV}$ neutrons to that for $2.3 \mathrm{MeV}$ neutrons of $0.695 \pm 0.042$ and thus an absolute sensitivity for 14.25 $\mathrm{MeV}$ neutrons of $\square \mathrm{A}=2.38 \pm 0.19 \mathrm{~cm}^{2}$.

\subsubsection{The ${ }^{3} \mathrm{He}$ Detector}

The ${ }^{3} \mathrm{He}$ detector[17] was a cylindrical tube of $14.6 \mathrm{~cm}$ (length) x $3.8 \mathrm{~cm}$ (diameter) filled to a pressure of $8 \mathrm{~atm}$ with ${ }^{3} \mathrm{He}$. The energy dependence of the efficiency of the tube was estimated from the quoted pressure of ${ }^{3} \mathrm{He}$ and the assumption that neutrons incident on the detector have trajectories along the cords of the tube. Using the $2200 \mathrm{~m} \mathrm{~s}^{-1}$ cross section for the thermal group and $1 / \mathrm{E}$-weighted $1 / \mathrm{v}$ cross sections for the three lowest-energy groups in the Monte Carlo 
simulations, the fraction of incident neutrons in each group that undergo the ${ }^{3} \mathrm{He}(\mathrm{n}, \mathrm{p})$ reaction are given in Table 3.2.2.

Table 3.2.2. Group-averaged cross sections and the fractions of neutrons undergoing $(n, p)$ reactions in the ${ }^{3} \mathrm{He}$ tube assuming that neutron trajectories are normal to the tube axis.

\begin{tabular}{|c|c|c|c|c|}
\hline Group & Energy range $(\mathrm{MeV})$ & $\langle\square>(\mathrm{b})$ & $\square\left(\mathrm{cm}^{-1}\right)$ & $\left\langle\mathrm{f}_{\mathrm{np}}\right\rangle$ \\
\hline $1(\mathrm{th})$ & $0.0-4 \times 10^{-7}$ & 5317 & 1.144 & 0.6117 \\
\hline 2 & $4 \times 10^{-7}-1 \times 10^{-3}$ & 334 & $7.185 \times 10^{-2}$ & 0.1876 \\
\hline 3 & $1 \times 10^{-3}-0.1$ & 10.5 & $2.248 \times 10^{-3}$ & $6.703 \times 10^{-3}$ \\
\hline
\end{tabular}

\subsubsection{The Beam Monitor of the Generator Output}

The neutron generator's control system includes both source current and acceleration voltage. Uncertainties in reproducing these parameters along with variations in target characteristics and beam focusing generally combine to produce variable neutron output. In addition, the majority of experiments entail sequential repetitions of irradiation and counting periods and the time required for the generator output to achieve a quasi steady state is considerable (see below). As a result, it is necessary to monitor the generator output during each experiment.

The monitor is a small plastic detector mounted on a photomultiplier tube and has been shown to operate stably. The dependence of the monitor count rate on generator output was measured with the long counter suspended above and on-axis with the neutron beam at a source to detector distance of $8.2 \mathrm{~m}$. This is shown in Figure 3.2.3-1 when the high voltage of the generator was varied in the range $140-180 \mathrm{kV}$ and the source current varied in the range $0.25-4.0 \mathrm{~mA}$.

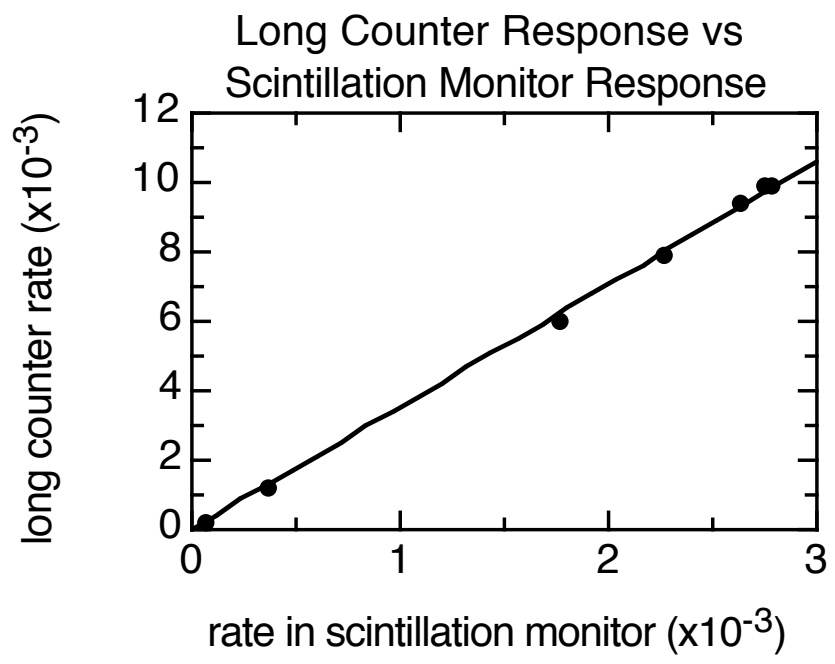

Figure 3.2.3-1. Variation in the long counter detection rate as a function of the detection rate in the beam monitor.

As seen, the monitor rate varies linearly with the long counter count rate. On the other hand, the rates in either counter did not vary linearly with source current above about $3 \mathrm{~mA}$ at an 
acceleration potential of $180 \mathrm{keV}$ (Figure 3.2.3-2). This is assumed to be due to changes in beam focus and location on the target when current becomes large.

Monitor Rate vs Source Current

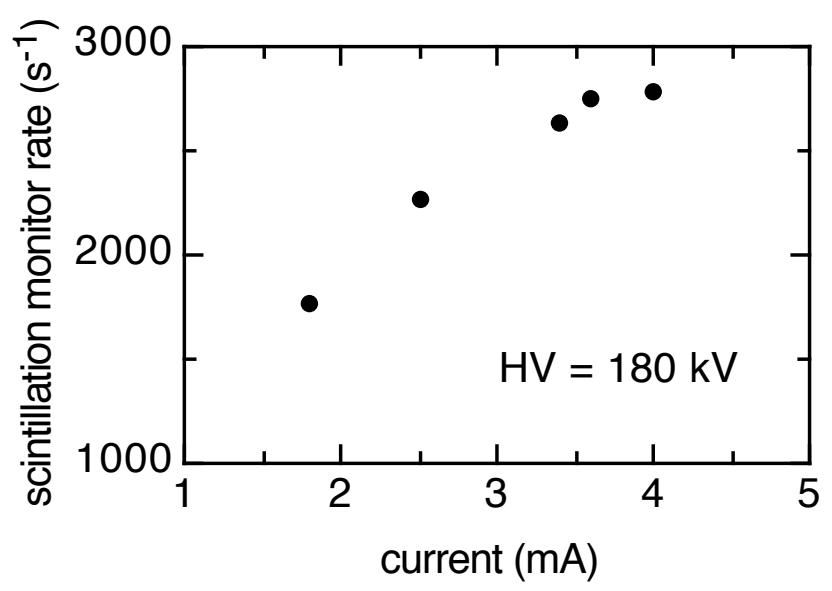

Figure 3.2.3-2. Beam monitor count rate vs. source current $(m A)$ at an acceleration voltage of $180 \mathrm{kV}$.

Taken together, the data in Figures 3.2.3-1 and 3.2.3-2 suggest that the count rate in the monitor is directly proportional to the generator output for source currents in the range $1-3 \mathrm{~mA}$ near the maximum operating potential. Based on these results, data from different experiments were normalized to the detection rate in the beam monitor.

\subsubsection{Reduction of personnel dose rates}

Monte Carlo computations allow simulation of radiation transport in complex 3D geometry. During the design of the experimental set-up, COG simulations helped define the source collimation and the type of shielding needed to reduce personnel radiation dose below levels required for safe operation in the cargo-scanning lab. To do so, COG simulates neutron and photon interactions in the various materials included in the model and accounts for moderation of the spectrum. The dose conversion data that converts simulated fluence to dose is taken from the ANSI/ASN standard 6.1.1 (1991).

The current baseline model that we use to simulate cargo interrogation experiments was first generated for the dose simulations. The model of the lab is detailed and includes the concrete walls, the concrete floor and the earth underneath, the roll-up doors, the pit with its stairs and landing platforms. It does not include the roof and personnel doors. The laboratory floor is empty of equipment or structures, unlike the "real" laboratory. The D-T generator is modeled as an isotropic source of $14.25 \mathrm{MeV}$ neutrons, located $93 \mathrm{~cm}$ below floor level in the NW corner of the pit, where the north and west walls offer intrinsic shielding. A description and a schematic of the collimator designed for the $14 \mathrm{MeV}$ neutron source is given earlier in Section 3.2.

Dose estimates for an open beam were obtained at 21 locations around the lab, 19 dose tallies were $1.52 \mathrm{~m}$ above the floor. Two tallies were $4.6 \mathrm{~m}$ above the floor on the East and West walls of the lab, since offices are located behind these walls on the second floor. The dose tallies are 
$22.9 \mathrm{~cm}$-diameter spheres, a close approximation of the geometry of the Remballs used for the dose surveys.

Simulations were compared to dose survey measurements and were in good agreement. Figure 3.2.4-1 shows the positions of the dose tallies (in black) and a comparison of dose survey measurements (in red) and dose estimates for a Radiation Generating Device (RDG) output of $1.0 \mathrm{e}+11$ neutrons/s. In general, the measured values were three times the model values when corrected for neutron output. This is consistent, after accounting for real world variables such as uncertainties on detector location, shielding blocks, instrument efficiencies, and location sensitivity close to the RDG.

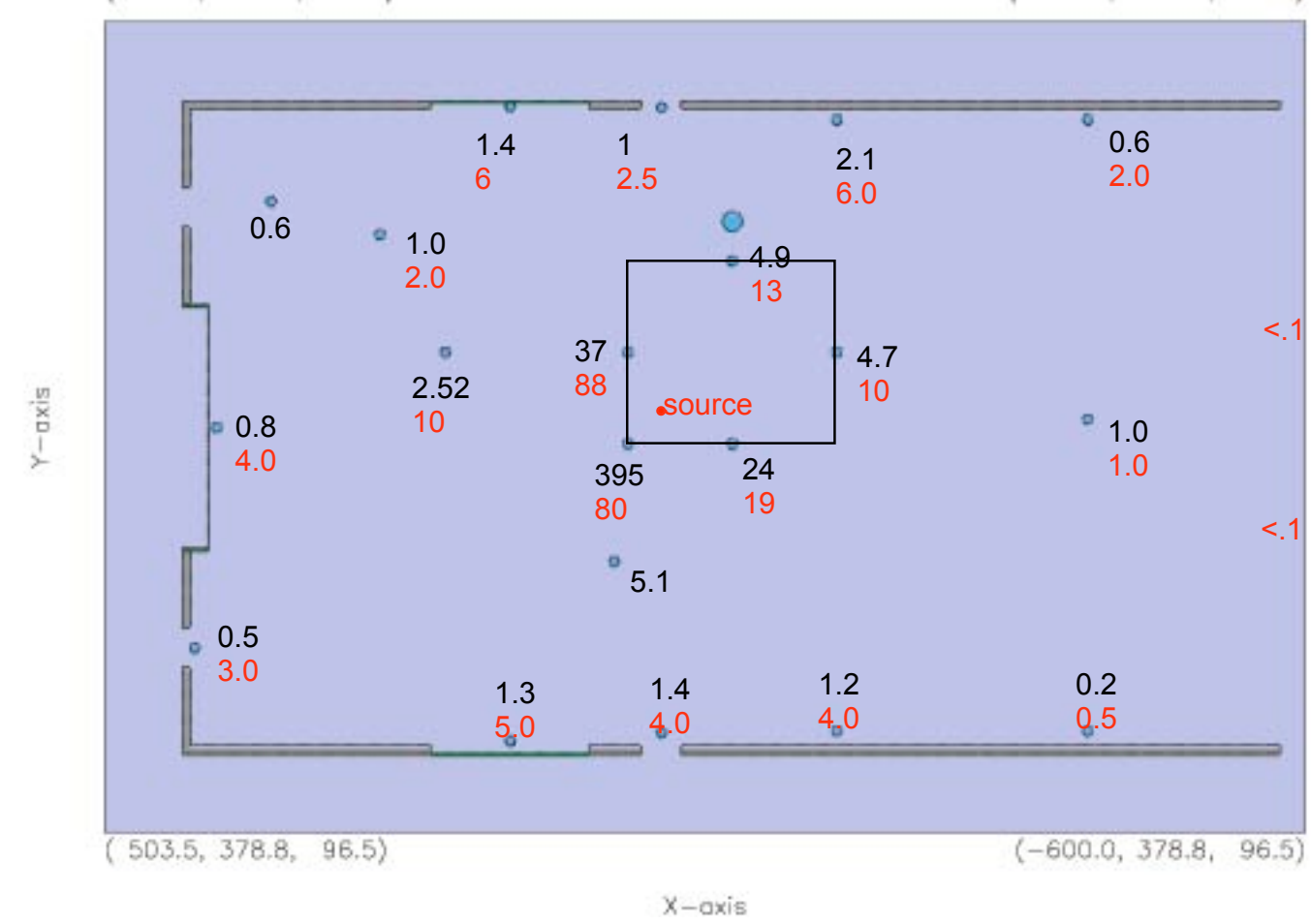

Figure 3.2.4-1 COG Dose estimates and Dose survey results at an elevation $z=1.52 \mathrm{~m}$ above the floor of the cargo scanning lab. Doses are in mrem $/ \mathrm{h}$ for an RDG output of 1.0e+11 neutrons/s. The grey outer boundary represents the wall of the lab. The blue disks show the location of the dose tallies, the black rectangle delineates the pit. Dose survey measurements are given in red and dose estimates in black.

\subsubsection{Shielding}

Dose estimates have been calculated based on the detailed model of the $7 \mathrm{MeV}$ D-D source described in Section 2.2.5 and below in Section 3.2.4.3. The collimator is a minor modification of the one designed for the $14.25 \mathrm{MeV}$ D-T generator described at the beginning of 3.2. We considered two cases, the open beam with no cargo present, and another scenario with surrogate steel cargo placed in the path of the beam. Compared to the open beam or the wood cargo, the steel cargo acts as a strong scatterer and weak absorber of neutrons. Most of the dose is due to neutrons initially in the collimated beam that scatter in the steel, causing the neutron dose 
around the experimental area to increase by a factor of 10X. These results are consistent with what we observed with the $14 \mathrm{MeV}$ source.

Several shielding configurations were simulated. The current design consists of two shadow shields placed on the east and west side of the source. They are made of polyethylene, and are $10.16 \mathrm{~cm}$ thick by $3.65 \mathrm{~m}$ high and $6 \mathrm{~m}$ long (the length of a small container). The shadow shields reduced dose estimates in the surrounding lab by a factor of $5 \mathrm{X}$ for the steel cargo. There was no additional dose reduction when adding a $10.6 \mathrm{~cm}$ - thick roof of polyethylene on top of these two walls.

\subsubsection{Collimation}

For an open beam or a cargo with neutron absorbing properties such as wood, simulations show that the external neutron dose around the experiment is determined by the neutron leakage through the collimator and thus by total neutron output and not by whether that output is isotropic. Although the maximum neutron energy will be lower, the new RFQ source has an output similar to the $14 \mathrm{MeV}$ generator. Accordingly, the collimator designed for the $14 \mathrm{MeV}$ neutron source will be used with the new RFQ source.

\subsubsection{Neutron target model}

The neutron source used in our initial cargo inspection experiments at LLNL was a simple (isotropic) $14.25 \mathrm{MeV}$ DT neutron generator that could easily be modeled using modern, general-purpose Monte Carlo radiation transport codes such as COG (LLNL) and MCNP or MCNPX (LANL); however, based on signal strength and induced background interference considerations, we have recently elected to develop and test a high-intensity, accelerator-driven, $\mathrm{D}(\mathrm{d}, \mathrm{n})^{3} \mathrm{He}$ source which should be capable of generating a neutron flux $\square \approx 7.7 \times 10^{5} \mathrm{n} / \mathrm{cm}^{2} / \mathrm{s}$ at the center of a cargo container (i.e. $\approx 15$ times that of our older DT source) with unscattered neutron energies in the range $\approx 3.5$ to $7.0 \mathrm{MeV}$. This should greatly enhance our experimental capabilities. However the strongly asymmetric angular distributions and kinematic correlations between neutron energy and emission angle characteristic of accelerator-driven, $\mathrm{D}(\mathrm{d}, \mathrm{n})^{3} \mathrm{He}$ sources make detailed analysis of the output complicated. Then too there is the gradual (but significant) degradation in incident $\mathrm{D}^{+}$ion energy associated with the extended geometry of the proposed source (a high-pressure $\mathrm{D}_{2}$ gas cell with a diameter of $\approx 5.08 \mathrm{~cm}$ and a total length of $\approx$ $40.1 \mathrm{~cm}$ ). These detailes presented major computational challenges since none of the aforementioned Monte Carlo codes are currently capable of modeling this level of detail using only their conventional (built-in) source structures. In order to work around this deficiency, we have taken advantage of a unique feature of LLNL's COG code - the ability to write specialized ("user-defined") source and detector packages that can be linked into the code at run time.

The COG user-defined source package ("USRSOR") that we have developed is capable of accurately modeling $\mathrm{D}(\mathrm{d}, \mathrm{n})^{3} \mathrm{He}$ nuclear reactions for any given distribution of deuterium atoms, whether diffused into a solid (e.g. deuterated Ti), bound up in a liquid (e.g. $\left.\mathrm{D}_{2} \mathrm{O}\right)$ or dispersed as a gas $\left(e . g . \mathrm{D}_{2}\right)$. The user must specify the names of a "source descriptor" file containing ordered pairs of $\mathrm{z}(\mathrm{cm})$ and KEdeut $(\mathrm{MeV})$ values (i.e. $\mathrm{D}^{+}$ion energy as a function of depth in the source medium) and two standard reference files containing $\mathrm{D}(\mathrm{d}, \mathrm{n})^{3} \mathrm{He}$ total cross section and angular distribution data derived from LLNL evaluated nuclear libraries (ENDL99) when calling this 
USRSOR and may (optionally) specify a number of modifying parameters. These include a source strength multiplier to scale COG detector tallies to match a given physical source, the $\mathrm{XYZ}$ translation of the center of the source distribution, the orientation of the source $\mathrm{Z}$ axis, a neutron emission "reference time" and/or beam pulse timing structure, the effective diameter of the incident $\mathrm{D}^{+}$ion beam (along with parameters which can be used to model the spatial profile of the beam as a "clipped" Gaussian if desired) and bias parameters which can be used to enhance statistical sampling of the angular distributions if needed. With this degree of flexibility, we can easily model the proposed $\mathrm{D}(\mathrm{d}, \mathrm{n})^{3} \mathrm{He}$ neutron source.

This source model has been successfully benchmarked against an array of analytical calculations and is now being used in simulations designed to characterize our new accelerator-driven, $\mathrm{D}(\mathrm{d}, \mathrm{n})^{3} \mathrm{He}$ source and determine radiation shielding requirements for laboratory personnel. Two examples are shown below. These include the predicted neutron flux and average neutron energy as a function of angle (Figure 3.2.4.3-1) and predicted neutron spectra at various elevations above source center (Figure 3.2.4.3-2).
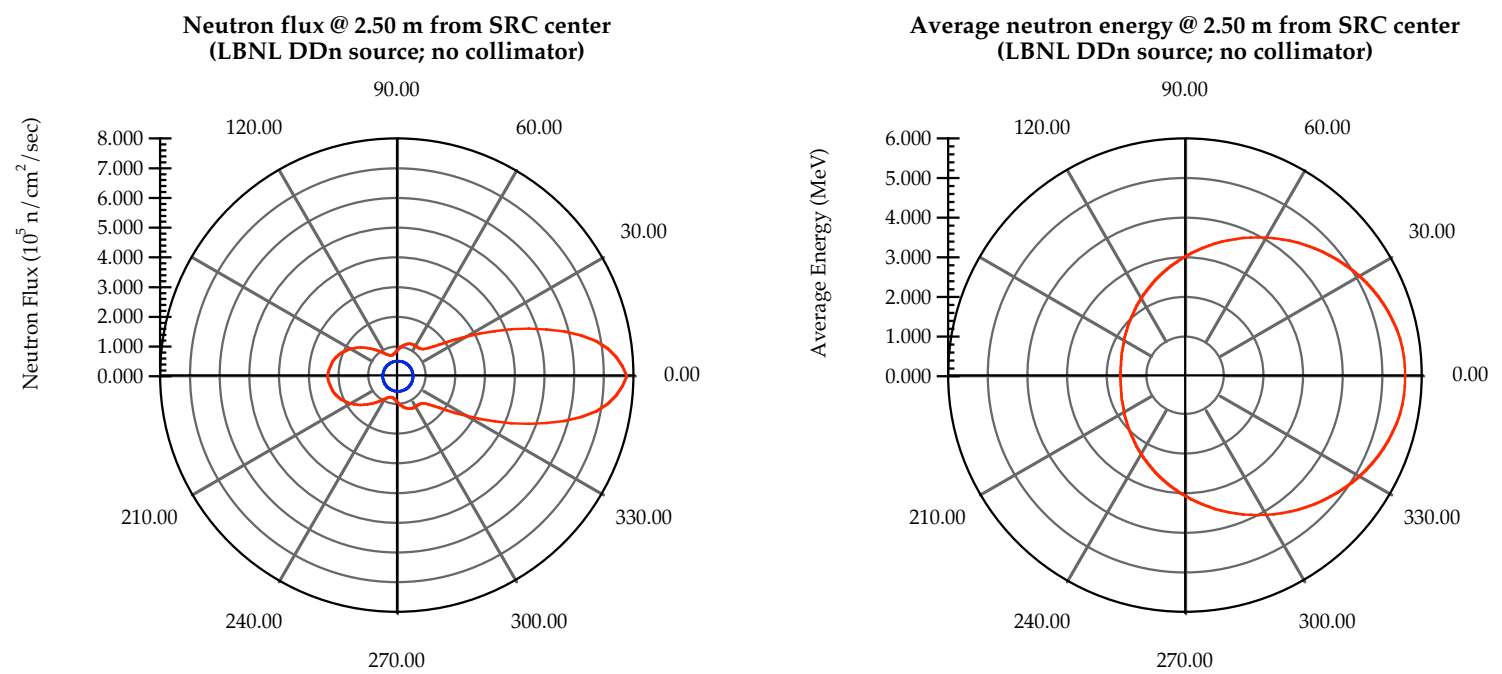

Figure 3.2.4.3-1. Predicted neutron flux $\left(\mathrm{n} / \mathrm{cm}^{2} / \mathrm{sec}\right)$ (left) and average neutron energy $(\mathrm{MeV})$ (right) at $2.50 \mathrm{~m}$ above source center for our proposed accelerator-driven $D(d, n)^{3}$ He neutron source. Flux values have been scaled to the predicted total yield of $\approx$ $1.12 \times 10^{11} \mathrm{n} / \mathrm{sec}$ into $4 \pi$ (for comparison, the flux of our older DT source at nominal output $\left(\approx 4 \times 10^{10} \mathrm{n} / \mathrm{sec}\right.$ into $\left.4 \pi\right)$ is indicated by the small blue circle on the left $)$. 

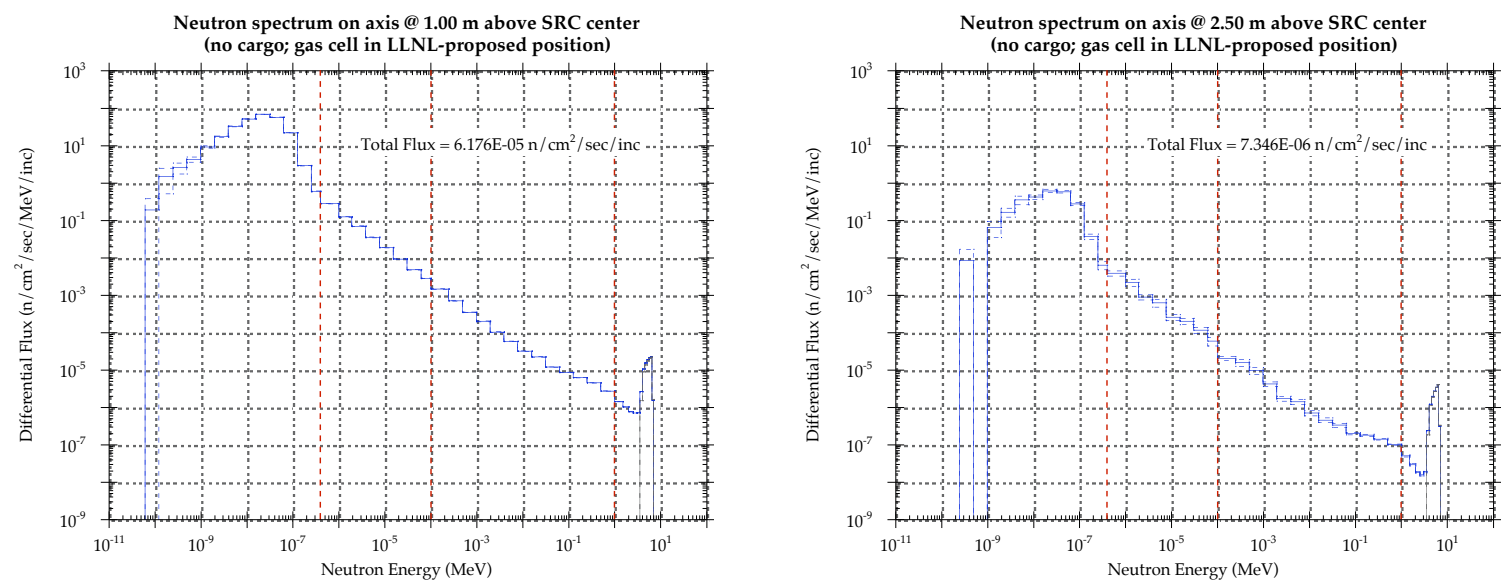

Figure 3.2.4.3-2. Predicted neutron spectra ( $\mathrm{n} / \mathrm{cm}^{2} / \mathrm{sec} / \mathrm{MeV} /$ source neutron) at $1.00 \mathrm{~m}$ (left) and $2.50 \mathrm{~m}$ (right) above source center for our proposed accelerator-driven $D(d, n)^{3} H e$ neutron source. The spectral intensity below $\approx 3.5 \mathrm{MeV}$ is due entirely to scattering in the source collimator structure and amounts to $\approx 30 \%$ of the total at $1.00 \mathrm{~m}$ (floor level) but only $\approx 6 \%$ of the total at $2.50 \mathrm{~m}$ (cargo level).

\subsubsection{Final assembly of the component testing lab}

The component testing lab has been assembled, revised, and assembled again over the past two years. It now contains a neutron source based on a $4 \mathrm{MeV}$ deuteron beam incident on a gas cell containing $\mathrm{D}_{2}$ gas. This beam is generated by the $\mathrm{D}(\mathrm{d}, \mathrm{n})$ reaction and produces a very intense of neutrons in the energy range 3-7 MeV and the beam intensity falls off dramatically at large angles, reducing the activation and dose burdens. Its details were given in Section 2.2.5. A full array of plastic detectors covering both sides of the cargo container is being installed to detect the Gray signature discussed earlier. There remains space for deployment of an array of moderated ${ }^{3} \mathrm{He}$ tubes to detect delayed neutrons as well. A modern data acquisition system acquires data in "event mode" so that all detector data can be re-sorted off-line to replay the experimental data for many variations in detector configuration. Several targets of HEU are available along with a variety of palletized cargo surrogates to simulate cargo attenuation. Finally, a translation system is being installed to move a $20 \mathrm{ft}$ container back and forth in front of the detector arrays and over the neutron source mounted in the floor. Details of these systems, except for the translation system, have been given elsewhere.

\subsubsection{Cargo translation system}

In order to replicate a "real life" scanning system, we need a realistic scanning type apparatus (i.e. trolley system) to mimic a "Nuclear Car Wash" configuration. We also need to study background variations as cargo and containers are moved into and out of the scanner area since those variations could trigger a false alarm (false positive) or a failure to detect (false negative). To that end, we designed a mechanical system that will translate a loaded cargo container past the neutron source, as would be the case in a deployed system. The system has been designed to 
translate a container from an initial "at-rest" position, move it past a neutron beam and interrogating detector array, and bring the container to a safe stop. The container can then be returned to its initial "start" position, and the process repeated. The nominal operating parameters for the system are as follows:

- Container: standard 6.1 m length

- Container speed: nominally $20 \mathrm{~cm} / \mathrm{s}$ (40 ft/min)

- Length of container travel: nominally $9.1 \mathrm{~m}$ total travel on $15 \mathrm{~m}$ long rails

- Operation: remote, from an operators station

- Positional feedback: encoder and video camera/monitor

- All operational procedures must adhere to ES\&H requirements

The translation design is comprised of three major subsystems: rail system, power transmission system, and container retrofit. It should be noted that this system is not intended to represent the field deployable version since, in this case, we are dealing with a floor over the neutron generator that cannot support the weight of a loaded container; and we also must address internal LLNL rules requiring substantial restraint for seismic safety so that an earthquake would not risk damage to the HEU samples that will be utilized in the experimental program.

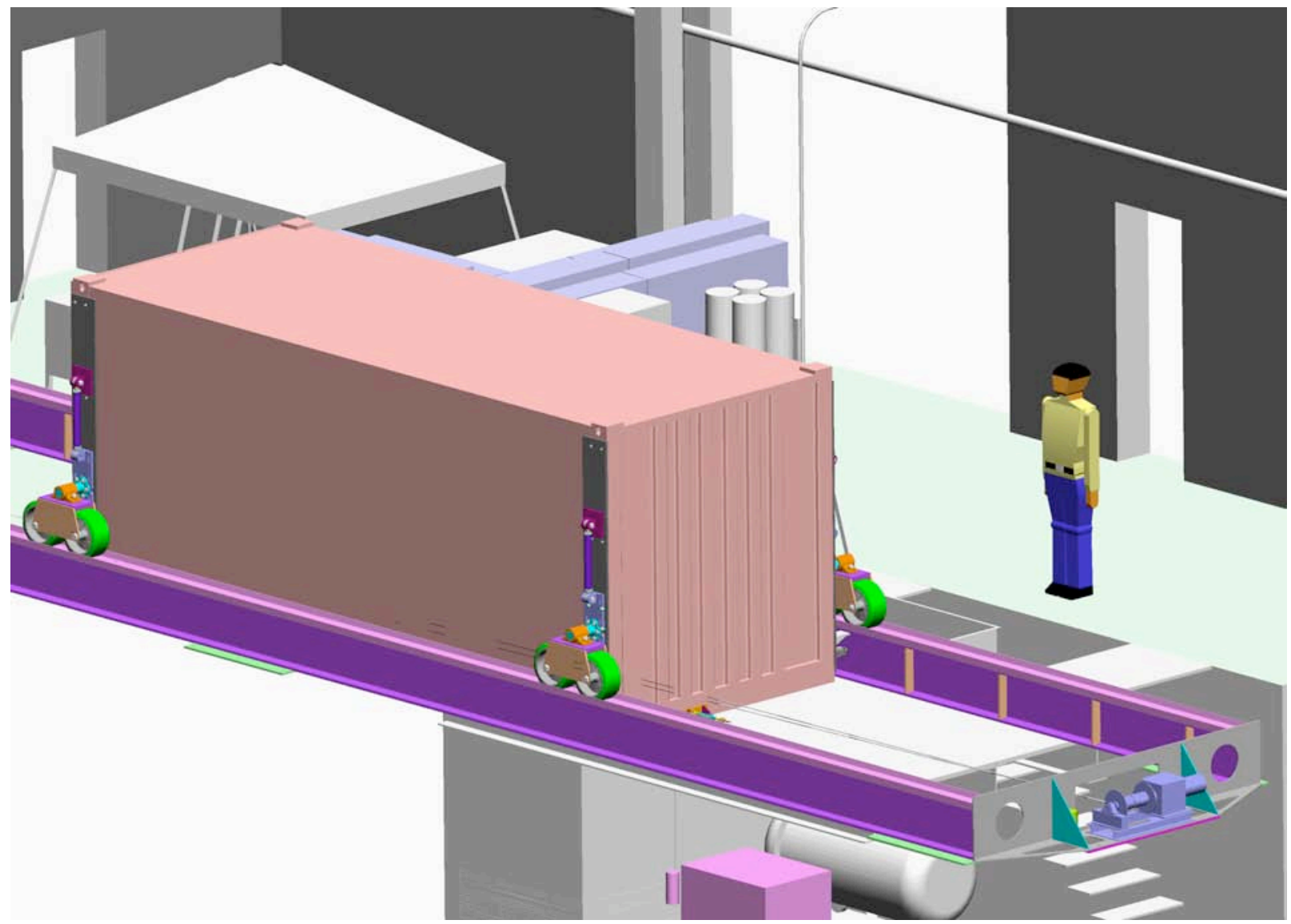

Figure 3.2.5.3-1 An overall "cut-away" view of the Cargo 
We designed a $15 \mathrm{~m}$ long rail system that incorporates reinforced W16 x 57 "I" beams and inverted C9 x 15 channels. The "I" beams support the weight and dynamics of the moving container, and the inverted channels that are welded to the top of the beams provide the track configuration that the container wheels ride in. On both ends of the track we have incorporated ramps, which act as redundant stopping devices. The unit also has gusseted floor plates allowing us to bolt the rails to the concrete floor. The entire unit is made of steel, and is of welded construction.

We designed a power transmission system that translates a $6.1 \mathrm{~m}$ cargo container from a standstill position to a stopping position $9.1 \mathrm{~m}$ away. Our plan employs an industry standard single winch that is both over-wound and under-wound with a cable. In effect, this single winch produces a push or pull action which allows the container to be moved in either direction. The winch is remotely operated from the operator's station, and is equipped with many features to insure safe operation. This system can move the fully loaded container slowly or at speeds up to $60 \mathrm{~cm} / \mathrm{s}$ and bring it to a graceful stop at the end of the track. The maximum speed corresponds to completing the scan of a $40 \mathrm{ft}$ container in $60 \mathrm{~s}$.

We will be retrofitting our existing $6.1 \mathrm{~m}$ cargo container, adapting it to be conveyed on the newly designed rail system. The vertical corner structure of any given container is the strongest structural member; to each of these corners we will attach mounting plates, and we will fasten to these plates an adjustable tandem-wheel assembly. Although the wheels and bearings are standard components that can be purchased "off-the-shelf", the assemblies themselves and associated hardware have been custom designed to meet very stringent requirements. The container retrofit includes container attachment devices for integration with the power transmission system.

\subsection{Comparing Long Counter and ${ }^{3} \mathrm{He}$ tube data with the Baseline Model Monte Carlo Simulations.}

One of the principal goals of the program described in this document is the validation of the simulation tool COG. For this purpose a variety of experiments have been conducted utilizing the $14 \mathrm{MeV}$ neutron source. These included measurements with no HEU present and measurements with HEU embedded in surrogate cargos of wood and steel at appropriate densities. Detailed measurements of the fission product signals and of the beam characteristics were carried out for comparison with model predictions. The methodology and results are described below.

Experimental measurements on neutron detection rates were performed with both the long counter and the ${ }^{3} \mathrm{He}$ tube $[18,19]$. The long counter was suspended from a crane along the axis of the neutron beam at floor-to-detector face distances in the range $48-140 \mathrm{~cm}$. The ${ }^{3} \mathrm{He}$ tube was also located axially in the same range of heights as well as at five locations located at $91 \mathrm{~cm}$ normal to the collimator axis. In all measurements the axis of the tube was in the horizontal dimension.

A Monte Carlo model[20] was developed using the baseline model[15] and the experimental arrangement shown in Figure 3.3-1. The model included the pit and its structures, the collimator, the concrete floors and a thick layer of soil underneath the area directly around the pit and the 
steel cover plate of the pit. It did not include the building walls or other structures in the laboratory. The $(\mathrm{d}, \mathrm{t})$ target was modeled as a cylinder of $3.2 \mathrm{~mm}$ (radius) $\times 2.54 \mathrm{~cm}$ (height) centered at the geometric origin of the model. The center of the source is located $93 \mathrm{~cm}$ below the steel cover plate of the pit. The source was assumed to be isotropic and monoenergetic at an energy of $E_{n}=14.25 \mathrm{MeV}$.
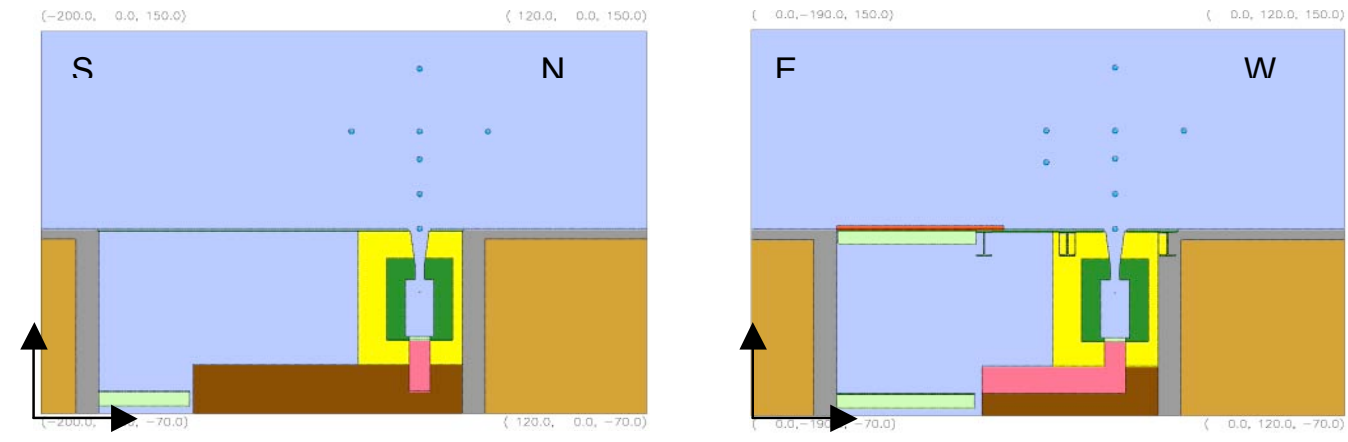

Figure 3.3-1 . Schematic of the Monte Carlo model used in the calibration of the long counter (see Figure 3.2-1). The blue dots represent the locations of boundary-crossing detectors to tally the total neutron fluence and the fluences in 7 energy bins (see text).

Neutron fluences per source particle were obtained by locating 7-cm (diameter) spherical surfaces centered at the five locations along the beam axis and 5 locations off-axis shown in Figure 3.3-1. The fluence was considered in the seven energy ranges $0-4.0 \times 10^{-7} \mathrm{MeV}, 4.0 \times 10^{-7}$ - $1.0 \times 10^{-3} \mathrm{MeV}, 1.0 \times 10^{-3}-0.1 \mathrm{MeV}, 0.1-1.0 \mathrm{MeV}, 1.0-5.0 \mathrm{MeV}, 2.0-14.2 \mathrm{MeV}$ and 1.42 $1.43 \mathrm{MeV}$, the first range considered to be thermal neutrons and the last range considered to represent the uncollided flux from the source.

\subsubsection{Relative Axial Variation of the Fast Flux due to $14 \mathrm{MeV}$ source}

Spatial variation in neutron intensity strongly effects the flux that produces the fission signal characteristic of SNM. Those variations have been quantified both with predictions and confirming measurements.

The uncollided flux from the $14 \mathrm{MeV}$ generator is expected to be isotropic to an excellent approximation. The simulations were shown to reproduce the expected $1 / \mathrm{r}^{2}$ variation within statistical errors as demonstrated in Table 3.3.1-1. 
Table 3.3.1-1: Simulated uncollided neutron fluences from the Monte Carlo baseline model calculations. The columns represent, respectively, the distance of the face of long counter from laboratory floor, the radial dimension between source and the effective center of the long counter center, the simulated fluence of uncollided neutrons, the fluences normalized to the fluence at $d=140 \mathrm{~cm}$ and the normalized values of $1 / r^{2}$. The errors shown represent estimated statistical errors only.

\begin{tabular}{|c|c|c|c|l|}
\hline $\mathrm{d}(\mathrm{cm})$ & $\mathrm{r}(\mathrm{cm})$ & $\square_{\text {sim }}\left(\mathrm{n} \mathrm{cm}^{-2} \mathrm{sn}^{-1}\right)$ & $\square(\mathrm{r}) / \square_{140}$ & $r_{140}^{2} / r^{2}$ \\
\hline 0 & 92.7 & $(9.17 \pm 0.09) \times 10^{-6}$ & $6.31 \pm 0.12$ & 6.28 \\
\hline 48 & 141.0 & $(3.97 \pm 0.04) \times 10^{-6}$ & $2.73 \pm 0.05$ & 2.72 \\
\hline 94 & 186.7 & $(2.20 \pm 0.02) \times 10^{-6}$ & $1.51 \pm 0.03$ & 1.55 \\
\hline 140 & 232.4 & $(1.45 \pm 0.02) \times 10^{-6}$ & $1.00 \pm 0.02$ & 1.00 \\
\hline 230 & 322.6 & $(7.29 \pm 0.13) \times 10^{-7}$ & $0.50 \pm 0.01$ & 0.52 \\
\hline
\end{tabular}

In the absence of direct measurements, the spectral distributions from the Monte Carlo simulations were used to estimate the contribution of all energy groups with $E_{n}>1 \mathrm{keV}$ to the long counter detection rate. The parameterization of the relative efficiency function discussed above was used to estimate the mean relative efficiency of the five groups as shown in Table 3.3.1-2.

Table 3.3.1-2: Long counter relative efficiencies and effective areas (sensitivities) for the five neutron groups contributing to the detection rate. The relative efficiencies given in the second column represent the mean efficiencies calculated with the correlation from Figure 3.2.1-2 at the upper and lower boundary of each energy group.

\begin{tabular}{|l|c|c|c|}
\hline Energy bin & $\begin{array}{l}\text { Relative efficiency } \\
\text { (from fit) }\end{array}$ & $\begin{array}{l}\text { Relative efficiency } \\
\text { normalized at 2.3MeV }\end{array}$ & $\begin{array}{l}\text { Effective area } \\
\left(\mathrm{cm}^{2}\right)\end{array}$ \\
\hline $0.001-0.1$ & $1.103 \pm 0.048$ & $1.071 \pm 0.057$ & $3.694 \pm 0.151$ \\
\hline $0.1-1.0$ & $1.086 \pm 0.046$ & $1.055 \pm 0.055$ & $3.638 \pm 0.150$ \\
\hline $1.0-5.0$ & $1.009 \pm 0.038$ & $0.979 \pm 0.049$ & $3.378 \pm 0.148$ \\
\hline $5.0-14.2499$ & $0.826 \pm 0.024$ & $0.802 \pm 0.039$ & $2.768 \pm 0.145$ \\
\hline $14.2499-14.25$ & $0.715 \pm 0.014$ & $0.694 \pm 0.033$ & $2.396 \pm 0.144$ \\
\hline
\end{tabular}

The relative sensitivities were combined with the simulated group fluences to obtain the predicted relative detection rates in the long counter shown in Table 3.3.1-3 along with the experimental detection rates. The detection rates, relative to the respective values at $140 \mathrm{~cm}$ are in remarkably good agreement. We take these results to indicate that the simulations with the baseline model represent, to a reasonable degree, the spectral distributions of neutrons with energies $E_{n}=2 \mathrm{keV}$ to $15 \mathrm{MeV}$ emanating from the collimator at distances of $1.4-3.2 \mathrm{~m}$ above the neutron source. 
Table 3.3.1-3. Relative detection rates from the Monte Carlo simulations compared to experimental measurements with the long counter. The second column gives the simulated detected fluence and the third column gives the experimental count rate. The fourth and fifth columns give the simulated and experimental detection rates relative to the rate at $140 \mathrm{~cm}$ above the floor.

\begin{tabular}{|c|c|c|c|c|}
\hline $\mathrm{d}(\mathrm{cm})$ & $\square \square_{i} A_{\text {eff }}\left(\mathrm{sn}^{-1}\right)$ & $\mathrm{R}_{\exp }\left(\mathrm{ct} \mathrm{cm}^{-2} \mathrm{~s}^{-1}\right)$ & $\square \square_{i} A_{\text {eff }} /\left(\square \square_{i} A_{\text {eff }, i}\right) 140\left(\mathrm{sn}^{-1}\right)$ & $\mathrm{R}_{\text {calc }} / \mathrm{R}_{140, \text { calc }}$ \\
\hline 0.0 & $(4.48 \pm 0.15) \times 10^{-5}$ & & $10.2 \pm 0.12$ & \\
\hline 48 & $(1.37 \pm 0.06) \times 10^{-5}$ & $3522 \pm 5$ & $3.13 \pm 0.05$ & $3.17 \pm 0.01$ \\
\hline 94 & $(7.09 \pm 0.33) \times 10^{-6}$ & & $1.62 \pm 0.03$ & \\
\hline 140 & $(4.38 \pm 0.22) \times 10^{-6}$ & $1112 \pm 3$ & $1.00 \pm 0.02$ & $1.00 \pm 0.06$ \\
\hline 230 & $(2.15 \pm 0.11) \times 10^{-6}$ & $551 \pm 2$ & $0.49 \pm 0.01$ & $0.496 \pm 0.005$ \\
\hline
\end{tabular}

\subsubsection{Relative Axial Variation of the Low-energy Neutron Flux}

With the group-averaged efficiencies from Table 3.3.1-1, the simulated detection rates in the ${ }^{3} \mathrm{He}$ tube are given in Table 3.3.2-1 and are compared with experimental measurements in Table 3.3.2-2. Notwithstanding the neglect of neutron scattering in the structures of the laboratory hall, the relative detection rates are in agreement with experiment within $\pm 2 \square$.

Table 3.3.2-1. Simulated detection rates in the ${ }^{3}$ He tube from the COG calculations. Subscripts represent energy groups considered beginning with the thermal group (see text). Estimated errors $( \pm 1 \square)$ in the total detection rates include statistical errors in the COG simulations and an estimated $8 \%$ error in the average detection probabilities.

\begin{tabular}{|c|c|c|c|c|c|c|c|}
\hline $\mathrm{d}(\mathrm{cm})$ & $\begin{array}{c}\square_{1} \\
\left(\mathrm{~cm}^{-2} \mathrm{~s}^{-1}\right)\end{array}$ & $\begin{array}{c}<\mathrm{f}_{\mathrm{np}}>\square_{1} \\
\left(\mathrm{~cm}^{-2} \mathrm{~s}^{-1}\right)\end{array}$ & $\begin{array}{c}\square_{2} \\
\left(\mathrm{~cm}^{-2} \mathrm{~s}^{-1}\right)\end{array}$ & $\begin{array}{c}<\mathrm{f}_{\mathrm{np}}>\square_{2} \\
\left(\mathrm{~cm}^{-2} \mathrm{~s}^{-1}\right)\end{array}$ & $\begin{array}{c}\square_{3} \\
\left(\mathrm{~cm}^{-2} \mathrm{~s}^{-1}\right)\end{array}$ & $\begin{array}{c}<\mathrm{f}_{\mathrm{np}}>\square_{3} \\
\left(\mathrm{~cm}^{-2} \mathrm{~s}^{-1}\right)\end{array}$ & $\square_{1}^{3} \square_{i}\left\langle f_{n p}\right\rangle_{i}$ \\
\hline 0 & $4.69(-6)$ & $2.86(-6)$ & $1.42(-6)$ & $2.66(-7)$ & $1.90(-6)$ & $1.27(-8)$ & $3.14 \pm 0.23(-6)$ \\
\hline 48 & $3.25(-7)$ & $2.00(-7)$ & $1.62(-7)$ & $3.04(-8)$ & $2.32(-7)$ & $1.55(-9)$ & $2.31 \pm 0.17(-7)$ \\
\hline 99 & $1.01(-7)$ & $6.15(-8)$ & $5.05(-8)$ & $9.47(-9)$ & $1.01(-7)$ & $6.77(-10)$ & $7.17 \pm 0.58(-8)$ \\
\hline 140 & $4.90(-8)$ & $3.00(-8)$ & $2.59(-8)$ & $4.86(-9)$ & $4.23(-8)$ & $2.83(-10)$ & $3.51 \pm 0.35(-8)$ \\
\hline 230 & $1.56(-8)$ & $9.57(-9)$ & $1.01(-8)$ & $1.90(-9)$ & $2.01(-8)$ & $1.35(-10)$ & $1.16 \pm 0.12(-8)$ \\
\hline
\end{tabular}


Table 3.3.2-2. Comparison of experimental relative low-energy detection rates with those calculated from the baseline model. The errors in the experimental rates are taken to be $2 \%( \pm \square)$.

\begin{tabular}{|c|c|c|c|}
\hline $\begin{array}{c}\mathrm{d} \\
(\mathrm{cm})\end{array}$ & $\mathrm{R}_{\mathrm{exp}}\left(\operatorname{cts~s}^{-1}\right)$ & $R_{\text {exp }}^{\dagger}$ & $\bigcap_{1}^{3} a_{i}\left\langle f_{n p}\right\rangle_{i}$ \\
\hline 0 & - & - & $89.5 \pm 11.1$ \\
\hline 48 & $3249 \pm 65$ & $6.02 \pm 0.17$ & $6.58 \pm 0.81$ \\
\hline 99 & $1090 \pm 22$ & $2.02 \pm 0.06$ & $2.04 \pm 0.26$ \\
\hline 140 & $540 \pm 11$ & 1.00 & 1.00 \\
\hline 230 & $232 \pm 5$ & $0.43 \pm 0.01$ & 05 \\
\hline
\end{tabular}

This data shows excellent agreement between measured neutron fluxes at several positions and those predicted by the enhanced COG code. This constitutes an important part of the validation process for this essential tool.

\subsubsection{Relative Horizontal Variation of the Flux of Low-energy Neutrons}

As in the preceding section it essential to accurately measure neutron fluxes away from the beam axis and compare those measurements with COG simulations as part of the code validation process. The relative experimental detection rates in the ${ }^{3} \mathrm{He}$ tube at the five off-axis locations are compared with the Monte Carlo predictions in Table 3.3.3-1. We have used the same estimates of the fractional group detection probabilities as described above. Again the relative detection rates are in agreement with one another within $\pm 2 \square$. We interpret these to indicate that the neglect of some details of the experimental arrangements and the physical characteristics of the experimental hall are fairly unimportant in determining the low-energy neutron flux within a few meters normal to the opening of the collimator throat.

Table 3.3.3-1. Comparison of the relative simulated and experimental detection rates in the ${ }^{3} \mathrm{He}$ tube at 5 positions located $91 \mathrm{~cm}$ normal to the axis of the neutron beam. The columns give, respectively, the location of the detector $(\mathrm{cm})$, the simulated total detection rate, the experimental detection rate, the simulated and experimental detection rates normalized to the rates on axis at a height of $140 \mathrm{~cm}$ above the laboratory floor and the ratio of simulated to experimental rates.

\begin{tabular}{|c|c|c|c|c|c|}
\hline $\begin{array}{c}\text { Location } \\
(\mathrm{x}, \mathrm{y}, \mathrm{z})(\mathrm{cm})\end{array}$ & $\square_{1}^{3} \square_{i}\left\langle f_{n p}\right\rangle_{i}$ & $\begin{array}{l}\mathrm{R}_{\mathrm{exp}} \\
\left(\mathrm{s}^{-1}\right)\end{array}$ & $\bigcap^{3} \square_{i}\left\langle f_{n p}\right\rangle_{i}$ & $\begin{array}{l}R_{\exp }^{\dagger} \\
\left(\mathrm{s}^{-1}\right)\end{array}$ & $\left.{ }_{n p}\right\rangle_{i} \stackrel{\square}{\mathscr{H}} / R_{\exp }^{\dagger}$ \\
\hline 0, E91, 140 & $2.10 \pm 0.24(-8)$ & $293 \pm 6$ & $0.598 \pm 0.091$ & $0.542 \pm 0.016$ & $1.10 \pm 0.17$ \\
\hline S91, 0, 140 & $1.94 \pm 0.20(-8)$ & $237 \pm 5$ & $0.553 \pm 0.079$ & $0.439 \pm 0.013$ & $1.26 \pm 0.18$ \\
\hline $0, \mathrm{~W} 91,140$ & $1.93 \pm 0.20(-8)$ & $263 \pm 5$ & $0.550 \pm 0.079$ & $0.487 \pm 0.014$ & $1.13 \pm 0.16$ \\
\hline $\mathrm{N} 91,0,140$ & $2.03 \pm 0.23(-8)$ & $258 \pm 5$ & $0.578 \pm 0.087$ & $0.478 \pm 0.013$ & $1.21 \pm 0.18$ \\
\hline S91, 0, 94 & $2.00 \pm 0.22(-8)$ & $223 \pm 4$ & $0.570 \pm 0.085$ & $0.413 \pm 0.011$ & $1.38 \pm 0.21$ \\
\hline
\end{tabular}




\subsubsection{Absolute Calibration of the Generator output}

The absolute output of the neutron generator can be estimated from the data given in Table 3.3.4-1 and the fraction of the detection rate due to uncollided neutrons estimated from the Monte Carlo simulations.

Table 3.3.4-1. Estimated detection rates of uncollided source neutrons and the corresponding neutron source strengths for the experimental data given in Table 3.3.1-2. The second column is the distance from the target center to the effective center of the long counter estimated for 14.25-MeV neutrons $(23.5 \mathrm{~cm})$, the fourth column gives the predicted fractions of the detected rates due to uncollided neutrons, and the last column gives the estimated generator output.

\begin{tabular}{|c|c|c|c|c|c|}
\hline $\begin{array}{c}\mathrm{d} \\
(\mathrm{cm})\end{array}$ & $\begin{array}{c}\mathrm{d}+1 \\
(\mathrm{~cm})\end{array}$ & $\begin{array}{c}\mathrm{R}_{\text {exp }} \\
\left(\mathrm{ct} \mathrm{cm}^{-2} \mathrm{~s}^{-1}\right)\end{array}$ & $\square_{14} A_{\text {eff,14 }} / \square \square_{i} A_{\text {eff }, i}$ & $\begin{array}{c}\mathrm{R}_{14} \\
\left(\mathrm{ct} \mathrm{cm}^{-2} \mathrm{~s}^{-1}\right)\end{array}$ & $\begin{array}{c}\mathrm{S}_{14} \\
\left(\mathrm{n} \mathrm{s}^{-1}\right)\end{array}$ \\
\hline 48 & 164.5 & $3522 \pm 5$ & $0.693 \pm 0.051$ & $2441 \pm 180$ & $3.46 \pm 0.42 \times 10^{8}$ \\
\hline 140 & 255.9 & $1112 \pm 3$ & $0.795 \pm 0.063$ & $884 \pm 72$ & $3.04 \pm 0.39 \times 10^{8}$ \\
\hline 230 & 346.0 & $551 \pm 2$ & $0.811 \pm 0.066$ & $447 \pm 36$ & $2.81 \pm 0.36 \times 10^{8}$ \\
\hline
\end{tabular}

The experimental data were accumulated at a nominal accelerating potential and source current of $100 \mathrm{kV}$ and $1.55 \mathrm{~mA}$, respectively. To the extent that these voltages and currents are constant and reproducible, the data in Table 3.3.4-1 establish an absolute calibration of the generator at one set of operating parameters. Note that the derived source strengths are identical within experimental errors of about $12-13 \%$ where the error is dominated by the uncertainty in the effective efficiency function of the long counter and the uncertainty in the fraction of detected events ascribed to the uncollided flux.

Experience with operation of the neutron generator has shown that the limit of stable operation is found at an acceleration voltage of $190 \mathrm{kV}$ and a source current of about $3.75 \mathrm{~mA}$. Under such conditions, the rate in the beam monitor is typically $2.8 \times 10^{3} \mathrm{~s}^{-1}$ and thus the maximum source strength is about $4 \times 10^{10} \mathrm{~s}^{-1}$.

The data in Table 3.3.4-1 are now used to derive the absolute source strength per unit count rate in the beam monitor as shown in Table 3.3.4-2. Again, the source strengths are in agreement with one another within $\pm 1 \square$.

Table 3.3.4-2. Generator source strength per unit count rate in the beam monitor at the nominal accelerating potential and source current of $100 \mathrm{kV}$ and $1.55 \mathrm{ma}$, respectively.

\begin{tabular}{|c|c|c|c|c|c|}
\hline $\begin{array}{c}\mathrm{d} \\
(\mathrm{cm})\end{array}$ & $\begin{array}{c}\mathrm{S} \\
\left(\mathrm{n} \mathrm{s}^{-1}\right)\end{array}$ & Monitor counts & $\begin{array}{c}\text { Live time } \\
\left(\mathrm{s}^{-1}\right)\end{array}$ & $\begin{array}{c}\mathrm{R}_{\mathrm{m}} \\
\left(\mathrm{s}^{-1}\right)\end{array}$ & $\mathrm{S}_{14} / \mathrm{R}_{\mathrm{m}}$ \\
\hline 48 & $3.46 \pm 0.42 \times 10^{8}$ & 2791 & 125.7 & $22.20 \pm 0.19$ & $1.56 \pm 0.19 \times 10^{7}$ \\
\hline 140 & $3.04 \pm 0.39 \times 10^{8}$ & 3572 & 173.6 & $20.58 \pm 0.34$ & $1.48 \pm 0.19 \times 10^{7}$ \\
\hline 230 & $2.81 \pm 0.36 \times 10^{8}$ & 2822 & 131.1 & $21.53 \pm 0.40$ & $1.30 \pm 0.17 \times 10^{7}$ \\
\hline
\end{tabular}




\subsection{Comparison of the Baseline Model with the Number of Fissions Induced in a HEU Target with and without Idealized Cargos}

The ability of the baseline model to predict the number of fissions and fission rates induced in a HEU target was tested by irradiation of the target in the absence of other materials - "bare" target irradiations - and in the presence of simulated cargos composed of commercial plywood and an array of steel pipes designed to provide an approximation to low-density higher-Z materials. In each experiment the target was centered on the beam axis and was subjected to a series of identical irradiation/counting cycles. During the counting interval, the photons emitted from the assembly were detected with liquid scintillation counters and/or an array of solid plastic scintillators. The nominal irradiation and decay periods per cycle were $30 \mathrm{~s}$ and $100 \mathrm{~s}$, respectively. The neutron generator output and the counting period were controlled automatically.

Following the total irradiation/counting period, the number of fissions induced in the target was determined by assay of the principal $\square$ rays emitted in decay of the ${ }^{91,92} \mathrm{Sr}$ fission products with an HPGe spectrometer. The Sr isotopes were chosen because of their large fission yields, welldefined decay schemes and convenient half-lives.

In the limit that the HEU target is subjected to $\mathrm{N}$ identical irradiation/counting cycles, each consisting of an irradiation period of length $\square$ and a decay period of length ( $\mathrm{T}-\square$, and each at the same average neutron source strength, the number of atoms of a fission product remaining at the time $\mathrm{t}=\mathrm{NT}$ is

$$
n(N T)=\frac{r}{\square}\left(1 \square e^{\square[\square}\right) e^{\square(C T \square \square)} \frac{\left(1 \square e^{\square[N T}\right)}{1 \square e^{\square \square T}}=f r .
$$

Here $\mathrm{r}$ is the rate of production of the fission product and is given by

$$
r=n_{T} \square \square_{f}(E) y(E) \square(E) d E
$$

where $\square_{f}(E), y(E)$ and $\square(\square)$ are the fission cross-section, the fission yield of the nuclide in question and the neutron flux, respectively.

The average value of the fission yield over the neutron spectrum is

$$
\langle y(E)\rangle=\frac{\square \square_{f}(E) y(E) \square(E) d E}{\square \square_{f}(E) \square(E) d E} .
$$

The fission rate, $\mathrm{F}$, is given by

$$
F=n_{T} \square \square_{f}(E) \square(E) d E
$$

and thus the rate of production can be written 


$$
r=n_{t}\langle y(E)\rangle \square \square_{f}(E) \square(E) d E=F\langle y(E)\rangle .
$$

The number of atoms of a fission product at the end of the total irradiation period is then

$$
n(N T)=F\langle y(E)\rangle .
$$

As shown in Eq. 3.4-1 through 3.4-5 the number of fissions produced depends on the details of the irradiation, i.e. neutron intensity, energy spectrum, irradiation timing, and number of repetitions. On the other hand measurement of the number of fissions produced for comparison with simulation results depends only on measurement of the radioactivity due to a few wellunderstood fission products and their yield in fission. The fission product yield per fission is independent of neutron intensity or the timing of the irradiations. Also, given that thermal neutron induced fission dominates over fast fission in all of cases of interest here, the fission yield is nearly independent of neutron energy spectrum as well. In the limit that the energy dependence of the fission yield is unimportant, the estimation of $\mathrm{n}(\mathrm{NT})$ by determining the number of atoms of a fission product present at the end of the total irradiation/decay sequence from Eq. 3.4-6 is completely independent of the details of the irradiation scheme, including the neutron spectrum and intensity. Its error is defined primarily by the uncertainties in the photopeak intensity of the nuclide of interest extracted from the measured $\square$-ray spectrum; the decay scheme and fission yield of the isotope and the absolute efficiency of the HPGe. In practice, the fission yields of ${ }^{91,92} \mathrm{Sr}$ are somewhat energy dependent and an additional small error will be incurred by neglect of this effect.

This estimate can be compared with the value of n(NT) estimated from the Monte Carlo simulations by use of the fission yield averaged over the simulated neutron spectrum, the simulated total fission rate per source neutron and the experimental estimate of the strength of the neutron source given by the correlation between the source strength and the count rate in the beam monitor. This estimate is clearly independent of the decay scheme of the nuclide in question and the intensity measurements made with use of the HPGe.

\subsubsection{The HEU Target}

The HEU target used in all initial experiments was composed of $\mathrm{U}_{3} \mathrm{O}_{8}$ double encapsulated in thin steel cans. The oxide was produced by high temperature oxidation of the metal in situ and was not processed further. The oxide so formed has an average density much smaller than the theoretical value and there is good reason to believe that the density is not uniform throughout. The target has not been subject to $\mathrm{x}$-ray examination to determine its exact macroscopic structure.

Based on the specifications of the HEU and its container, the target was modeled with the following characteristics.

$$
\begin{array}{ll}
\text { density } & -2.4 \mathrm{~g} \mathrm{~cm}^{-3} \\
\text { diameter } & -8.64 \mathrm{~cm} \\
\text { mean height } & -3.34 \mathrm{~cm} .
\end{array}
$$




$$
\begin{array}{ll}
\text { enrichment } & -94.71 \% \text { in }{ }^{235} \mathrm{U} \\
\text { can walls } & - \text { steel } \\
\text { wall thickness }-1.0 \mathrm{~mm} \\
\text { double-encapsulated }
\end{array}
$$

\subsubsection{Actual Irradiation Periods}

The time profile of the irradiation and counting periods during sequential irradiations and the gating of the data acquisition system for counting in between irradiations was provided by specially built control box. The nominal total and irradiation times of a single period are $\mathrm{T}=130$ $\mathrm{s}$ and $\square=30 \mathrm{~s}$, respectively. However, because of the times required for the generator high voltage and focusing to reach steady state, the actual irradiation time is significantly smaller than $30 \mathrm{~s}$.

The beam monitor output in 2-s intervals is shown below for the first four cycles of irradiation/counting from a typical irradiation[21] in Figure 3.4.2-1. The variation of the source strength during each cycle appears to be nearly constant both in terms of intensity and duration. It is assumed that this is true for all cycles. Because of the large slopes of the leading and trailing edges of the count rate distributions, it is assumed that the duration of the irradiation period is given by the full width at half maximum (FWHM) of the irradiation period. By use of linear fits to the two edges, the actual irradiation time is estimated to be $\square=26.3 \pm 0.5 \mathrm{~s}$. The cycle time derived from the leading edges of the first two irradiation cycles is $\mathrm{T}=129.7 \pm 0.5 \mathrm{~s}$, identical to the programmed time within estimated error.

\section{Scintillation Monitor Counts for Four Cy cles}

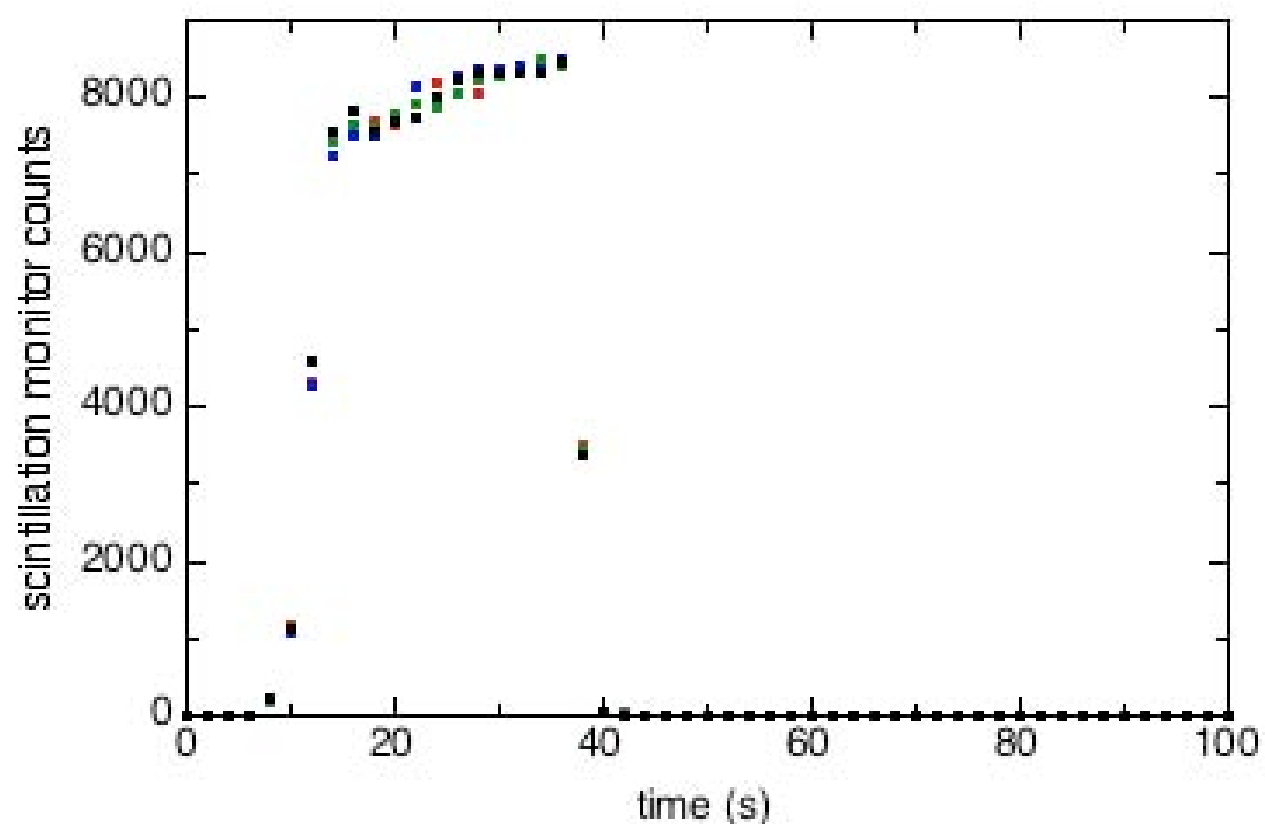

Figure 3.4.2-1 . Beam monitor counts for four cycles of $t_{\text {irr }}=30 \mathrm{~s}$ and $t_{\text {count }}=100 \mathrm{~s}$. The duration of a time bin is $2 \mathrm{~s}$ and the points are plotted at the time of the start of each bin. 


\subsubsection{Average Fission Yields of ${ }^{91.92} \mathrm{Sr}$ used to determine fission rate}

The recommended cumulative fission yields of ${ }^{91,92} \mathrm{Sr}$ reported by England and Rider[22] for thermal neutrons, for the prompt fission spectrum in a reactor and for $(\mathrm{d}, \mathrm{t})$ neutrons are shown in Table 3.4.3-1.

Table 3.4.3-1. Cumulative Fission Yields for ${ }^{91,92}$ Sr from England[22].

\begin{tabular}{|c|c|c|c|c|}
\hline & \multicolumn{2}{|c|}{ Cumulative Fission Yields (1 $\square$ error) (\%) } & \\
\hline Nuclide & Thermal & Fast & $(\mathrm{d}, \mathrm{t})$ & Adopted average \\
\hline${ }^{91} \mathrm{Sr}$ & $5.828(1.0)$ & $5.733(2.0)$ & $4.814(4.0)$ & $5.665(3.0)$ \\
\hline${ }^{92} \mathrm{Sr}$ & $5.938(1.4)$ & $5.843(2.0)$ & $5.063(11.0)$ & $5.793(3.0)$ \\
\hline
\end{tabular}

While the cumulative fission yields for thermal and fast neutrons are nearly identical, those for $14-\mathrm{MeV}$ neutrons are smaller by $15-20 \%$. Assuming the fission yields for fast reactor neutrons represent the average at energy of $2.0 \mathrm{MeV}$, the fission yields are nearly linearly dependent on neutron energy.

From the simulations of Hall[23] for a bare target located on axis of the neutron source and at a height of $111 \mathrm{~cm}$ above the laboratory floor, the fission rates predicted from thermal neutrons, 14.25-MeV neutrons and all other energies are in the ratios 1.00/1.09/0.387. The actual location of the target in most irradiations was at $140 \mathrm{~cm}$ above the laboratory floor and there is a small but significant variation in the relative intensity of thermal and source neutrons with distance from the collimator throat.

To estimate average cumulative fission yields for the two $\mathrm{Sr}$ isotopes, the simulated fluxes incident on a 7-cm (diam) surface surrounding the center of the target location has been used based on Descalle[20]. These fluxes were recorded in 7 energy groups spanning the range 0 14.25 MeV and were in very good agreement with the results of Hall[23]. Average cumulative yields were estimated with a linear variation of the fission yields with energy and use of the centroid energies of the groups. The results are shown in the last column of Table 3.4.3-1 along with estimated errors. The averages are within about $3 \%$ of the thermal yields notwithstanding the large contribution from fissions at the source energy. This is due primarily from the contribution of fissions from epithermal neutrons. The conclusion is that the uncertainty in the actual average fission yield of the two $\mathrm{Sr}$ isotopes cannot be in error by more than a few percent and the estimated errors shown in Table 3.4.3-1 should be conservative.

\subsubsection{Full-energy Efficiency of the HPGe Detector}

The HPGe detector used for assay of the $\square$ rays from decay of ${ }^{91,92} \mathrm{Sr}$ was a 14 -year old ORTEC device of the closed end coaxial type. It was stipulated to have a relative efficiency of $80 \%$ as delivered. (The original certification of this calibration is not now available.) However the device has been subjected to annealing at least once and thus was recalibrated in September 2004 by personnel in the Chemical Biology and Nuclear Science Division of LLNL[24]. It was found to have a relative efficiency of only $68 \pm 1 \%$ referred to the standard method of calibration by the manufacturer. 
Because of the extended nature of the HEU target, its strong attenuation for $\square$ rays and its complex counting geometry, the efficiency function applicable to our experimental arrangement was determined by a combination of Monte Carlo simulations using the dimensions of the Ge crystal and absolute normalization by measurements of the low-energy $\square$ rays from internal decay of the ${ }^{235} \mathrm{U}$ in the target, and by measurement of a calibrated ${ }^{60} \mathrm{Co}$ source that was fixed to the top of the target can and an identical container in the absence of the target.

The geometry of the target and detector in the experimental arrangement used in the counting experiments are illustrated are shown in detail by Descalle[25]. The can containing the HEU target was held in constant geometry by a lucite holder affixed to the detector housing. In this arrangement, the axis of the target intersected the axis of the Ge crystal. For most experiments, a $3.2 \mathrm{~mm}$ thick sheet of lead was inserted between the target and detector to reduce the interaction rate of the intense low-energy $\square$ rays from the decay of ${ }^{235} \mathrm{U}$. For counting of the first irradiation of the irradiated bare HEU target, the lead sheet was replaced by tantalum foils with a total thickness of $1.5 \mathrm{~mm}$.

\section{Decay counting Experiment}

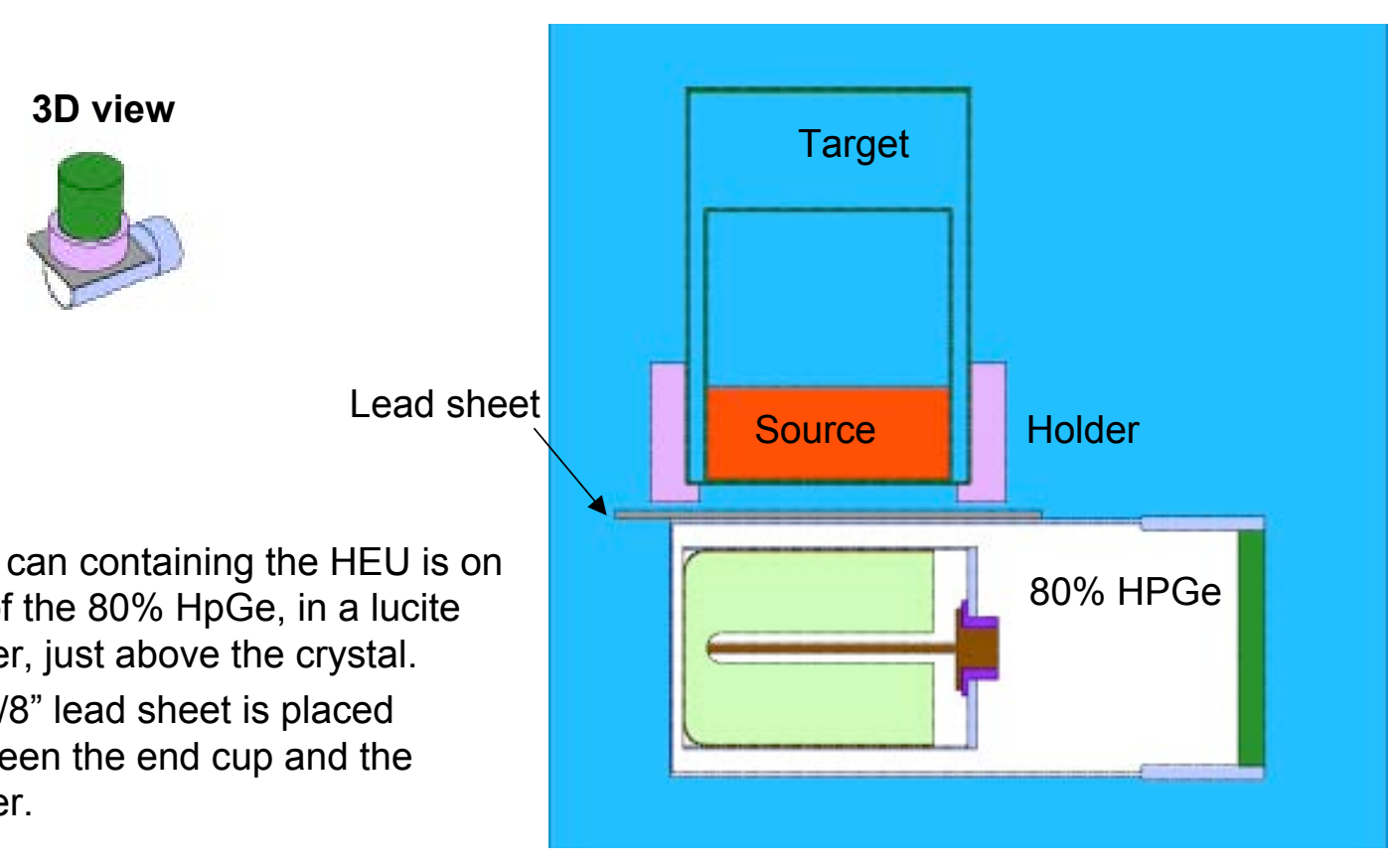

- The can containing the HEU is on top of the $80 \% \mathrm{HpGe}$, in a lucite holder, just above the crystal.

- A 1/8" lead sheet is placed between the end cup and the holder.

Figure 3.4.4-1. Geometry of modeling of the HPGe used for assay of the irradiated HEU target. 


\subsubsection{Monte Carlo Simulation of the Efficiency Function for the HPGe Detector and Normalization with Experimental Measurements}

The spectrum of energy depositions from monoenergetic photons arising uniformly from within the target was simulated with a detailed Monte Carlo model that included all of the features shown in Figure 3.4.4-1. The exact dimensions of the Ge crystal and its housing, as provided by the manufacturer, was used. However, the dead layers associated with the $\mathrm{p}$ - and $\mathrm{n}$-junctions were neglected. The simulated full-energy efficiency was taken as the fraction of events recorded in a $2-\mathrm{keV}$ wide region ending at the source energy and the results from these calculations due to Descalle[26] are shown in Figure 3.4.5-1. Included in the figure are the absolute efficiencies from the low-energy $\square$ rays emitted in the decay of the ${ }^{235} \mathrm{U}$ contained in the target when mounted in its normal position and the relative efficiencies at 583, 861 and 2614 $\mathrm{keV}$ from the decay of ${ }^{208} \mathrm{Tl}$, also contained in the target [27, 28]. The absolute efficiency data were normalized to the actual ${ }^{235} \mathrm{U}$ content of the target. The experimental and simulated efficiencies are in remarkable agreement at low energies, the average ratio of experiment to simulation being 0.95. However, the relative intensities of the 583-, 861- and 2614-keV lines from ${ }^{208} \mathrm{Tl}$ suggest that the slope of the simulated efficiency curve at higher-energies is not correct. This is shown in greater detail in Figure 3.4.5-2.

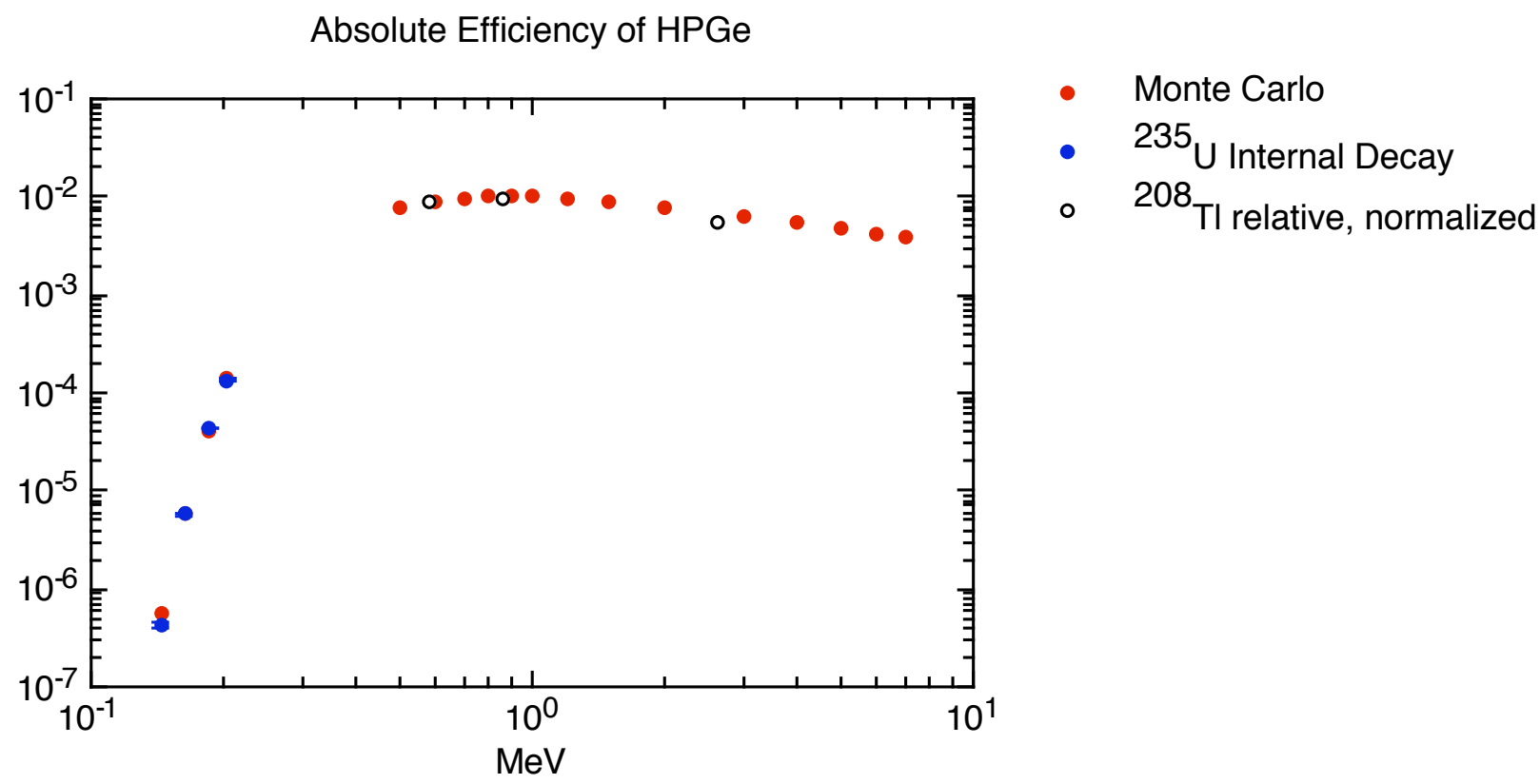

Figure 3.4.5-1. Simulated (red), experimental absolute efficiencies (blue) from ${ }^{235} U$ contained in the HEU target and relative efficiencies of the 583-, 861- and 2614-keV $\square$ rays from internal decay of ${ }^{208} \mathrm{Tl}$ normalized at the energy of $583 \mathrm{keV}$. 
Absolute Efficiency of HPGe

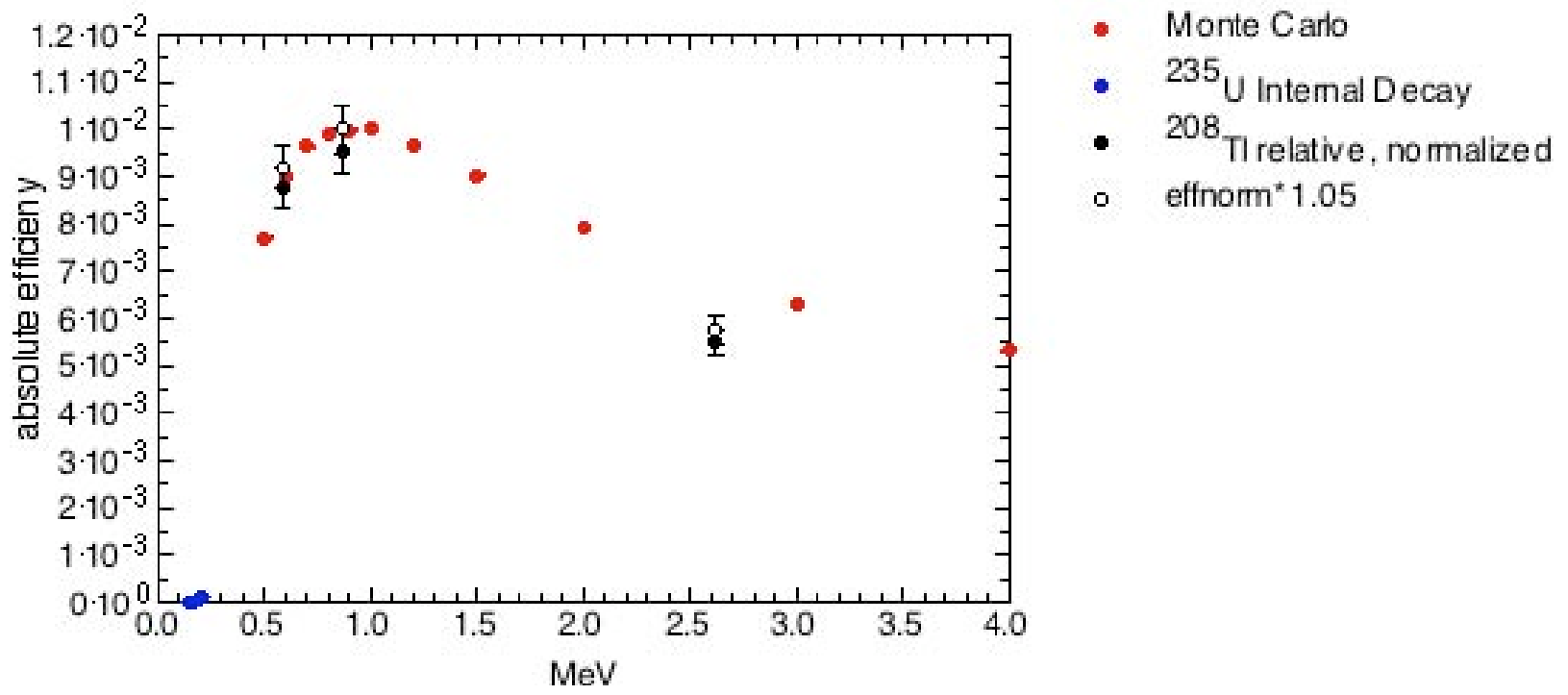

Figure 3.4.5-2. Efficiency of the HPGe on a linear scale. The relative efficiencies from the $\square$ rays emitted in the decay of ${ }^{208} \mathrm{Tl}$, normalized at $583 \mathrm{keV}$ and with the normalization increased by 5\%, are shown as the closed and open circles, respectively. The estimated errors in most measurements and the simulations are smaller than the data points. The relative efficiencies have been assigned reasonable errors of $\pm 2 \%$.

Clearly, the experimental efficiencies are smaller than those from the simulations at energies above about $0.6 \mathrm{MeV}$. Also as much as a $5 \%$ increase in the efficiency obtained from normalization at $583 \mathrm{keV}$ appears to produce an acceptable fit at both 583 and $861 \mathrm{keV}$. Taken together, the experimental efficiency at $2614 \mathrm{keV}$ must be smaller than that simulated by 1.32 1.39 .

In order to confirm the relation between the simulated efficiencies and reality, experiments were performed with a point source of ${ }^{60} \mathrm{Co}$ (NIST traceable) located at the center of the target can, with and without the presence of the HEU and with the lead shield in place[29]. These were then compared to simulations made under the same conditions[30]. The results are shown in Table 3.4.5-1.

Table 3.4.5-1. Comparison of simulated efficiencies for ${ }^{60} \mathrm{Co}$ पrays with experimental measurements for a source at the center of the top of the target container.

\begin{tabular}{|c|c|c|c|c|}
\hline Source & $\mathrm{E}_{\square}(\mathrm{keV})$ & $\mathrm{eff}_{\text {no target }}$ & eff $_{\text {target }}$ & $\mathrm{eff}_{\text {no targel }} /$ eff \\
\hline sarget \\
\hline & 1173 & $2.119 \pm 0.015(-3)$ & $1.150 \pm 0.010(-3)$ & $1.843 \pm 0.021$ \\
\hline & & & & \\
\hline Experiment & 1173 & $1.787 \pm 0.040(-3)$ & $8.865 \pm 0.196(-4)$ & $2.016 \pm 0.063$ \\
\hline & 1332 & $1.668 \pm 0.037(-3)$ & $8.665 \pm 0.191(-4)$ & $1.925 \pm 0.060$ \\
\hline
\end{tabular}

The ratios of the simulated to experimental efficiencies in the absence of the target are $1.186 \pm$ 0.028 and $1.206 \pm 0.028$ for the full energy peaks at 1173 and $1332 \mathrm{keV}$, respectively. They are 
to be compared with an on-axis efficiency that is smaller than the stipulated original efficiency at $1332 \mathrm{keV}$ by a factor of about 1.31 . Taken together, these results and those shown in Figure 3.4.5-1 tend to suggest that

- Charge collection near the upper surface of the detector cannot be significantly impaired relative to that which was present when the detector was first purchased. By inference, charge collection near the surface of the coaxial portion of the detector also cannot be significantly impaired.

- The main loss in efficiency is most likely due to charge collection problems in the lowfield regions near the closed end of the crystal and, possibly, from an increased dead layer thickness along the cylindrical central hole due to lithium diffusion during annealing.

When the model calculations were repeated with a $1 \mathrm{~mm}$-thick lithium dead layer and neglect of any events where the first interaction took place in the front portion of the detector that includes the non-axially symmetric fields, the ratios of the simulated to experimental efficiencies in the absence of the target were $0.974 \pm 0.023$ and $0.996 \pm 0.023$ [31]. These are in remarkable agreement and suggest that the inferences above are good. We will assume these simulated results in further analysis.

Turning to the efficiencies calculated in the presence of the HEU target, the ratios of the experimental and simulated efficiencies at 1173 and $1332 \mathrm{keV}$ are found to be $1.174 \pm 0.028$ and $1.187 \pm 0.029$. These ratios pertain to experimental measurements in the presence of only the outer container of the two-container experimental arrangement in the presence of the HEU target. Assuming that the inner container was composed of a lid and floor that were both $1 \mathrm{~mm}$ in thickness, the mean values of the ratios given above would be reduced to 1.080 and 1.091 , respectively, if the container was composed of pure iron, or to ratios of 1.038 and 1.049 if the container was composed of tin. We can conclude that, within errors of about $\pm 5 \%$, the simulations and experiments are in agreement. In summary, we can take all of the foregoing to define the absolute intensity of the HPGe under the normal counting conditions of the experiment.

The photon energies used to define the number of atoms of ${ }^{91} \mathrm{Sr}$ and ${ }^{92} \mathrm{Sr}$ produced in each target irradiation are $1024.3 \mathrm{keV}(33.4 \pm 2.3 \%)$ and $1383.93 \mathrm{keV}(90 \pm 3 \%)$, respectively. We then can approximate the efficiencies of the HPGe for counting of these $\square$ rays when emitted from the labeled target as $(9.60 \pm 0.48) \times 10^{-3}$ and $(8.65 \pm 0.43) \times 10^{-3}$, respectively.

\subsubsection{Correction for Attenuation by Tantalum}

The first experiment conducted on the irradiation of bare HEU target used 0.060 in of tantalum as an absorber for low-energy radiations rather than the $3 \mathrm{~mm}$ of lead used in all subsequent measurements. In order to determine the effective difference between the attenuation due to these absorbers, a Monte Carlo simulation was performed[31]. The efficiencies are shown in Figure 3.4.6-1. 


\section{Simulated Efficiencies with $\mathrm{Pb}$ and Ta Absorbers}

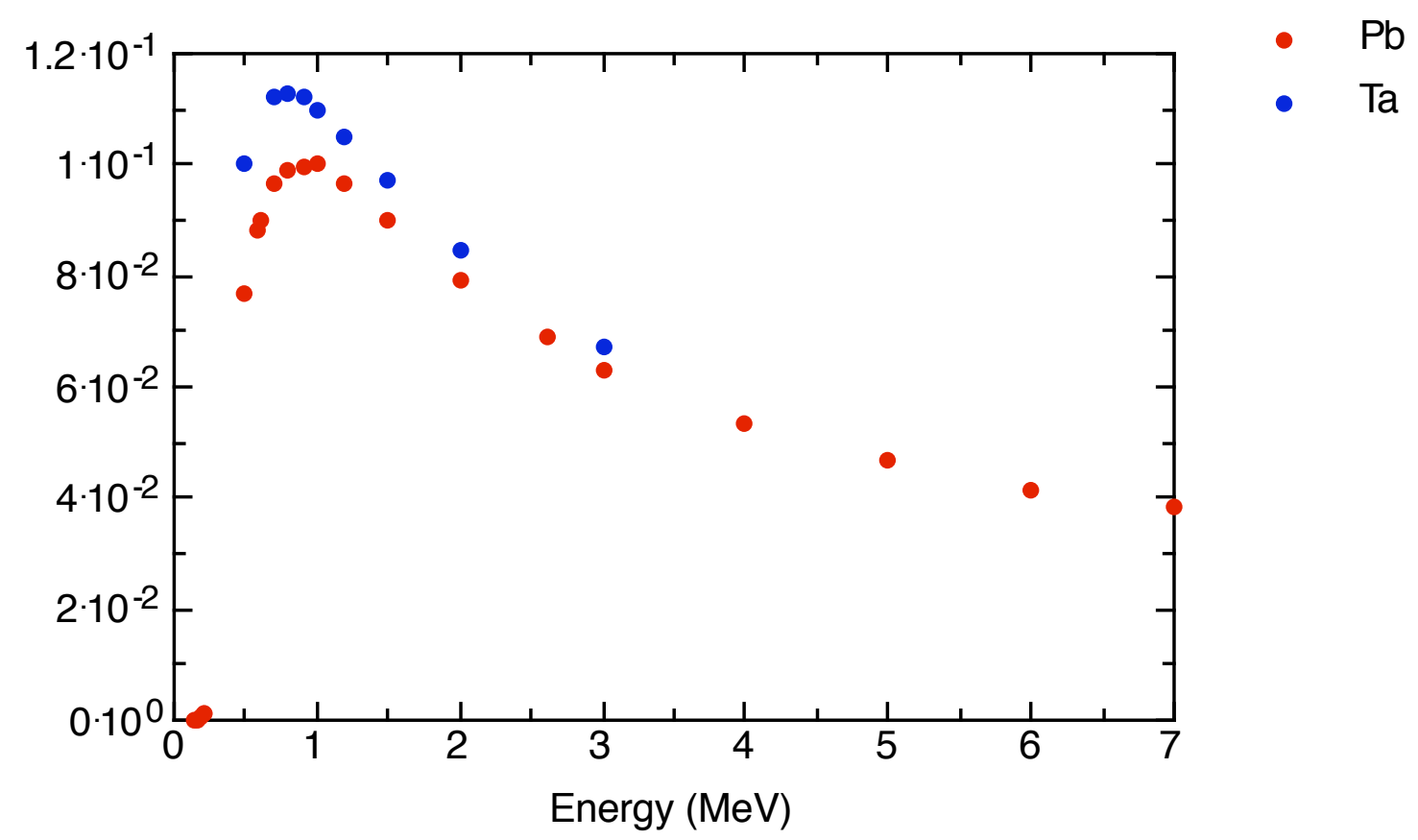

Figure 3.4.6-1. Comparison of the absolute efficiencies of the HPGe with $3.2 \mathrm{~mm}$ thick lead absorber and $1.5 \mathrm{~mm}$ thick Ta absorber.

The ratios of the efficiencies for the two absorbers are shown in Figure 3.4.6-2.

\section{Ratio of Efficiencies with $\mathrm{Pb}$ and $\mathrm{Ta}$ ab sorbers}

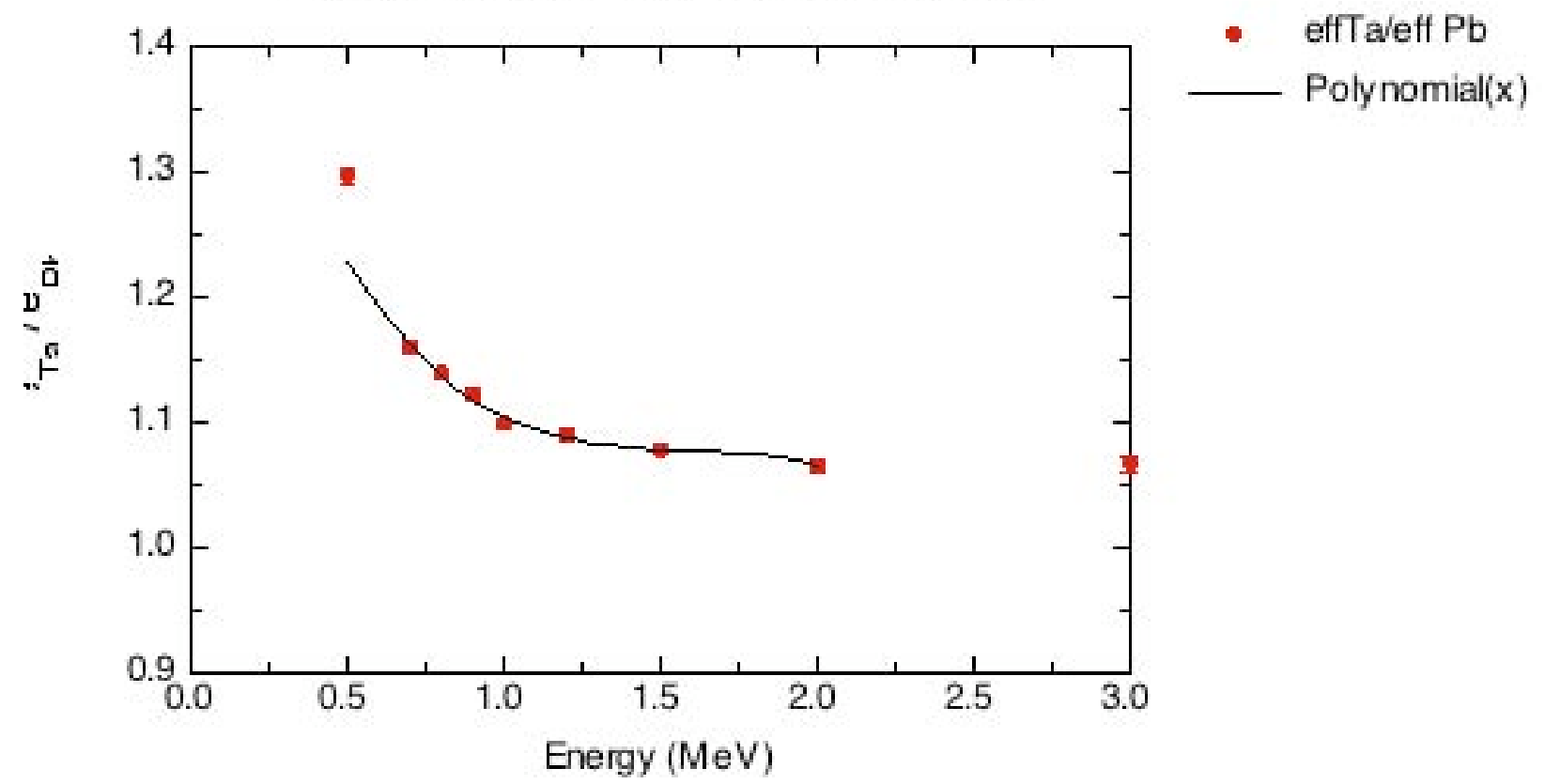

Figure 3.4.6-2. Ratio of efficiencies with tantalum and lead absorbers. The solid line represents a simple polynomial fit from which ratios can be interpolated in the energy range $0.7-1.5 \mathrm{MeV}$. 
It is clear that the efficiency ratios at 1024 and $1384 \mathrm{keV}$ are very nearly the same, and the efficiencies at these energies in the presence of the tantalum absorber are estimated to be (1.04 \pm $0.05) \times 10^{-1}$ and $(9.34 \pm 0.47) \times 10^{-2}$, respectively.

\subsection{Simulation of detector response characteristic}

We are now developing a "list mode" user-defined detector package available for COG which will be capable of simulating the acquisition and analysis of data from a series of repeated exposures (e.g. $N$ cycles of $30 \mathrm{sec}$ with beam on followed by $100 \mathrm{sec}$ with beam off (during which delayed gammas are recorded)). Coupled with our unique —-delayed †ray database[10]. This should allow us to model actual or proposed Active Cargo Interrogation experiments at LLNL with a hitherto unprecedented level of accuracy.

\subsection{Conclusions regarding code validation}

Experiments conducted with the $14 \mathrm{MeV}$ neutron source provided accurately measured fission rates by absolute counting of selected fission products after the irradiation. Details of those validation experiments are given later in Section 5. The measured values agreed with COG predictions to an accuracy $\sim 15 \%$. Rough measurements of the fray spectrum envelope agree with COG predictions and the measured and predicted intensities agree within a factor of $2 \mathrm{X}$. We consider that good agreement given the poor resolution of the spectral measurements and the extraordinary complexity of the 1000-2000 Gray lines in the region of interest.

Neutron fluxes from the generator have been measured and their spatial profile have been determined both along the collimator and transversely away from the beam axis. The measured profiles demonstrate that the COG predictions are highly accurate. These measurements have been made using diagnostic techniques developed for this purpose and the beam output has been continuously monitored during the measurements to insure that all variations were accounted for in the analysis.

A detailed model that describes neutron generation in a variety of D-D target concepts has been developed as an enhancement of the COG simulation tool. This model has not yet been tested but it provides the capability to predict detailed spectra and angular distributions for many candidate generator target concepts. 


\section{Detector evaluation}

We have evaluated organic liquid and plastic scintillator cells as detectors. The $\square$-delayed fission signature we seek to measure consists of a continuum of high-energy -rays in the range 3-6"MeV. The spectrum incident on detectors outside the cargo container has no pronounced lines, appearing as a superposition of falling exponentials in both time and energy. The $3 \mathrm{MeV}$ threshold eliminates the ambient background Grays, arising primarily from uranium, thorium and potassium, all of which lie below this threshold but does not eliminate background due to cosmic rays or activation products. This relatively low background and largely featureless signature is well suited to large solid angle, low resolution detectors such as liquid or plastic scintillator, which can be manufactured in square meter areas and 0.25-0.5 meter depths. We tested several models of this class of detector. We are using the $61 \mathrm{~cm}$ by $61 \mathrm{~cm}$ plastic cells, wrapped with a reflective multilayer sheet, with $25 \mathrm{~cm}$ thickness and single PMT readout. The main reasons for selection of these cells were:

- ease of handling and operation;

- lower environmental safety and health burden compared to the liquids,

- high intrinsic efficiency in the energy region of interest

- $\quad$ sufficient energy resolution with the multilayer wrap, and

- $\quad$ simplified analysis due to the single PMT readout.

Although liquid cells and the 2 PMT readout plastic cells had somewhat better energy resolution due to photostatistics or superior light collection (and the possibility of position sensitivity and position dependent energy correction in the case of the liquid cells), the single PMT plastic cells had sufficient energy resolution to allow application of the simple energy threshold method used in our analysis.

To learn about the properties of detectors, we tested one set of liquid cells and three types of $15 \mathrm{~cm}$ thick plastic cells. As described in more detail below, we ultimately chose a $25 \mathrm{~cm}$ thick plastic cell for the final set of detectors to be deployed, based on the increased efficiency for the 3-6 MeV gamma signal relative to the thinner $15 \mathrm{~cm}$ detectors.

\subsection{Description of the Detectors}

We evaluated four types of detector leading to the final choice for further studies of overall system performance. The configurations are shown schematically in Figure 4.1-1 and 4.1-2. 


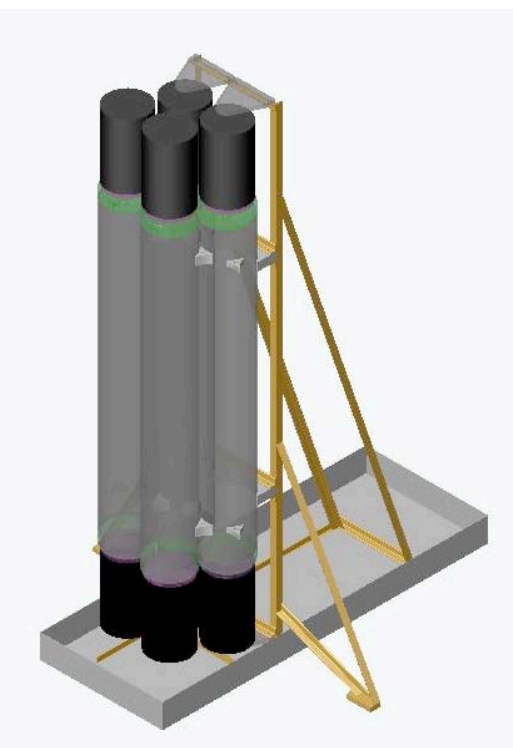

Fig. 4.1-1. The 4 liquid scintillator cells (Type I) shown as deployed. The active volume of the cells is $180 \mathrm{~cm}$ tall. The black end caps on top and bottom are mumetal shields surrounding $20 \mathrm{~cm}$ photomultiplier tubes.

They are:

- Type I - four $180 \mathrm{~cm}$ long, $20 \mathrm{~cm}$ diameter cylindrical position sensitive liquid scintillator cells, read out by $20 \mathrm{~cm}$ Electron Tubes 9354 photomultiplier tubes on either end of the cylinder (two tubes total per cell).

- Type II - A $61 \mathrm{cmt}$ by $61 \mathrm{~cm}$ square plastic scintillator cell with $15 \mathrm{~cm}$ depth, read on the back face by two $13 \mathrm{~cm}$ flat PMTs placed symmetrically along the diagonal.

- Type III - A $61 \mathrm{~cm}$ by $61 \mathrm{~cm}$ Square plastic scintillator cell with $15 \mathrm{~cm}$ depth, read out from the back by a single $13 \mathrm{~cm}$ flat PMTs placed in the center of the back face of the detector.

- Type IV - A $61 \mathrm{~cm}$ by $132 \mathrm{~cm}$ cell read out by two $13 \mathrm{~cm}$ PMTs placed on the top (61 $\mathrm{cm}$ by $15 \mathrm{~cm}$ ) side of the cell. 


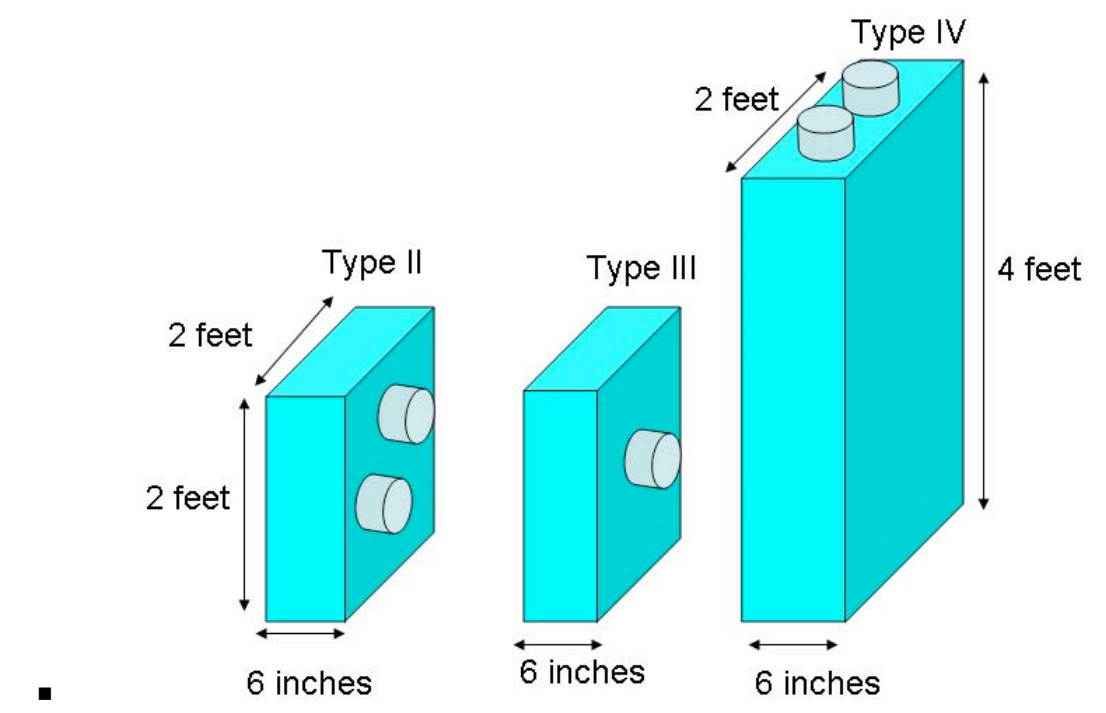

Fig. 4.1-2. Schematics of the Type II, II and IV cells, showing the location of the 5" PMTs and the cell dimensions.

The readout consists of a preamp-shaper combination, fed into a self-triggered FastComtec ADC and multiparameter analysis system. This system provides a timestamp with a $50 \mathrm{~ns}$ granularity for each event. Because of the modest detector resolution and to reduce the data word size, the ADCs are operated with 9 bit resolution.

We chose computer controlled multichannel high voltage controllers and shaping amplifiers. These have demonstrated good linearity and stability, and reliable and simple operation.

We gained experience with a shuttered first dynode for our PMTs and successfully used it throughout the first experimental campaign. The PMT first dynode is normally set at a bias of about $100 \mathrm{~V}$. Grounding this dynode during beam on periods allows us to quickly blind the PMTs to prevent anode saturation, without having to turn off the entire voltage divider chain in the PMT. This saves PMT lifetime compared to leaving the PMTS on through beam on periods (Which would quickly damage the dynode chain and anode), and compared to shutting them down completely for each beam cycle. The shuttering has worked well, and we have propagated the design to our new integrated PMT base, built to our spec by the vendor. This base contains a shutter, a built-in preamp and a test input to isolate and measure the gain from the preamp forward.

\subsection{Energy resolution}

We measured the effective resolution of the various detectors, here defined as the width of a ఈray peak in each detector as shown in Figure 4.2-1. 


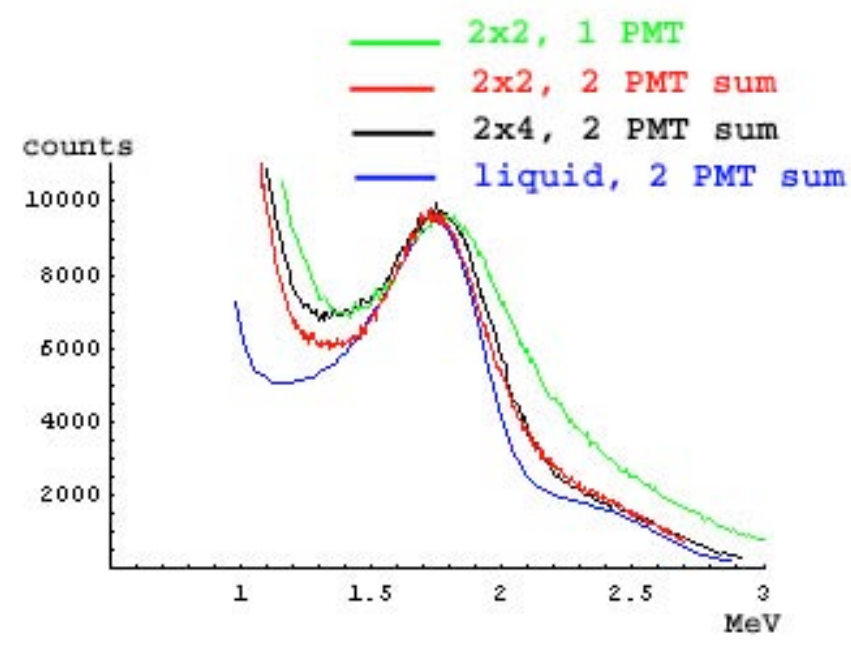

Figure 4.2-1. A comparison of the resolution for the 4 different cell types. The resolution is defined as the width of a Gaussian function fit to the upper lobe of the approximate $1.836 \mathrm{MeV}$ peak induced by ${ }^{88} Y$. An indication of the sum peak is visible in the liquid energy spectrum.

The resolution defined in this way depends on a variety of effects, including the detector geometry and the detection efficiency for Zrays in the region of interest, inhomogeneities in light collection, photostatistics, the PMT quantum efficiency, and the uniformity of the photocathode, and gain matching between PMTs in cases where two PMTs are summed (detector types I, II and IV). Table 4.2-1 below shows the FWHM resolution for the ${ }^{88} \mathrm{Y} 1.8$ $\mathrm{MeV}$ peak - after fitting for and subtracting an exponential background - for all cells.

Table 4.2-1 The effective energy resolution of four different cell types.

\begin{tabular}{|l|c|c|}
\hline Cell Type & $\begin{array}{l}\text { Mean energy (MeV) from } \\
\text { Gaussian fit to background } \\
\text { subtracted peak }\end{array}$ & $\begin{array}{l}\text { FWHM (= 2.35 } \square \text { with } \square \text { from } \\
\text { Gaussian fit to background } \\
\text { subtracted e peak) }\end{array}$ \\
\hline I- Liquid 2 PMT summed & 1.78 & $33 \%$ \\
\hline $\begin{array}{l}\text { II- 61x61 cm, 2 PMT } \\
\text { summed }\end{array}$ & 1.87 & $34 \%$ \\
\hline III-61x61 cm, 1 PMT & 1.82 & $50 \%$ \\
\hline $\begin{array}{l}\text { IV- 61x122 cm, 2 PMT } \\
\text { summed }\end{array}$ & 1.78 & $42 \%$ \\
\hline
\end{tabular}

Even without a correction of the energy scale for position, the liquid has a resolution similar to or slightly better than the plastic cell. The liquid shows a hint of the sum peak, which is not seen in the other cells. However, direct comparison with the liquid is not valid, because of detector geometry differences. The surest comparison is for the 1 PMT versus 2 PMT $61 \mathrm{~cm} \mathrm{X} 61 \mathrm{~cm}$ cells (types II and III). The 2 PMT resolution is $15 \%$ better in FWHM at the peak. As noted in 
the following section, we can recover the lost resolution in one PMT design (Type III) by using a highly reflective multilayer wrap.

Even with the poorest resolution detectors, we were able to discern a signal from HEU with approximately 10 cycles of $14 \mathrm{MeV}$ neutron irradiation, so that the resolution was not a decisive feature in selecting a detector. It is possible that a more sophisticated analysis could make use of additional resolution. For this purpose the low resolution single PMT detectors have been shown to suffice, particularly with the higher reflectivity multilayer wrap in place as described below.

We modified our plastic test cells by having the standard aluminum foil wrap replaced by a multilayer wrap (VM2000, manufactured by 3M). This had the effect of increasing the light yield by approximately a factor of two, due to the improved reflectivity of the multilayer. The effect is shown in Figure 4.2-1. This restores the resolution of the one PMT $61 \times 61 \mathrm{~cm}$ cell (type III) to approximately the value for type II (2 PMT readout), reduces the number of PMTs and simplifies the data taking and analysis steps.

In the liquid cells, we have shown that it is possible to correct the energy for position, and to define an approximate $20 \mathrm{~cm}$ right cylindrical voxel in which a gamma ray has interacted. It would be easy to reproduce this capability in a plastic cell of similar geometry. Figure 4.2-2 shows the correlation of the position of a collimated source with the quantity (ADC1$\mathrm{ADC} 2) /(\mathrm{ADC} 1+\mathrm{ADC} 2)$, which defines the ratio of difference and sum of the ADC values in opposite PMTs servicing a single liquid scintillator cell.

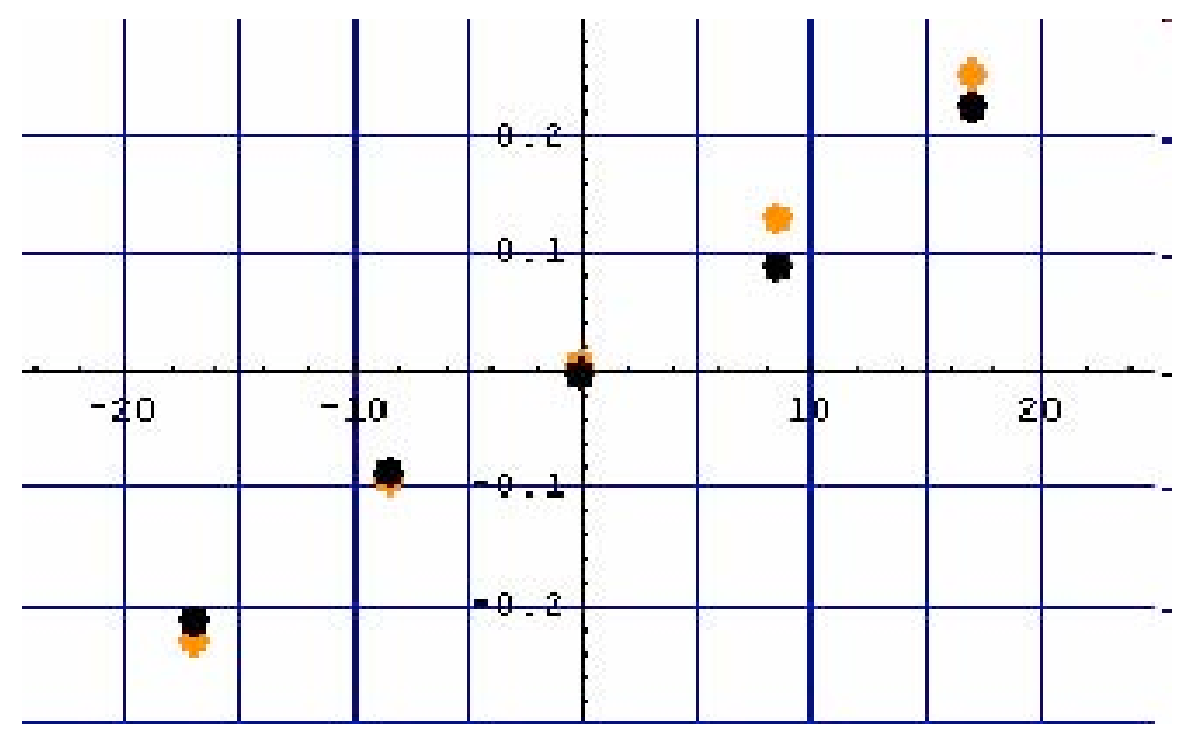

Figure 4.2-2. The $x$-axis is the position coordinate in inches of a collimated ${ }^{88} Y$ source along the cylindrical axis of a $180 \mathrm{~cm}$ liquid scintillator cell. The y axis is the ratiometric quantity, $(A D C 1-A D C 2) /(A D C 1+A D C 2)$. The two different sets of data points represent two different energies at which the ratio is calculated, showing that the relation between position and the ratio of anode charges (ADC values) is approximately independent of energy. 
The reconstructed position can be used to reduce the position dependence of the reconstructed energy in the detector. Without correction, the reconstructed energy for a given gamma peak varies by about ten percent across the cell. This dependence on position can be reduced to the 1$2 \%$ level by correcting the energy using a fit derived from the plot in Figure 4.2-2.

\subsection{Detection efficiency}

A central consideration is the intrinsic detection efficiency for 3-6 MeV Grays, here defined as the fraction of -rays of a given energy that deposit visible energy in the detector above a particular threshold. We calculated this efficiency by assuming that Compton interactions dominate the cross-section for the photons impinging on our low-Z hydrogenous detectors. This hypothesis is borne out by detailed Monte Carlo Gray transport simulations using the package MCNP, which demonstrate that nearly all interactions in a plastic detector of even $25 \mathrm{~cm}$ thickness are single or multiple Compton interactions, and not photoelectric or pair-production. It is then simple to estimate the efficiency by analytical means. For the plastic detectors, we chose a thickness $(25 \mathrm{~cm}$ ) that guaranteed $50 \%$ efficiency for a Compton scatter (of any energy) from $6 \mathrm{MeV}$ gammas incident on the detector. For each of the energies 3, 4, and $6 \mathrm{MeV}$, we then scaled the efficiency for that energy (at the $25 \mathrm{~cm}$ thickness), with the ratio

$$
\frac{\sigma_{\text {Compton }}\left(\theta_{\min }<\theta<180\right)}{\sigma_{\text {Compton }}(0 \rightarrow 180)}
$$

The numerator is the integral over solid angle of the Klein-Nishina differential scattering crosssection for scattering angles corresponding to a Compton electron deposition at or above the desired energy threshold, and the denominator is the same integral over all angles. The product of this ratio and the efficiency for a scatter of any energy with a fixed detector thickness approximates the efficiency for a scatter above a chosen energy.

In summary, for a $25 \mathrm{~cm}$ depth of plastic scintillator with unit density:

- At least $35 \%$ of $6 \mathrm{MeV}$ photons will deposit $>3 \mathrm{MeV}$ in the detector.

- At least $26 \%$ of $4 \mathrm{MeV}$ photons will deposit $>3 \mathrm{MeV}$.

- At least $30 \%$ of $3 \mathrm{MeV}$ photons will deposit $>2 \mathrm{MeV}$.

This assumes a normal flux of gammas. Since many gammas have longer straight through paths than the normal, the estimate should be conservative except near the edges of the detector.

With an average chord length of $13 \mathrm{~cm}$ for a planar flux impinging on our $20 \mathrm{~cm}$ diameter cylindrical cells, the efficiency (per unit of length along the cylinder axis, assuming a planar flux of gamma-rays) for two rows of close-packed, vertically oriented liquid scintillator array is about the same as for the $25 \mathrm{~cm}$ thick plastic detector. There may be additional advantages from the depth segmentation present in the liquid cell design, since multiple Compton scatters between detectors might be identified and used for crude directionality or background rejection. However, this complicates the analysis considerably and was not a reason to select the liquid 
cells. A future design"could incorporate the same potential advantage in a two-deep array of plastic detectors.

Since our analysis depends on the setting of an energy threshold for background rejection, it is important to study the stability of the energy calibration over times relevant for the experiment. We measured the stability of the liquid cells over short (tens of minutes) and long ( 24 hours) periods of time. Variations with temperature are apparent at the 5\% level comparing the hottest and coolest times of day. These can, in principle, be corrected by scaling with temperature. However, the best procedure is to control the detector temperature.

1.9 MeV peak: meanand error from gaussian fit

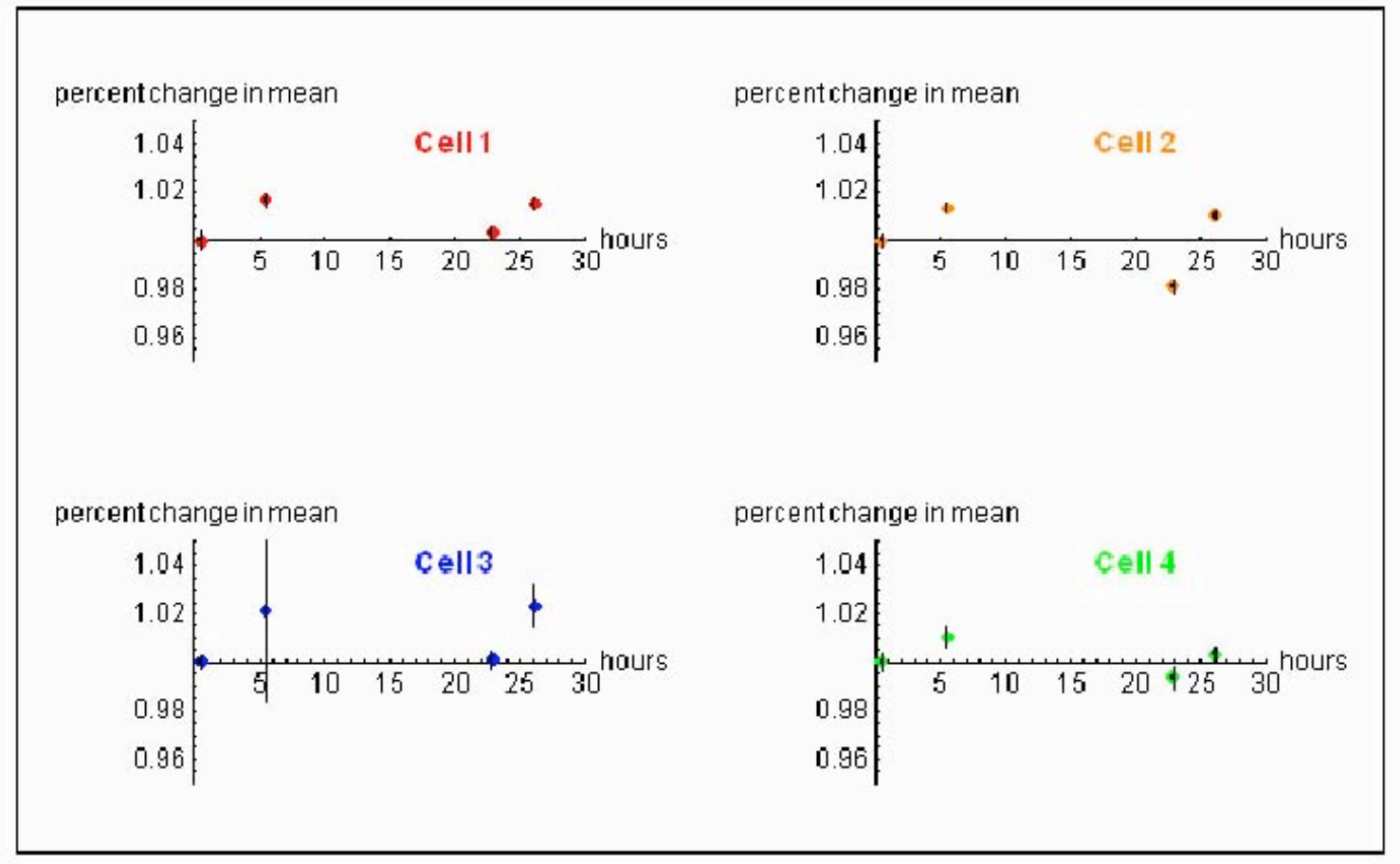

September 23 and 24 calibration data

Figure 4.3-1 The percent change in the $1.8 \mathrm{MeV}$ peak value for the liquid cells over a 24 hour period. Diurnal excursions due to temperature, which can be as large as several percent are not shown.

Figure 4.3-1 shows the stability of the calibration over a 24-hour period. Even without gain correction, the liquid cells are stable at the $1-2 \%$ level. This can be improved by a gain correction, provided calibration data are taken at suitably spaced intervals. The ultimate limitation is the error on fits of peaks in calibration data, which fits are used to extract the peak mean value. The fit errors in our calibrations are usually well below the few percent level.

We adopted a procedure of calibrating before and after each experimental run (duration 0.5-3 hours). Local temperature variations can worsen the calibration stability in both plastic and liquids, so temperature stabilization should be implemented in a final design. 
A lead sheet in front or around the detector can reduce the rate of low energy gammas. This in turn reduces detector event pile-up and dead time in the ADCs. Figure 4.3-2 shows the effect of a $3.2 \mathrm{~mm}$ thick lead blanket on 5 sides of a $30 \mathrm{~cm}$ square plastic detector. The overall reduction of rate above a low $(100 \mathrm{keV})$ threshold is about a factor of three.

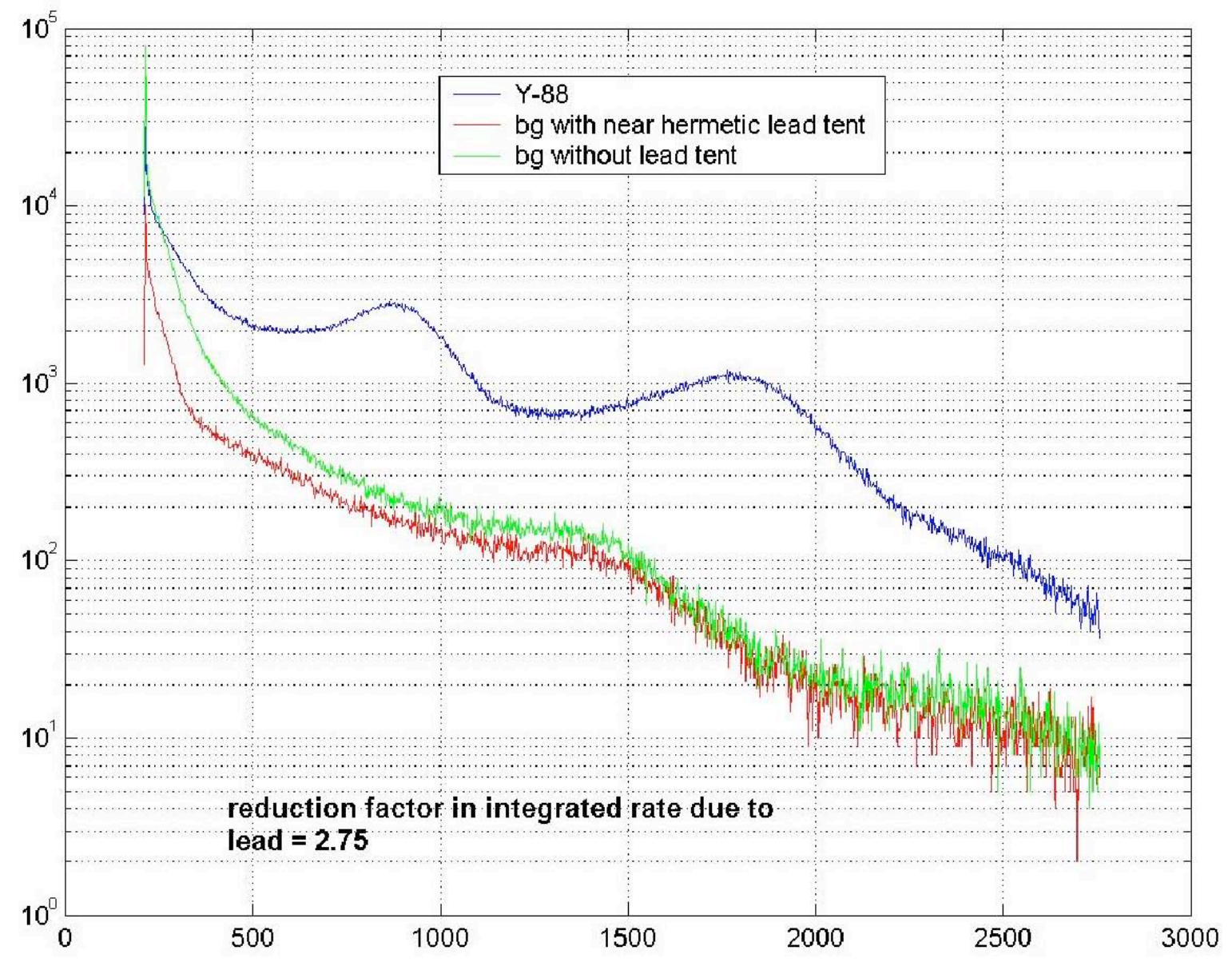

Figure 4.3-2. The effect of a near-hermetic lead blanket on the background spectrum in a 1 foot $x 1$ foot plastic scintillator detector. The integrated rate is reduced by a factor of 2.75 . 


\section{$5 \quad$ System feasibility demonstration and code validation}

During the course of developing components for a laboratory prototype it is essential to establish benchmarks to measure progress. For this purpose we periodically assembled candidate system components in the laboratory for a full-scale demonstration of overall system performance. These configurations included $\sim 400 \mathrm{~g} \mathrm{HEU}$ embedded in full-sized pallets of cargo surrogate materials. The pallets were loaded with $4 \times 8 \mathrm{ft}$ sheets of wood or similar layers of steel and stacked to a height $\mathrm{H} \sim 150 \mathrm{~cm}$. At least one large plastic scintillator and/or liquid scintillators was configured nearby to detect fission product radiation. These assemblies were irradiated from below for $\sim 30 \mathrm{sec}$ and the subsequent fission product radiation was recorded for another $100 \mathrm{sec}$. The result data acquired due to high-energy Gradiation was digested and displayed as a decay curve for comparison to simulations.

\subsection{Irradiation of Bare HEU Target at $14 \mathrm{MeV}$}

Detectors evaluated above were deployed near the $14 \mathrm{MeV}$ neutron source as the bare HEU target was irradiated at a distance of $140 \mathrm{~cm}$ above the floor of the experimental room. The principal experimental parameters for the experiments are shown in Table 5.1-1. The experiment duration, the number of irradiation/counting cycles and the total counts in the beam monitor were archived in the experiment record files[29, 32]. The mean generator output rate was calculated with the known relation between generator output and monitor count rate and measured irradiation duration. Note that the generator output calculated for the experiment is a factor of about 1.5 larger than the maximum output estimated from the calibration experiments.

The numbers of atoms of ${ }^{91,92} \mathrm{Sr}$ present at the end of the entire irradiation periods were determined by counting of the target on the HPGe. They depend only on the efficiency of the HPGe, the length of the count and the timing of the measurements with respect to the end of the entire irradiation period, and the small uncertainties on the decay properties of the isotopes[33]. The numbers of atoms predicted from the simulations were obtained with the simulated fission rate per source particle of $(6.75 \pm 0.07) \times 10^{-6}[34]$, the mean generator source strengths found from the beam monitor records, the known fission yields are shown in Table 5.1-1. The results are compared in Table 5.1-2.

Table 5.1-1. Principal parameters for the irradiations of the bare HEU target.

\begin{tabular}{|r|c|c|}
\hline Experiment No. & 1 & 2 \\
\hline Date & $09 / 17 / 04$ & $01 / 18 / 05$ \\
\hline Duration of experiment $(\mathrm{s})$ & 12997.14 & 6630.5 \\
\hline No. of cycles & 100 & 51 \\
\hline Beam monitor counts & $5.097 \times 10^{6}$ & $5.431 \times 10^{6}$ \\
\hline$<\mathrm{R}_{\mathrm{m}}>\left(\mathrm{s}^{-1}\right)$ & $1938 \pm 37$ & $4049 \pm 77$ \\
\hline$<\mathrm{S}_{14}>\left(\mathrm{s}^{-1}\right)$ & $(2.81 \pm 0.30) \times 10^{10}$ & $(5.87 \pm 0.62) \times 10^{10}$ \\
\hline
\end{tabular}


Table 5.1-2. Measured (exp) and predicted (sim) number of atoms of ${ }^{91}$.

\begin{tabular}{|c|c|c|c|c|c|c|}
\hline No. & $\begin{array}{c}\mathrm{n}_{\text {sim }}\left({ }^{91} \mathrm{Sr}\right) \\
\left(\times 10^{-7}\right)\end{array}$ & $\begin{array}{c}\mathrm{n}_{\text {sim }}\left({ }^{92} \mathrm{Sr}\right) \\
\left(\times 10^{-7}\right)\end{array}$ & $\begin{array}{c}\mathrm{n}_{\text {exp }}\left({ }^{91} \mathrm{Sr}\right) \\
\left(\mathrm{x} 10^{-7}\right)\end{array}$ & $\begin{array}{c}\mathrm{n}_{\text {exp }}\left({ }^{92} \mathrm{Sr}\right) \\
\left(\times 10^{-7}\right)\end{array}$ & $\begin{array}{c}\mathrm{n}_{\text {sim }} / \mathrm{n}_{\text {exp }} \\
{ }^{91} \mathrm{Sr}\end{array}$ & $\begin{array}{c}\mathrm{n}_{\text {sim }} / \mathrm{n}_{\text {exp }} \\
{ }^{29} \mathrm{Sr}\end{array}$ \\
\hline 1 & $2.48 \pm 0.27$ & $1.88 \pm 0.21$ & $2.00 \pm 0.11$ & $1.13 \pm 0.05$ & $1.24 \pm 0.14$ & $1.56 \pm 0.19$ \\
\hline 2 & $2.81 \pm 0.31$ & $2.44 \pm 0.27$ & $1.82 \pm 0.10$ & $1.59 \pm 0.09$ & $1.54 \pm 0.19$ & $1.53 \pm 0.19$ \\
\hline
\end{tabular}

The number of fission product atoms estimated from the simulations is generally larger than found in the counting experiments by about twice the estimated error. Considering the complexity of the experiments and the uncertainty in the exact geometry of the $\mathrm{U}_{3} \mathrm{O}_{8}$ target this order of agreement must be considered reasonable.

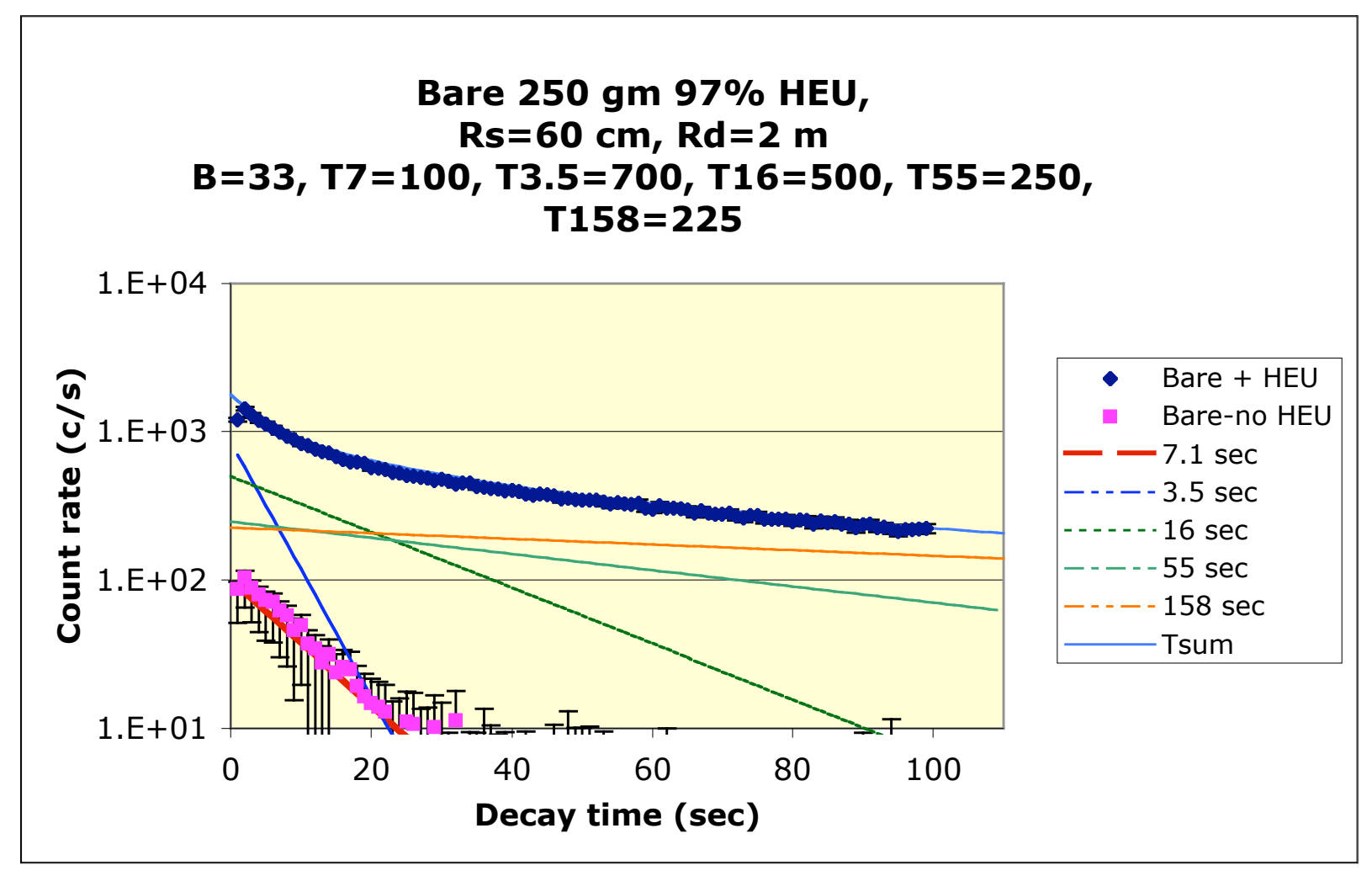

Figure 5.1-1 Decay curve showing count rate in the scintillation detector vs. time after irradiation. Colored lines indicate the estimated count rate due to the fission product groups characterized by the half lives 3.5, 16, 55, and $158 \mathrm{sec}$.

Data in the figure above shows detector count rates as a function of time after beam shutoff when the large plastic scintillation detector is operated with a discriminator that suppresses events that deposit less than $3 \mathrm{MeV}$ in the detector. Two data sets with estimated uncertainties are shown: a) "bare+HEU" which is the normal irradiation sequence, but constant background has been subtracted; and b) "bare-no HEU" which is a repeat of the same irradiation procedure but with the HEU removed from the beam. In both cases the irradiation was repeated $\sim 50$ times and the data summed for improved statistics and reduced error estimates. Nevertheless, the 
ordinate shows count rate vs. time for a single irradiation. The "bare-no HEU" exhibits the characteristic $7.1 \mathrm{sec}$ decay of ${ }^{16} \mathrm{~N}$ produced by the ${ }^{16} \mathrm{O}(\mathrm{n}, \mathrm{p}){ }^{16} \mathrm{~N}$ reaction but its count rate is $10 X$ less than is the case with HEU present. Several lines are shown on the plot to exhibit exponential decays with half-lives $3.5,16,55$, and $158 \mathrm{sec}$. These half-lives are representative of several groups of fission products known to produce significant Gradiation at energies above $3 \mathrm{MeV}$. The amplitudes of those exponential decays, together with a $7.1 \mathrm{sec}$ half-life decay have been adjusted and summed to produce the solid blue curve and their $y$-axis intercepts are given in the header. These exponential components provide an excellent fit to the data over the whole time range out to $t=100 \mathrm{sec}$. Overall, the upper data set shows a decay curve that can be accounted for by the decay of fission products and ${ }^{16} \mathrm{~N}$ alone. From this we conclude that, except for ${ }^{16} \mathrm{~N}$ the radiation signal is entirely due to the presence of the HEU. It has been shown before[5] that the dozen or so fission products that produce the majority of Gray intensity at energies above $3 \mathrm{MeV}$ have half-lives that can be effectively grouped into these four approximate time bins. The consequence is that the decay curve shown above is well represented by this grouping of these fission products.

Also shown in Figure 5.1-1 are the relative amplitudes of the four half-life groups. Data in the header shows the initial count rate due to each group at $\mathrm{t}=0$. The constant background was $\mathrm{C}=33$ $\mathrm{c} / \mathrm{s}$ and the initial count rate due to the fission products was $\mathrm{C}=700+500+250+225=1675 \mathrm{c} / \mathrm{s}$.

\subsection{Irradiation at $14 \mathrm{MeV}$ of HEU Target Embedded in a Steel-pipe Array}

The HEU target was irradiated while embedded in an array of steel pipes to simulate a cargo of medium atomic number. A photo of the cargo is shown in Figure 5.2-1 and a schematic illustrating its position relative to the neutron beam is shown in Figure 5.2-2.

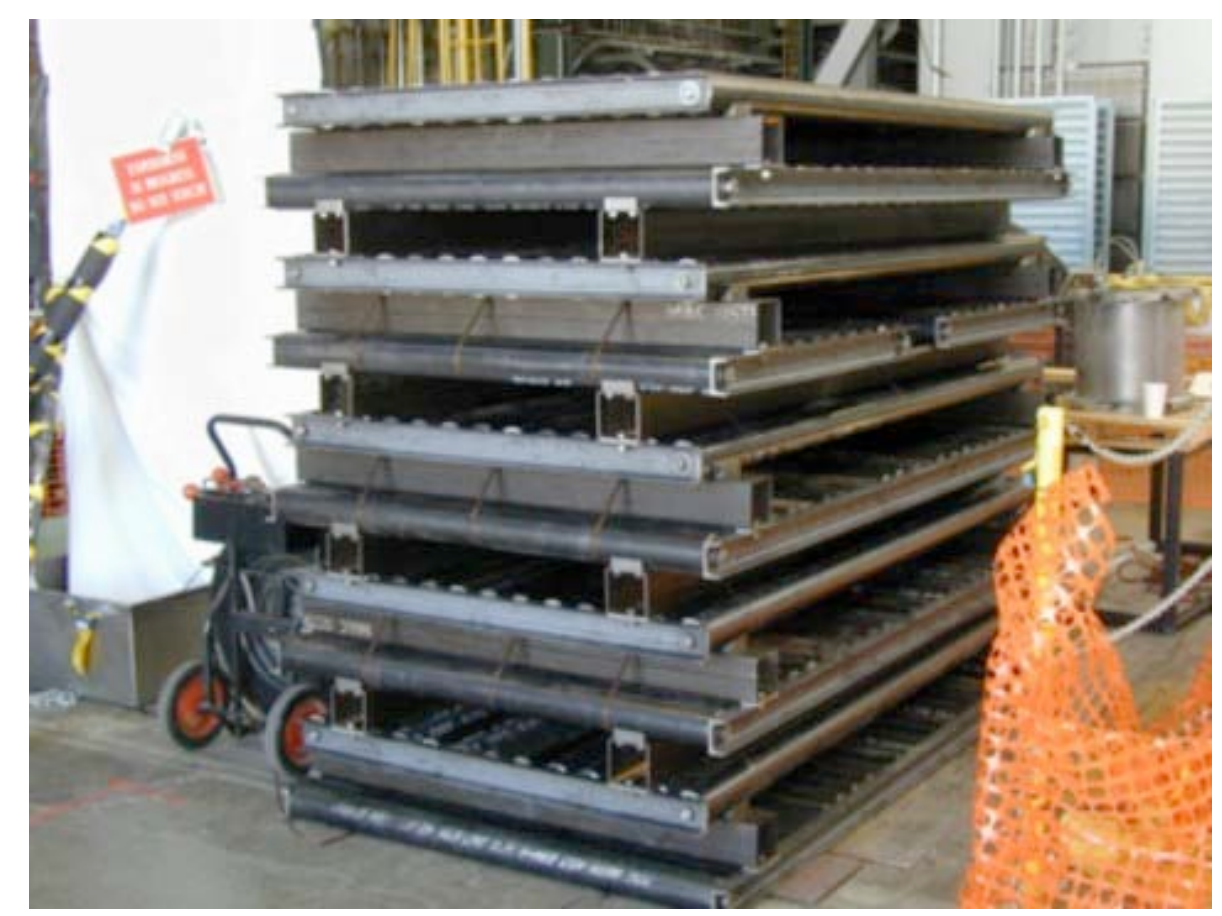

Figure 5.2-1 . . The steel pipe array representing a model of a high-Z cargo 


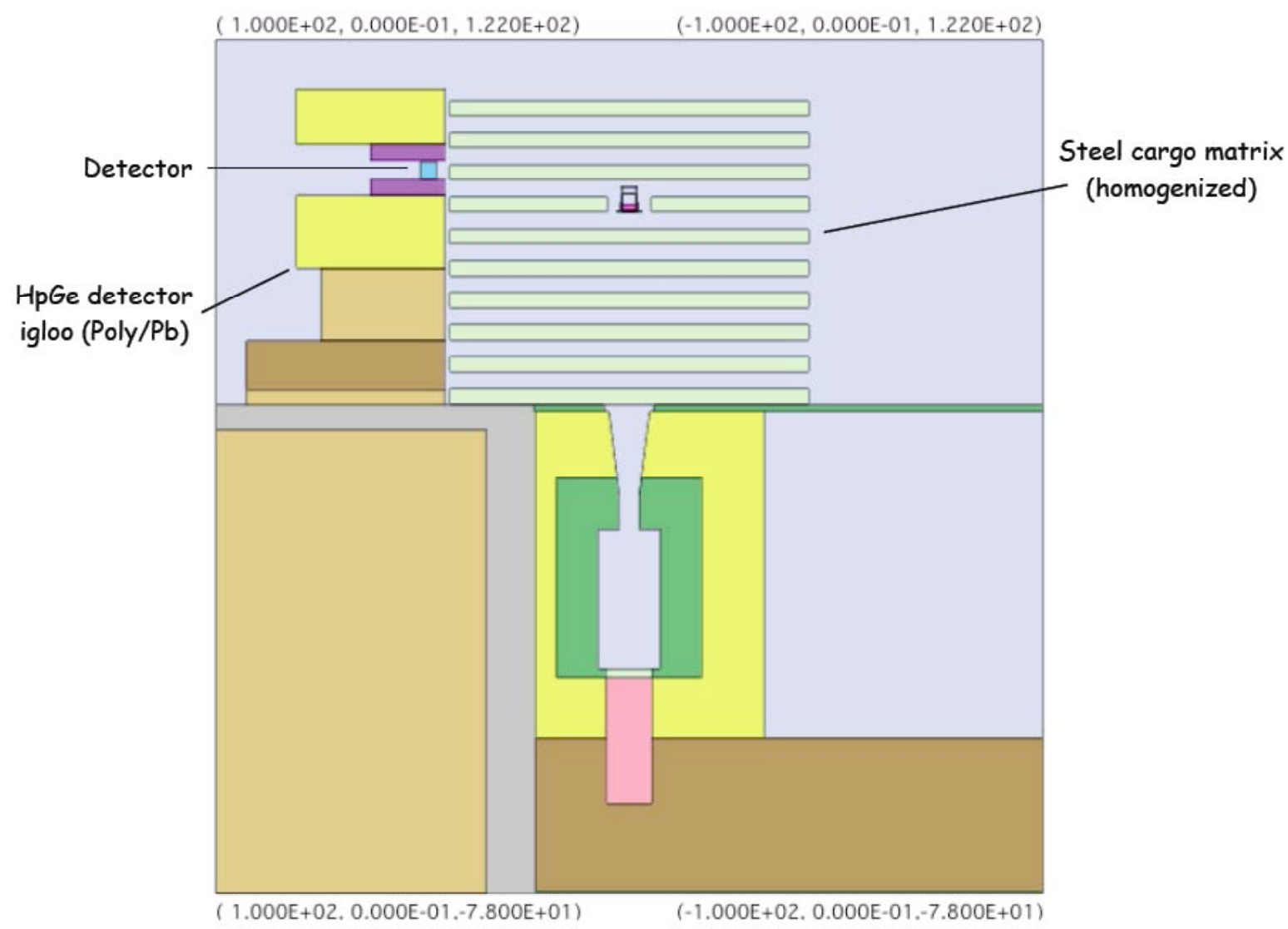

Figure 5.2-2. Schematic of the steel cargo consisting of a matrix of pipes arranged to give an average density of $0.6 \mathrm{~g} / \mathrm{cm}^{3}$.

The HEU target was located along the axis of the neutron beam at a height of $116 \mathrm{~cm}$ above the laboratory floor. The characteristics of the irradiation are given in Table 5.2-1 and the comparison between the measured (exp) and predicted ( $\operatorname{sim}$ ) number of atoms of ${ }^{91,92} \mathrm{Sr}$ is given in Table 5.2-2.

Table 5.2-1. Principal characteristics for the irradiation of HEU in the steel cargo.

\begin{tabular}{|r|c|}
\hline Experiment No. & 1 \\
\hline Date & $01 / 25 / 05$ \\
\hline Duration of experiment $(\mathrm{s})$ & 6630.0 \\
\hline No. of cycles & 51 \\
\hline Beam monitor counts & $5.930 \times 10^{6}$ \\
\hline$<\mathrm{R}_{\mathrm{m}}>\left(\mathrm{s}^{-1}\right)$ & $4421 \pm 84$ \\
\hline$<\mathrm{S}_{14}>\left(\mathrm{s}^{-1}\right)$ & $(6.41 \pm 0.67) \times 10^{10}$ \\
\hline
\end{tabular}


Table 5.2-2. Measured (exp) and predicted ( experiment.

\begin{tabular}{|c|c|c|c|c|c|c|}
\hline No. & $\begin{array}{c}\mathrm{n}_{\text {sim }}\left({ }^{91} \mathrm{Sr}\right) \\
\left(\times 10^{-6}\right)\end{array}$ & $\begin{array}{c}\mathrm{n}_{\text {sim }}\left({ }^{92} \mathrm{Sr}\right) \\
\left(\times 10^{-7}\right)\end{array}$ & $\begin{array}{c}\mathrm{n}_{\text {exp }}\left({ }^{91} \mathrm{Sr}\right) \\
\left(\mathrm{x} 10^{-6}\right)\end{array}$ & $\begin{array}{c}\mathrm{n}_{\text {exp }}\left({ }^{92} \mathrm{Sr}\right) \\
\left(\times 10^{-7}\right)\end{array}$ & $\begin{array}{c}\mathrm{n}_{\text {sim }} / \mathrm{n}_{\text {exp }} \\
{ }^{91} \mathrm{Sr}\end{array}$ & $\begin{array}{c}\mathrm{n}_{\text {sim }} / \mathrm{n}_{\text {exp }} \\
{ }^{2} \mathrm{Sr}\end{array}$ \\
\hline 1 & $9.93 \pm 1.10$ & $8.63 \pm 0.96$ & $6.67 \pm 0.36$ & $5.72 \pm 0.31$ & $1.49 \pm 0.19$ & $1.53 \pm 0.19$ \\
\hline
\end{tabular}

\subsection{Irradiation at $14 \mathrm{MeV}$ of HEU Target Embedded in Plywood}

The HEU target was irradiated while embedded in a stack of plywood sheets to simulate a typical hydrogenous cargo. A schematic is shown in Figure 5.3-1. The density of the plywood was measured to be $0.58 \mathrm{~g} \mathrm{~cm}^{-3}$ and the target was located at a height of $76.2 \mathrm{~cm}$ above the floor of the laboratory.

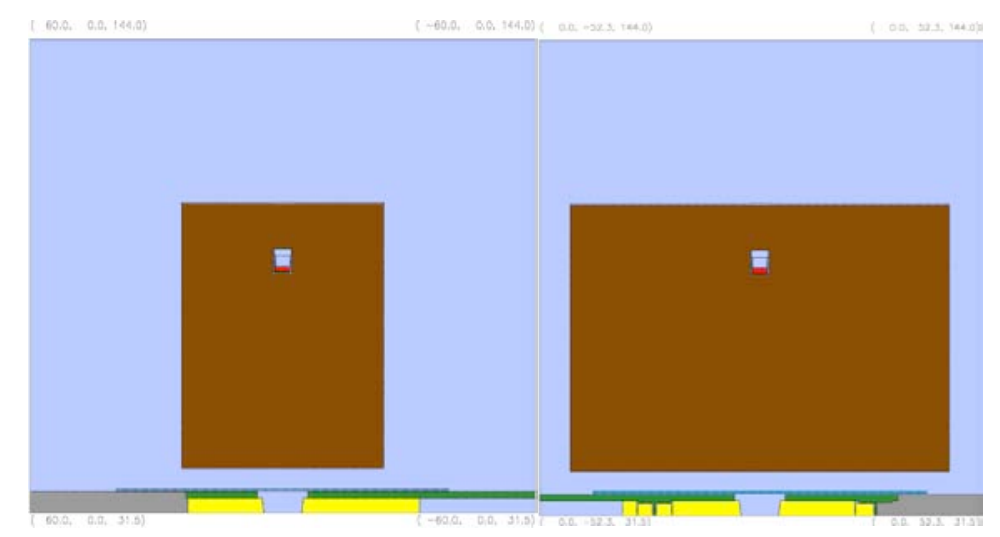

Figure 5.3-1. Schematic diagram of the plywood block chosen to represent hydrogenous cargo. The dimensions of the block are $122 \mathrm{~cm}^{\prime}$ (wide) $\times 244 \mathrm{~cm}$ (length) $\times 183 \mathrm{~cm}$ (height). 


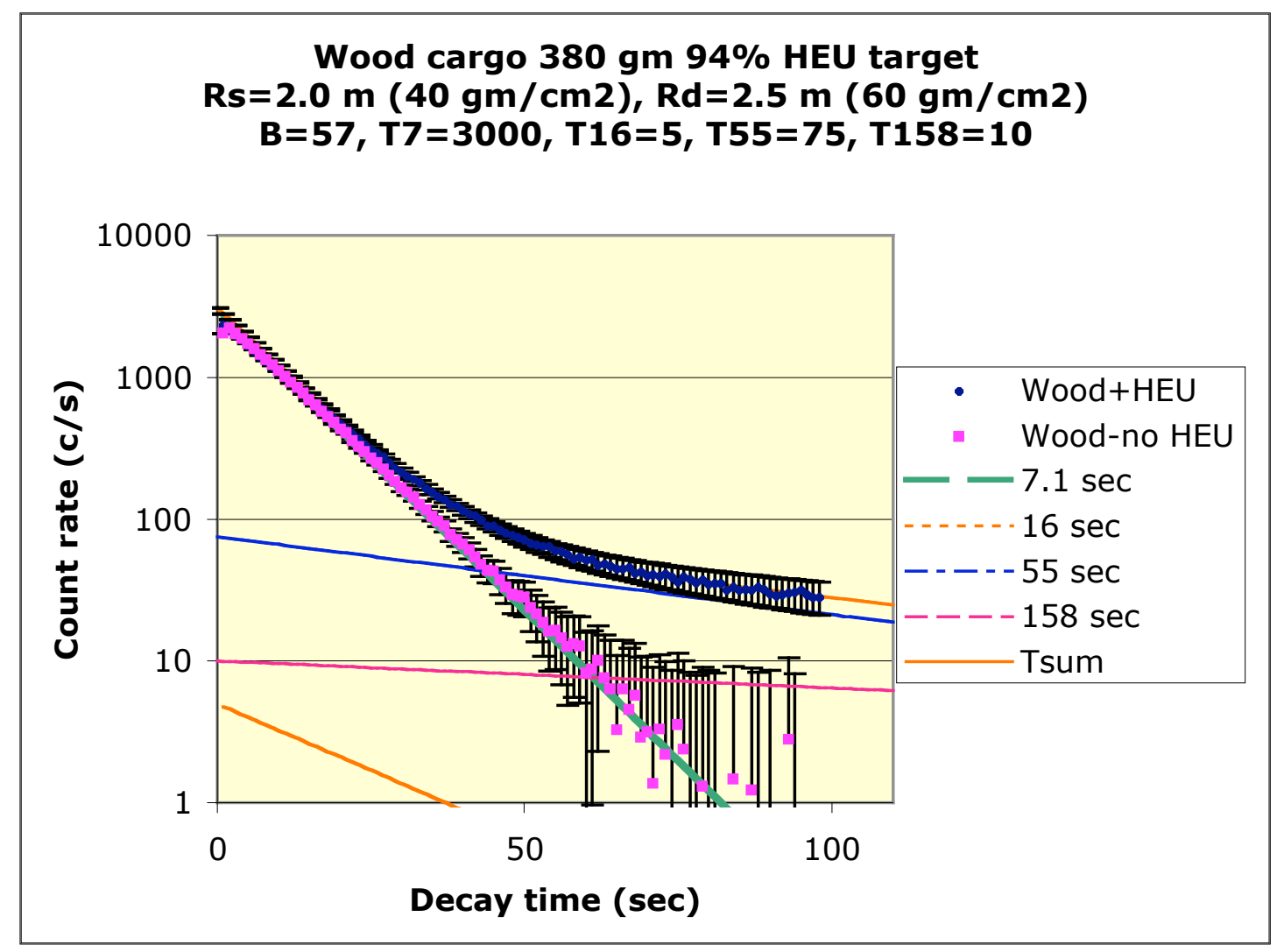

Figure 5.3-2. Decay curve showing count rate of Gray events depositing E $>3 \mathrm{MeV}$ in the scintillation detector.

The beam passed through $40 \mathrm{~g} / \mathrm{cm}^{2}$ of plywood to reach the HEU target, and the exiting $\square$ radiation passed through $60 \mathrm{~g} / \mathrm{cm}^{2}$ to reach the detector. As in Section 5.2 two data sets are shown, one with the normal irradiation (upper) and a repeat of the same irradiation but with HEU target removed (lower). Each set is shown with estimated uncertainties and the constant background has been subtracted. At early times the decay is dominated by oxygen activation, i.e. $7.1 \mathrm{~s}{ }^{16} \mathrm{~N}$ decay. However, at late times $(\mathrm{t}>50 \mathrm{sec})$ the fission product radiation is at least a decade more intense than that due to the ${ }^{16} \mathrm{~N}$. As before, the figure shows several exponential decays characteristic of those fission products that produce the majority of high-energy $\left(\mathrm{E}_{\square}>3\right.$ $\mathrm{MeV}$ ) radiation. The shortest lifetime group is masked entirely by the ${ }^{16} \mathrm{~N}$ background but the others are each critical to the final fit of the decay data. Removal of any among the remaining three fission product exponentials makes it impossible to provide a good fit to the decay data.

Parameters in the header to the figure show adjusted intercepts of the exponentials, i.e. their respective count rates at $\mathrm{t}=0$. The constant background was $57 \mathrm{c} / \mathrm{s}$. The fission product count rate at $\mathrm{t}=0$ is $\mathrm{C}=5+75+10=90 \mathrm{c} / \mathrm{s}$ with the wood cargo in place and with a neutron generator output nearly identical to that in the case of the bare HEU target described in Section 5.1. Our overall conclusion is that the $14 \mathrm{MeV}$ neutron generator producing $\sim 6 \times 10^{10} \mathrm{n} / \mathrm{s}$ and a single large scintillation detector provide a very good $\mathrm{S} / \mathrm{N}$ figure of merit at late counting times even in 
the presence of the oxygen activation. Hydrogenous cargo such as this one is the greatest challenge to neutron interrogation and data here clearly shows a robust signal through thicknesses of 40-60 $\mathrm{g} / \mathrm{cm}^{2}$ of wood cargo.

\subsection{Prediction of error rates}

The system requirements stated at the beginning addressed the need for $\sim 95 \%$ detection probability for the goal quantities of SNM in a variety of cargos. It also addressed the need for a very low rate of false positive detections. The goal here is $\mathrm{P}_{\mathrm{fp}} \leq 0.001$ false positive detections. The detection methodology employed here could be as simple as measuring the radiation intensity observed above a preset energy threshold as described in the introduction. Or it could be more complicated employing analysis of the radiation energy spectra, the temporal decay of the radiation intensity following irradiation, and even localization of a radiation hot spot as it moves along the detector array. All of these approaches will be evaluated in the next phase of this project. In the mean time a few simple analyses have been carried out to establish a rough perspective on the potential performance to be expected.

\subsubsection{The simplest analysis}

In the simplest case we would set up an array of Gray detectors and record only those events that deposit an energy $\mathrm{E}_{\square}>3 \mathrm{MeV}$, or some other suitably chosen threshold. A background count rate would be determined for that configuration and any container that produces a count rate significantly higher than background would generate a detection alarm. Then there are two questions:

- What is the detection probability for the defined configuration, i.e. what fraction of real SNM targets will escape detection?

- What is the false positive probability, i.e. what fraction of innocent cargos will generate a detection alarm?

Both questions are answered by an understanding of the distribution function of count rates for the configuration established. This is illustrated in the figure below. 


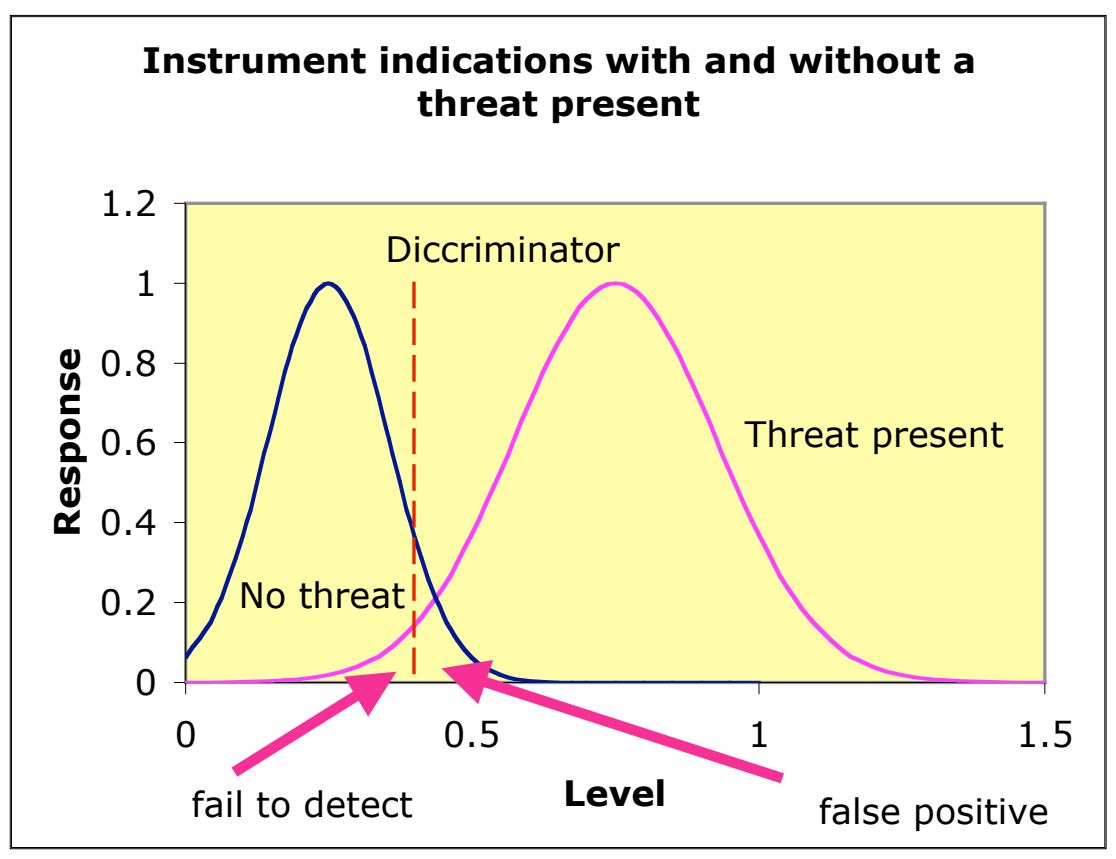

Figure 5.4.1-1 Normal distributions of detector count rates. The curve on the left shows normal variations in background count rate. On the right is shown the normal variations in count rate when SNM is present and generating a signal.

Radioactive decay is a Markov process meaning that the expected outcome of any event is independent of the history of previous events. The number of decays in any time interval is described by the binomial distribution. However, if the number of events is more than just a few then this distribution is well approximated by the Poisson distribution. And if the number of events is hundreds then the Gaussian distribution is a good approximation to the number of decays in any time interval. The curves in the figure above reflect the Gaussian approximation. On the left is shown a distribution of count rates due to background radiation detection when no SNM is present. This would correspond to the rate of background events that deposit energy in the detector exceeding the preset energy threshold. Then we would set a count rate threshold where any count rate exceeding that threshold would generate an SNM detection alarm. This is illustrated in the figure. Normal variations in the background count rate can produce rates above the preset threshold and will then generate false positive detections, i.e. generate an alarm when no SNM is present. The count rate distribution function is given below and probability of a false positive detection is the integral of the distribution above the preset count rate threshold.

$$
C_{B}\left(n,<C_{B}>, \square_{B}\right)=\frac{1}{\sqrt{2 \square \square_{B}}} e^{\square \frac{\left(n \square<C_{B}>\right)^{2}}{2 \square_{B}^{2}}}
$$


where $\square_{\square}^{2}$ is the variance in the background count rate, $\left\langle\mathrm{C}_{\mathrm{B}}>\right.$ is the mean count rate, and $\mathrm{n}$ is the number of counts in a predetermined observation interval. The Error Function for a preset threshold count rate $\mathrm{L}$ gives the probability of false positive.

$$
P_{f p}=\square_{L} C_{B}\left(n,<C_{B}>, \square_{B}\right) d n=\frac{1}{2}\left[1 \square \operatorname{erf}\left(x_{1}\right)\right]
$$

where

$$
x_{1}=\frac{L \square<C_{B}>}{\square_{B} \sqrt{2}}
$$

and

$$
\operatorname{erf}(x) \equiv \frac{2}{\sqrt{\square}} \square_{0}^{x} e^{\square t^{2}} d t
$$

Evaluation of the above equation shows that the requirement $\mathrm{P}_{\mathrm{fp}} \leq 0.001$ is met only when the following condition is satisfied:

$$
L \geq 3.3 \square_{B}+<C_{B}>
$$

Similarly, the setting of the count rate threshold determines the detection probability with signal present.

$$
P_{D}=\prod_{L} C_{S}\left(n,<C_{B}>+S, \square_{B}\right) d n=\frac{1}{2}\left[1+\operatorname{erf}\left(x_{2}\right)\right]
$$

where we've assumed $\square_{\mathrm{S}}^{2}<<\square_{\mathrm{B}}^{2}$, i.e. the signal detection rate variance is small in all cases requiring ROC analysis, and the signal strength is $\mathrm{S}$ so that the mean detection rate with $\mathrm{SNM}$ present is $\left\langle\mathrm{C}_{\mathrm{S}}\right\rangle=\mathrm{S}+\left\langle\mathrm{C}_{\mathrm{B}}\right\rangle$. The parameter $\mathrm{x}_{2}$ is given by:

$$
x_{2}=\frac{<C_{B}>+S \square L}{\square_{B} \sqrt{2}}
$$

Evaluation of the above equation shows that $\mathrm{P}_{d} \geq 95 \%$ only when the following condition is met.

$$
<C_{B}>+S \geq L+1.7 \square_{B}
$$

\subsubsection{The ROC curve and the 5xSIGMA rule}

The first conclusion we reach is that there is no simultaneous solution to (5.4.1-5) and (5.4.1-8) unless the following condition is met:

$$
S \geq 5 \square_{B}
$$


The detected SNM signal must exceed the mean background count rate by about five standard deviations in order to allow $95 \%$ detection probability and 0.001 false positive probability; and the count rate threshold must be set to a count rate 3.3 standard deviations above the mean background rate for the chosen observation interval.

The analysis in the previous section can be generalized and interpreted in the form of a set of curves where $\mathrm{L}$ is varied at constant $\mathrm{S}$. Sometimes a set of curves is generated for different detected signal levels. These curves are called ROC (Receiver Operating Characteristic) curves.

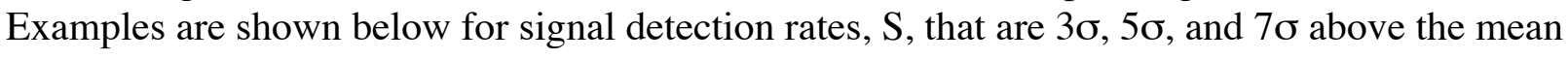
background.

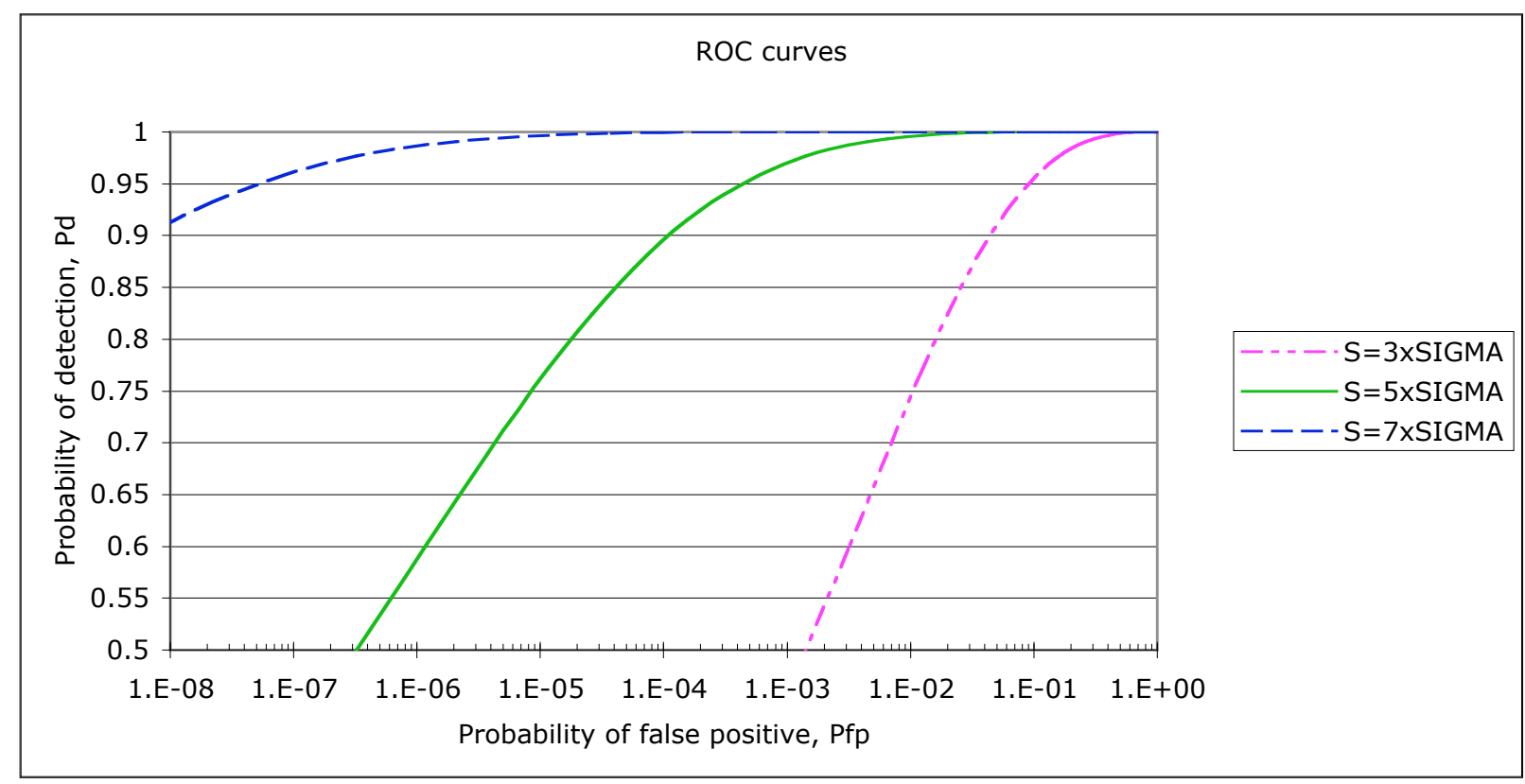

Figure 5.4.2-1 ROC curves for signal detection rates that are 3, 5, and 7 standard deviations above the mean background count rate.

Examination of the figures shows that changes in threshold L can tradeoff large changes (decades) in false positives and false negatives. Even more important, small changes in $\mathrm{S}$ can greatly change the accessible ranges of $\mathrm{P}_{\mathrm{d}}$ and $\mathrm{P}_{\mathrm{fp}}$. Note in the figure that as the signal detection

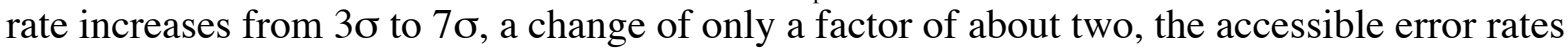
change by many decades. This establishes a breakpoint at $\mathrm{S} \sim 5 \square$ below which the signal detection rate is too low to allow both high detection probability and low false positives regardless of threshold $\mathrm{L}$, and above which high detection probability is attainable even at extraordinarily low false positive rates. In later analyses the predicted signal detection rate $S$ will be determined and compared to this standard, i.e. is $S \geq 5 \square$ ?

These analyses can include known distribution functions for the detector background count rates to determine the signal strength $S$ required to meet any proposed requirements. The validity of such analyses is determined by the extent to which the actual count rate distribution conforms to 
the Gaussian description utilized above. For these analyses to be predictive the critical part of the representation of counting statistics is at large distances from the mean. Thus, whether the Gaussian representation at several standard deviations above the mean is a good is the critical question to be answered empirically.

\subsubsection{What we do and don't know about the count rate distribution functions}

Background count rates vary because of:

- Random radioactive decay events

- Cosmic ray showers that are intermittent

- Movement of shielding in the environment where measurements are being made

- Movement of radioactive material including materials activated by the neutron interrogation.

All of these sources of background variation can contribute to false positive errors and not all of them are well understood. Measurements have been made in the cargo-scanning lab to assess the variations in background count rate while materials are not being moved. 


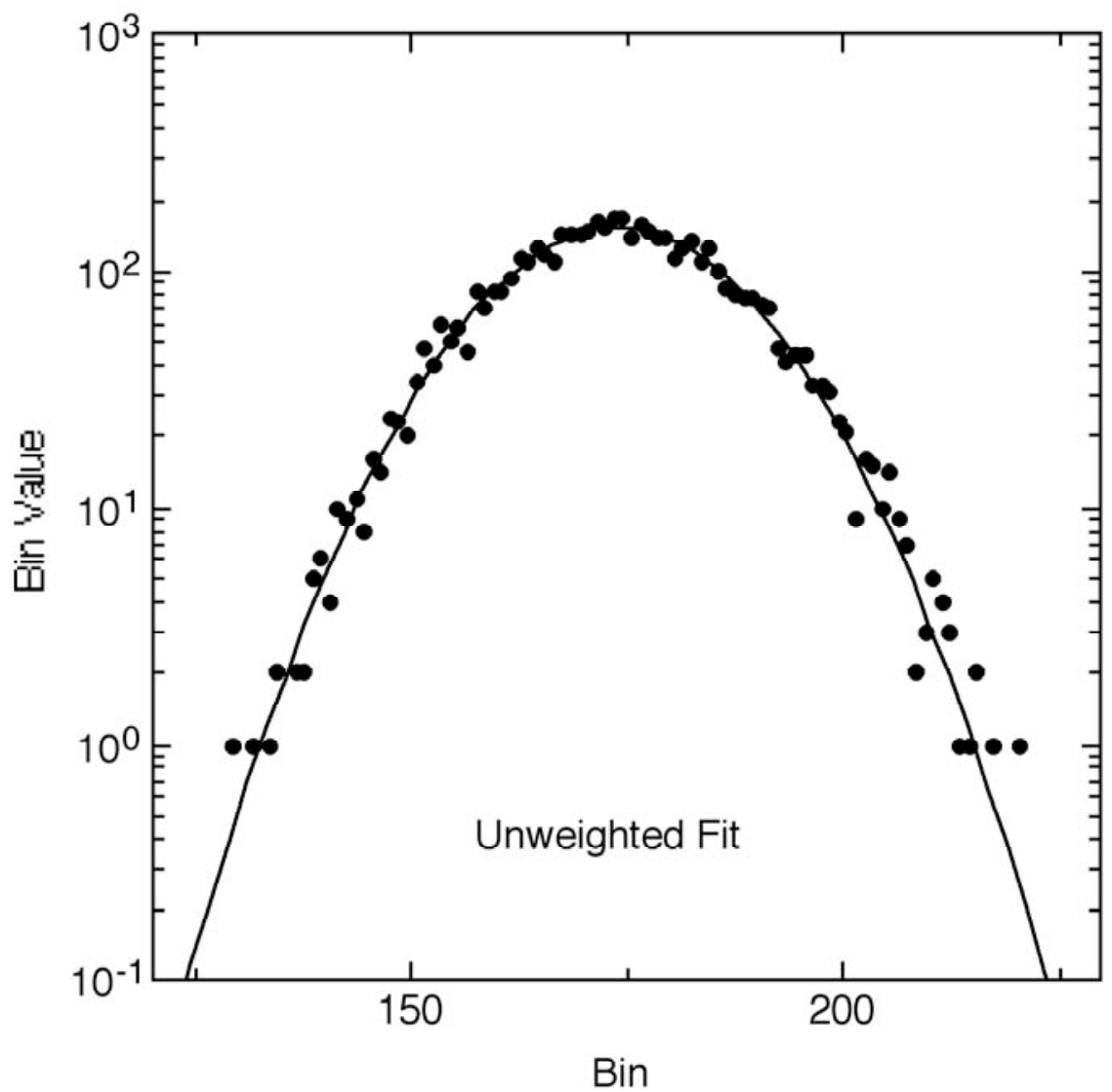

Figure 5.4.3-1 Background variations in a single liquid scintillation detector [35].

The data shown in the figure is the number of counts recorded during a $5 \mathrm{sec}$ observation using a single large liquid scintillation detector, $20 \mathrm{~cm}$ diameter by $183 \mathrm{~cm}$ long. The observation was repeated roughly 50,000 times over several days to acquire the data shown[35]. The lower energy deposition discriminator was set to $\mathrm{E}_{\mathrm{dep}}>3 \mathrm{MeV}$. The mean count rate was $\sim 35 \mathrm{c} / \mathrm{s}$ resulting in $\sim 175$ counts per bin. The solid curve is a Poisson distribution fitted to the data. Examination of the figure shows that the empirical data is well represented by the Poisson distribution out to $\sim 3.5 \square$ to either side of the mean rate. This is important since the principal characteristic of the Poisson distribution is that its variance is equal to the mean, i.e. $\left.\square_{p}^{2}=<\mathrm{C}\right\rangle$.

As discussed earlier we don't know whether the Poisson statistics provide an adequate description of our counting distribution further than $3.5 \square$ from the mean. This will require more exhaustive measurements. However, it is clear that cosmic ray showers do not produce variations from the Poisson distribution in the regime investigated thus far, i.e. in data sampled in $5 \mathrm{sec}$ time bins. We also don't know the extent to which movement of cargo and/or shielding produces larger background variations. For the present time our analyses are based on the Gaussian distribution of count rates, and for the present, we will take the variance to be equal to the mean as is the case for a Poisson distribution. Later measurements in the cargo-scanning lab 
will determine whether our estimate of the variance must be increased for purposes of predicting error rates. It is reasonable to expect that the variance will be much larger when significant masses of cargo are moved about since:

- they are spallation neutron sources for cosmic muons

- they provide shielding for some of the background radiation, though this should be invisible when the system energy threshold is set to or above $3 \mathrm{MeV}$

- $\quad$ and there may be activation products and/or other interferences in the cargo.

Only empirical measurements with a very large number of cargos can establish the variance and whether the statistics are adequately described by the Gaussian distribution. Pending that experience we will use the Gaussian to predict error rates.

\subsection{What dose might be required for reliable scanning?}

The ROC curves in the preceding sections determine the level of detected signal radiation that will be needed to meet the overall system requirements. Those are taken to be $P_{d} \geq 95 \%$ and $\mathrm{P}_{\mathrm{fp}}{ }^{\prime \prime} \leq 0.001$ in this case. The previous section established that sufficient neutron fluence at the SNM target must be produced so that the signal detection rate $S \geq 5 \square_{\square}$.

For the case where no materials or shields are being moved the Poisson variance could be assumed, i.e. $\square_{B}{ }^{2}=<C_{B}>$. For our current detector array $\left\langle\mathrm{C}_{B}>\sim 700 \mathrm{c} / \mathrm{s}\right.$ so that $\mathrm{S}_{\min } \sim 130 \mathrm{c} / \mathrm{s}$. The data could be accumulated for as long as one minute as a container scans down the array so that the integrated counts correspond to $\left\langle\mathrm{C}_{\mathrm{B}}>\sim 4.2 \times 10^{4}, \mathrm{~S}_{\min } \sim 1 \times 10^{3}\right.$ counts.

Empirical data obtained thus far with the $14 \mathrm{MeV}$ neutron source shows the count rate in a single liquid scintillator cell $\sim 100 \mathrm{c} / \mathrm{s}$ with only $380 \mathrm{~g}{ }^{235} \mathrm{U}$ in the target and $40 \mathrm{~g} / \mathrm{cm}^{2}$ wood cargo shielding following a $30 \mathrm{~s}$ irradiation. Integrated counts in the data shown is on the order of $3 \times 10^{3}$. In this case the dose rate at the container wall is $\sim 20 \mathrm{mR} / \mathrm{s}$ for an integrated neutron dose $600 \mathrm{mR}$. Projecting to a realistic cargo scan the goal quantity of $5 \mathrm{~kg}$ would allow this dose to be reduced to $\sim 20 \mathrm{mR}$ assuming the fission rate scales as the surface area of a solid sphere (a factor of $\sim 6 \mathrm{X}$ ), the count rate scales as the detector volume (20X) and the $\mathrm{S}_{\min }$ scales as the square root of the detector volume (4.5X). For thicker shielding the dose would have to be increased to accommodate neutron attenuation in the interrogating beam. This is to be compared to the current guidance on dose rates from the NCRP[36]. There the guidance for neutron based screening systems is a dose to the cargo less than $100 \mathrm{mR}$ per scan for routine screening and less than $500 \mathrm{mR}$ per scan if a national security situation is declared. While these estimates are suggestive of potentially very good detection performance at moderate dose to the cargo, only realistic simulations can determine the actual dose to be expected.

In the real case with materials being moved around may produce a variance larger than for the Poisson distribution. It could be very much larger. Also, the details of neutron attenuation reaching the SNM target and subsequent attenuation of the signature g-radiation are not yet known in detail. A program of validated simulations and experimental measurements will be undertaken to determine these attenuation factors. Then with reliable knowledge of the 
background variance and attenuation factors the ROC curves above can be used to determine the needed signal strength from which the beam intensity requirement will be established. Given the beam intensity a dose estimate for cargo exposure is then readily generated.

\subsection{Activation of cargo components}

Activation of cargo contents will be an important issue of concern as an engineering prototype is designed for field evaluation. Detailed Monte Carlo simulations with reliable databases for the activation cross sections will be required to provide a proper estimate of cargo activity and its decay time following irradiation. That is an essential component of the development program as it goes forward.

Nevertheless, rough estimates using activation cross sections at $\mathrm{E}_{\mathrm{n}}=14 \mathrm{MeV}$ and neglecting the benefits of attenuation have been carried out and are reported in detail[5]. Overall, those rough estimates indicate that brief cooling periods, a few hours or so, result in sufficient decay of activation products that the cargo contents are below the DOT threshold for radioactive designation of the cargo materials. 


\section{Conclusions and summary}

A new signature for SNM detection has been identified and developed. Experimental tests in various lab and cargo simulation environments show this signature to be very robust even when the SNM is well shielded. A new simulation code has been developed to provide the capability to optimize the design of a cargo scanning system based on this signature with neutron interrogation. One major interference has been identified and eliminated by the choice of a different neutron source. Other interferences seem to be of secondary importance at this point but details have not yet been studied. Rough estimates of error rates have been made for the simplest Poisson-distributed background rate and those point to reliable detection of SNM that meets the goal requirements at a source dose well within regulatory requirements so far as they're know today.

\subsection{Brief summary of accomplishments}

- Identified and developed a new signature for SNM detection in thick cargo

- Defined a principal concept for implementation of this signature in a detection system.

- Assembled component testing lab for characterization of proposed system components.

- Developed a new capability for validated simulations to predict the performance of the concept with many variations in the details.

- Made absolute intensity measurements of the new signature and used those to accurately validate simulation tools.

- Defined an appropriate neutron source and detector array.

- Installed the appropriate neutron source and detector array in the cargo-scanning lab.

- Verified simulation tools with experiments employing representative cargo materials at challenging thickness in realistic geometry.

- Used a simplified error prediction analysis to show that goals can be reached within parameters currently under study in the cargo-scanning lab.

\subsection{Remaining issues}

- Background variations can lead to false positive errors. As cargo materials are moved and scanned the background variations are likely to be larger than those for a stable environment. Those variations must be characterized for a realistic environment.

- Component instability due to temperature variations, changes in environment, movement of materials, or other causes can lead to false alarms. These effects need to be quantified. 
- Activation of cargo materials can lead to interferences and false positives. Further studies of a wide variety of cargo materials irradiated under the conditions available in the cargo scanning lab are needed to identify and characterize interferences and their impact on the reliability of this SNM detection technique.

\subsection{Status of the project schedule}

Table 6.3-1 below shows the scheduled tasks together with their completion dates.

Table 6.3-1 Milestone schedule

\begin{tabular}{|l|l|l|l|}
\hline Task & $\begin{array}{l}\text { Scheduled } \\
\text { completion }\end{array}$ & $\begin{array}{l}\text { Actual } \\
\text { completion }\end{array}$ & Reschedule \\
\hline Commission B231 cargo scanning lab & $7 / 27 / 04$ & $8 / 30 / 04$ & \\
\hline Procure 4 MeV RFQ for neutron generator & $9 / 15 / 04$ & $9 / 15 / 04$ & \\
\hline Fabricate 4-element scintillation detector array & $11 / 8 / 04$ & $9 / 30 / 04$ & \\
\hline Construct 3 palletized cargo models in simulations & $1 / 31 / 05$ & & $8 / 15 / 05$ \\
\hline Report interferences and simulation predictions & $3 / 28 / 05$ & $4 / 10 / 05$ & \\
\hline Complete 14 MeV demonstration experiments & $4 / 25 / 05$ & $5 / 12 / 05$ & \\
\hline $\begin{array}{l}\text { Construct 5 heterogeneous cargo models for } \\
\text { simulation }\end{array}$ & $5 / 27 / 05$ & & $8 / 30 / 05$ \\
\hline Complete accelerator pit modifications & $6 / 3 / 05$ & $6 / 15 / 05$ & \\
\hline Commission high output RGD & $6 / 30 / 05$ & & $8 / 20 / 05$ \\
\hline Issue project summary report & $8 / 5 / 05$ & & \\
\hline $\begin{array}{l}\text { Complete initial demonstration experiments at 7 } \\
\text { MeV }\end{array}$ & $10 / 17 / 05$ & & \\
\hline Issue interference report & $10 / 28 / 05$ & & \\
\hline $\begin{array}{l}\text { Complete field prototype system model for } \\
\text { simulations }\end{array}$ & $12 / 15 / 05$ & & \\
\hline
\end{tabular}




\section{References}

1. C. E. Grassley, M. Baucus, B. Thomas, C. B. Rangel, "Container security: Expansion of key customs programs will require greater attention to critical success factors ", General Accounting Office, US Congress, GAO 03-770, July 25, 2003.

2. Maritime_Transport_Committee, "Security in maritime transport: Risk factors and economic impact ", Organization for Economic Co-operation and Development (OECD); Directorate for Science, Technology and Industry, July, 2003.

3. W. Strauch, "Container Handbook, Cargo loss prevention information from German marine insurers", 2005, website, 1 August, http://www.containerhandbuch.de/chb e/stra/index.html.

4. S. Tarver, "PIERS: Global Intelligence Solutions", 2005, PIERS Power Search, website, 1 August, http://www.piers.com/.

5. D. Slaughter, M. Accatino, A. Bernstein, J. Candy, A. Dougan, J. Hall, A. Loshak, D. Manatt, A. Meyer, B. Pohl, S. Prussin, R. Walling, D. Weirup, "Detection of special nuclear material in cargo containers using neutron interrogation ", Lawrence Livermore National Laboratory, UCRL-ID 155315, August, 2003.

6. E. B. Norman, S. G. Prussin, R.-M. Larimer, H. Shugart, E. Browne, A. R. Smith, R. J. McDonald, H. Nitsche, P. Gupta, M. I. Frank, T. B. Gosnell, "Signatures of special nuclear material: High-energy Grays following fission", Nuclear Instruments \& Methods in Physics Research A 521/2, 608 (2004).

7. D. R. Slaughter, M. R. Accatino, A. Bernstein, A. D. Dougan, J. M. Hall, A. Loshak, D. R. Manatt, B. A. Pohl, S. G. Prussin, R. S. Walling, D. L. Weirup, "The "nuclear car wash": a scanner to detect illicit special nuclear material in cargo containers", IEEE Sensors Journal $\mathbf{5}$, 560-564 (2005).

8. D. R. Slaughter, A. Bernstein, J. A. Church, M. A. Descalle, et al., "CE-2: Early results utilizing high-energy fission product $\square$ rays to detect fissionable material in cargo", Conference on Accelerator Applications in Research and Industry, 11-15 October, 2004, Ft. Worth, TX,

9. D. Slaughter, D. Dietrich, "Active neutron scanning intermodal cargo containers to detect well shielded high-enriched uranium and plutonium", Working together: R\&D Partnerships in Homeland Security, April 27-28, 2005, Boston, MA, IEEE.

10. J. M. Hall, J. A. Pruet, D. A. Brown, M.-A. Descalle, G. W. Hedstrom, S. G. Prussin, "Modeling the production of beta-delayed gamma rays for the detection of special nuclear materials ", LLNL, UCRL-TR 209738, 14 February, 2005. 
11. J. Pruet, M.-A. Descalle, J. Hall, B. Pohl, S. G. Prussin, "Neutron and photon transport in seagoing cargo containers", Journal of Applied Physics 97, 94908-1-10 (2005).

12. J. Pruet, J. Hall, M.-A. Descalle, S. Prussin, "Monte Carlo models for the production of delayed gamma-rays following fission of special nuclear materials", Nuclear Instruments and Methods in Physics Research B 222, 403-410 (2005).

13. D. Brown, J. Pruet, G. Hedstrom, J. Hall, " LLNL, UCRL-TR 20660, 2004.

14. LLNL, "A New, High-Resolution Code for Modeling Radiation Transport", 2005, 27 June, http://www-pat.llnl.gov/N_Div/COG/.

15. J. H. Hall, M.-A. Descalle, "Geometry ", LLNL, NCW-Technical Note NCW040604JH, 4 June, 2004.

16. D. Slaughter, D. Rueppel, "Calibration of depangher long counter from $2 \mathrm{keV}$ to 19 MeV", Nuclear Instruments and Methods $\underline{145}$, 315-320 (1977).

17. Saint-Gobain, "Scintillation Products", 2005, 27 June, http://www.detectors.saintgobain.com/.

18. A. Loshak, "Long counter calibration and neutron generator calibration ", LLNL, NCWTechnical Note NCW040910AL, 10 September, 2004.

19. A. Loshak, "Neutron generator calibration ", LLNL, NCW-Technical Note NCW040910aAl, 10 September, 2004.

20. M.-A. Descalle, "First COG Results, Thermal/fast fluences ", LLNL, NCW-Technical Note NCW040915MD, 15 September, 2004.

21. A. Loshak, "Typical scintillation monitor data ", LLNL, NCW Technical Note NCW050201AL, February, 2005.

22. T. R. England, B. F. Rider, "Evaluation and compilation of fission yields ", Los Alamos National Laboratory, ENDF-349 LA-UR 94-3106, October, 1994.

23. J. Hall, "Summary of recent COG simulations I ", LLNL, NCW Technical Note NCW041201JH, December, 2004.

24. K. Raschke, "Calibration of ORTEC 80\% HPGe detector", September, 2004, Personal communication.

25. M.-A. Descalle, "Calibration curve memo ", LLNL, NCW Technical Note NCW041101MD, November, 2004. 
26. M.-A. Descalle, "Simulations of full energy peak deposition in scintillation detectors ", LLNL, NCW-Technical Note NCW041101MD, 11 November, 2004.

27. E. Norman, "Efficiency of HPGe spectrometer ", LLNL, NCW Technical Note NCW050201EN, February, 2005.

28. E. Norman, "Comment on HPGe efficiency ", LLNL, NCW Technical Note NCW050202EN, February, 2005.

29. D. Manatt, "Transmission of ${ }^{60}$ Co radiation through tantalum ", LLNL, NCW-Technical Note NCW050203DM, February, 2005.

30. M.-A. Descalle, "Monte Carlo simulations of HPGe efficiency ", LLNL, NCW Technical Note NCW050201MD, February, 2005.

31. M.-A. Descalle, "Co60 Ge efficiency data ", LLNL, NCW Technical Note NCW050301MD, March, 2005.

32. D. Manatt, "Background beam bug data with no cargo ", LLNL, NCW-Technical Note NCW050302DM, March, 2005.

33. LBNL_Nuclear_Science_Division, "Table of Isotopes", 2003, Lawrence Berkeley National Laboratory, website, http://ie.lbl.gov/toi/.

34. M.-A. Descalle, "Memo on fluences and fission rates in irradiation of bare HEU target with 14-MeV neutrons ", LLNL, NCW Technical Note NCW050301bMD, March, 2005.

35. M. McDowell, "Background count rate distribution ", LLNL, NCW Technical Note NCW040607bMM, 7 June, 2004.

36. NCRP, "Recent applications of the NCRP public dose limit recommendation for ionizing radiation ", National Council on Radiation Protection and Measurements, NCRP Statement No. 10 December, 2004. 